\title{
ON THE PROCONGRUENCE COMPLETION OF THE TEICHMÜLLER MODULAR GROUP
}

\begin{abstract}
MARCO BOGGI
Abstract. For $2 g-2+n>0$, the Teichmüller modular group $\Gamma_{g, n}$ of a compact Riemann surface of genus $g$ with $n$ points removed, $S_{g, n}$ is the group of homotopy classes of diffeomorphisms of $S_{g, n}$ which preserve the orientation of $S_{g, n}$ and a given order of its punctures. Let $\Pi_{g, n}$ be the fundamental group of $S_{g, n}$, with a given base point, and $\hat{\Pi}_{g, n}$ its profinite completion. There is then a natural faithful representation $\Gamma_{g, n} \hookrightarrow \operatorname{Out}\left(\hat{\Pi}_{g, n}\right)$. The procongruence Teichmüller group $\check{\Gamma}_{g, n}$ is defined to be the closure of the Teichmüller group $\Gamma_{g, n}$ inside the profinite group $\operatorname{Out}\left(\hat{\Pi}_{g, n}\right)$.

In this paper, we begin a systematic study of the procongruence completion $\check{\Gamma}_{g, n}$. The set of profinite Dehn twists of $\check{\Gamma}_{g, n}$ is the closure, inside this group, of the set of Dehn twists of $\Gamma_{g, n}$. The main technical result of the paper is a parametrization of the set of profinite Dehn twists of $\check{\Gamma}_{g, n}$ and the subsequent description of their centralizers (Sections 5 and 6). This is the basis for the Grothendieck-Teichmüller Lego with procongruence Teichmüller groups as building blocks.

As an application, in Section 7, we prove that some Galois representations associated to hyperbolic curves over number fields and their moduli spaces are faithful.
\end{abstract}

\section{INTRODUCTION}

Let $C$ be a hyperbolic curve defined over a number field $\mathbb{k}$. Let us fix an embedding $\mathbb{k} \subset \overline{\mathbb{Q}}$ and a $\overline{\mathbb{Q}}$-valued point $\tilde{\xi} \in C$. Then, the structural morphism $C \rightarrow \operatorname{Spec}(\mathbb{k})$ induces a short exact sequence of algebraic fundamental groups,

$$
1 \rightarrow \pi_{1}\left(C \times_{\mathbb{k}} \overline{\mathbb{Q}}, \tilde{\xi}\right) \rightarrow \pi_{1}(C, \tilde{\xi}) \rightarrow G_{\mathbb{k}} \rightarrow 1,
$$

where the absolute Galois group $G_{\mathbb{k}}$ is identified with the algebraic fundamental group $\pi_{1}(\operatorname{Spec}(\mathbb{k}), \operatorname{Spec}(\overline{\mathbb{Q}}))$. Associated to the above short exact sequence is the outer Galois representation:

$$
\rho_{C}: G_{\mathbb{k}} \rightarrow \operatorname{Out}\left(\pi_{1}\left(C \times_{\mathbb{k}} \overline{\mathbb{Q}}, \tilde{\xi}\right)\right) .
$$

This representation plays a central role in the arithmetic geometry of curves.

The representation $\rho_{C}$ can be interpreted as the monodromy representation associated to the curve $C \rightarrow \operatorname{Spec}(\mathbb{k})$ and the given choices of base points. Hence, it can be recovered from the arithmetic universal monodromy representation as follows.

Let us suppose that $C$ is an $n$-punctured, genus $g$ curve, for $2 g-2+n>0$. Then let $\mathcal{M}_{g, n}$ be the moduli stack of smooth $n$-pointed, genus $g$ curves defined over some number field. It is a smooth irreducible Deligne-Mumford stack of dimension $3 g-3+n$ defined over $\operatorname{Spec}(\mathbb{Q})$, endowed with a universal $n$-punctured, genus $g$

Received by the editors November 9, 2011 and, in revised form, September 3, 2012.

2010 Mathematics Subject Classification. Primary 14H10, 30F60, 11F80, 14H30, 14 F35. 
curve $\mathscr{C} \rightarrow \mathcal{M}_{g, n}$. Let $\xi \in \mathcal{M}_{g, n}$ be the point corresponding to the curve $C$ and $\bar{\xi}$ the $\overline{\mathbb{Q}}$-valued point lying over it. Let us identify the fiber $\mathscr{C}_{\bar{\xi}}$ with the curve $C \times_{\mathbb{k}} \overline{\mathbb{Q}}$. There is then a short exact sequence of algebraic fundamental groups:

$$
1 \rightarrow \pi_{1}\left(C \times_{\mathbb{k}} \overline{\mathbb{Q}}, \tilde{\xi}\right) \rightarrow \pi_{1}(\mathscr{C}, \tilde{\xi}) \rightarrow \pi_{1}\left(\mathcal{M}_{g, n}, \bar{\xi}\right) \rightarrow 1 .
$$

The associated outer representation

$$
\mu_{g, n}: \pi_{1}\left(\mathcal{M}_{g, n}, \bar{\xi}\right) \rightarrow \operatorname{Out}\left(\pi_{1}\left(C \times_{\mathbb{k}} \overline{\mathbb{Q}}, \tilde{\xi}\right)\right)
$$

is the arithmetic universal monodromy representation.

The morphism of pointed stacks $\xi:(\operatorname{Spec}(\mathbb{k}), \operatorname{Spec}(\overline{\mathbb{Q}})) \rightarrow\left(\mathcal{M}_{g, n}, \bar{\xi}\right)$ induces a homomorphism $\xi_{*}: G_{\mathbb{k}} \rightarrow \pi_{1}\left(\mathcal{M}_{g, n}, \bar{\xi}\right)$ on algebraic fundamental groups. The representation $\rho_{C}$ is then obtained by composing $\xi_{*}$ with the arithmetic universal monodromy representation $\mu_{g, n}$. Thus, it is not a surprise that the representation $\mu_{g, n}$ contains essential information on the properties shared by all Galois outer representations associated to smooth $n$-punctured, genus $g$ arithmetic curves. The study of the representation $\mu_{g, n}$ is one of the principal purposes of this paper.

The structural morphism $\mathcal{M}_{g, n} \rightarrow \operatorname{Spec}(\mathbb{Q})$ induces the short exact sequence:

$$
1 \rightarrow \pi_{1}\left(\mathcal{M}_{g, n} \times \overline{\mathbb{Q}}, \bar{\xi}\right) \rightarrow \pi_{1}\left(\mathcal{M}_{g, n}, \bar{\xi}\right) \rightarrow G_{\mathbb{Q}} \rightarrow 1
$$

The left term of this short exact sequence is called the geometric algebraic fundamental group of $\mathcal{M}_{g, n}$. The reason for this terminology is that it is naturally isomorphic to the profinite completion of the topological fundamental group with base point $\bar{\xi}$ of the complex analytic stack associated to the complex Deligne-Mumford stack $\mathcal{M}_{g, n} \times \mathbb{C}$ (cf. [18, [19]).

Let $S_{g, n}$ be an $n$-punctured, genus $g$ Riemann surface and let $\Gamma_{g, n}$ be the corresponding pure Teichmüller modular group. The choice of a homeomorphism $\phi: S_{g, n} \rightarrow\left(C \times_{\mathbb{k}} \mathbb{C}\right)^{\text {top }}$ determines an isomorphism between the group $\Gamma_{g, n}$ and the topological fundamental group $\pi_{1}^{\text {top }}\left(\mathcal{M}_{g, n} \times \mathbb{C}, \bar{\xi}\right)$ (for more details, see Section 2) so that we also get an identification of the geometric algebraic fundamental group of $\mathcal{M}_{g, n}$ with the profinite completion of the Teichmüller group $\Gamma_{g, n}$, which we simply call the profinite Teichmüller group $\hat{\Gamma}_{g, n}$.

Let us denote by $\Pi_{g, n}$ the topological fundamental group $\pi_{1}\left(S_{g, n}, \phi^{-1}(\tilde{\xi})\right)$ and by $\hat{\Pi}_{g, n}$ its profinite completion. The restriction of the arithmetic universal monodromy representation $\mu_{g, n}$ to the geometric algebraic fundamental group then induces a representation,

$$
\hat{\rho}_{g, n}: \hat{\Gamma}_{g, n} \rightarrow \operatorname{Out}\left(\hat{\Pi}_{g, n}\right),
$$

which we call the profinite universal monodromy representation. This can also be described as the homomorphism of profinite groups induced by the canonical homotopy action of $\Gamma_{g, n}$ on $S_{g, n}$ (see Section 2).

It is clear that, in order to gain some insight on the representation $\mu_{g, n}$, we need to first study the representation $\hat{\rho}_{g, n}$. The study of the latter representation is the main subject of the paper from Section 2 until Section 6 .

The first question which arises about the profinite universal monodromy representation $\hat{\rho}_{g, n}$ is whether or not it is faithful. This is better known as the congruence subgroup problem for the Teichmüller group $\Gamma_{g, n}$. An affirmative answer is known only for genus $\leq 2$ (cf. [3], [8]).

The investigation underlying this paper was stimulated by the idea that, in order to advance in all the above issues, it was necessary to have a complete understanding 
of the group-theoretic properties of the image of the representation $\hat{\rho}_{g, n}$, which we denote by $\check{\Gamma}_{g, n}$ and call the procongruence Teichmüller group.

The combinatorial group theory of the Teichmüller group $\Gamma_{g, n}$ begins with the study of the relations occurring between words in its standard set of generators which are the Dehn twists. The set of profinite Dehn twists of the procongruence Teichmüller group $\check{\Gamma}_{g, n}$ is the closure of the image of the set of Dehn twists via the natural monomorphism $\Gamma_{g, n} \hookrightarrow \check{\Gamma}_{g, n}$.

The key technical result of this paper is the characterization of powers of profinite twists in $\check{\Gamma}_{g, n}$ which is given in Theorem 5.1. As an immediate consequence, we get a description of the centralizers and of the normalizers of the closed abelian subgroups spanned by the set of powers of profinite twists in $\check{\Gamma}_{g, n}$, in perfect analogy with the classical results in $\Gamma_{g, n}$ (see Corollary 6.4 and Theorem 6.6).

Deeper and more involved relations occurring between Dehn twists in $\Gamma_{g, n}$ are encoded in the various curve complexes which can be associated to $S_{g, n}$. Here, we mention just the most important one: the complex of curves $C\left(S_{g, n}\right)$. This is the simplicial complex whose simplices are given by sets of distinct, non-trivial, isotopy classes of simple closed curves (briefly s.c.c.) on $S_{g, n}$ such that they admit a set of disjoint representatives, none of them bounding a disc with a single puncture. This complex is endowed with a natural simplicial action of the Teichmüller group $\Gamma_{g, n}$.

Sections 3 and 4 are devoted to constructing a satisfactory profinite analogue of the curve complex $C\left(S_{g, n}\right)$. As the final result, we get a simplicial profinite complex $L\left(\hat{\Pi}_{g, n}\right)$ which we call the complex of profinite curves. Eventually, in Theorem 6.9. this is characterized as the simplicial complex whose $k$-simplices are the closed abelian subgroups of rank $k+1$ spanned by profinite Dehn twists in $\check{\Gamma}_{g, n}$.

The importance of the complex of profinite curves is well illustrated by Corollary 7.2 of [9], where it is shown that the obstruction for the congruence subgroup property to hold in genus greater than 2 is the profinite fundamental group of $L\left(\hat{\Pi}_{g}\right)$, for $g>2$.

All the results mentioned above then form the basis for the study of Galois representations associated to hyperbolic curves carried out in Section 7 The main result is that the outer representation $\rho_{C}$ associated to a hyperbolic curve $C$ over a number field $\mathbb{k}$ is faithful (Theorem 7.7). The faithfulness of the arithmetic universal monodromy representation $\mu_{g, n}$ is instead reduced to the congruence subgroup problem for $\Gamma_{g, n}$. In particular, it follows that $\mu_{g, n}$ is faithful for $g \leq 2$ (Corollary (7.10). The proofs of all these statements, especially Theorem 7.4, are inspired by the ideas of Grothendieck-Teichmüller theory as they appeared in the Esquisse d'un Programme [13. With a substantially different approach, similar results have been proved in [17] and [15].

\section{LEVEL STRUCTURES OVER MOdULI OF CURVES}

The study we are going to carry out from Section 2 to Section 6 is in essence topological. Hence, in order to avoid cumbersome notation, for a complex DeligneMumford (briefly $\mathrm{D}-\mathrm{M}$ ) stack $X$, we will denote by $\pi_{1}(X)$ its topological fundamental group and by $\hat{\pi}_{1}(X)$ its algebraic fundamental group (when unnecessary, we omit mentioning the base points). This notation is consistent with the fact that $\hat{\pi}_{1}(X)$ is isomorphic to the profinite completion of $\pi_{1}(X)$. For the same reasons, $\overline{\mathcal{M}}_{g, n}$, for $2 g-2+n>0$, will denote the stack of $n$-pointed, genus $g$, stable complex algebraic curves. It is a smooth irreducible proper complex D-M stack of dimension 
$3 g-3+n$, and it contains, as an open substack, the stack $\mathcal{M}_{g, n}$ of $n$-pointed, genus $g$, smooth complex algebraic curves. We will keep the same notation to denote the respective underlying analytic and topological stacks.

The stack $\overline{\mathcal{M}}_{g, n}$ is simply connected. On the contrary, the stack $\mathcal{M}_{g, n}$ has plenty of non-trivial coverings which we are briefly going to introduce in this section. We refer to 9 for more details and references on all the following constructions.

The universal cover of $\mathcal{M}_{g, n}$ is the Teichmüller space $T_{g, n}$. This is the stack of $n$-pointed, genus $g$, smooth complex analytic curves $\left(\mathscr{E} \rightarrow \mathscr{U}, s_{1}, \ldots, s_{n}\right)$ endowed with a topological trivialization $\Phi: S_{g, n} \times \mathscr{U} \stackrel{\sim}{\rightarrow} \mathscr{E} \backslash \bigcup_{i=1}^{n} s_{i}(\mathscr{U})$ over $\mathscr{U}$, where two such trivializations are considered equivalent when they are homotopic over $\mathscr{U}$. We then denote the corresponding object of $T_{g, n}$ by $\left(\mathscr{E} \rightarrow \mathscr{U}, s_{1}, \ldots, s_{n}, \Phi\right)$ or, when $\mathscr{U}$ is just one point, simply by $(E, \Phi)$. The complex analytic stack $T_{g, n}$ is represented by a contractible complex manifold. Then, the natural map of complex analytic stacks $T_{g, n} \rightarrow \mathcal{M}_{g, n}$ is a universal cover. The deck transformation group of this covering is described as follows.

Let $\mathrm{Hom}^{+}\left(S_{g, n}\right)$ be the group of orientation preserving self-homeomorphisms of $S_{g, n}$ and $\operatorname{Hom}^{0}\left(S_{g, n}\right)$ be the subgroup consisting of homeomorphisms homotopic to the identity. The mapping class group or Teichmüller modular group $\Gamma_{g,[n]}$ is classically defined to be the group of homotopy classes of homeomorphisms of $S_{g, n}$ which preserve the orientation,

$$
\Gamma_{g,[n]}:=\operatorname{Hom}^{+}\left(S_{g, n}\right) / \operatorname{Hom}^{0}\left(S_{g, n}\right),
$$

where $\operatorname{Hom}_{0}\left(S_{g, n}\right)$ is the connected component of the identity in the topological group of homeomorphisms $\operatorname{Hom}^{+}\left(S_{g, n}\right)$. This group is then the group of deck transformation of the étale covering $T_{g, n} \rightarrow \mathcal{M}_{g,[n]}$, where the latter denotes the stack of genus $g$, smooth complex curves with $n$ unordered markings. Therefore, there is a short exact sequence,

$$
1 \rightarrow \Gamma_{g, n} \rightarrow \Gamma_{g,[n]} \rightarrow \Sigma_{n} \rightarrow 1,
$$

where $\Sigma_{n}$ denotes the symmetric group on the set of punctures of $S_{g, n}$ and $\Gamma_{g, n}$ is the pure mapping class group or pure Teichmüller modular group associated to $S_{g, n}$ and the deck transformation group of the covering $T_{g, n} \rightarrow \mathcal{M}_{g, n}$.

There is a natural way to define homotopy groups for topological D-M stacks (cf. [19]) so that the choice of a point $a=[C] \in \mathcal{M}_{g, n}$ and a homeomorphism $\phi: S_{g, n} \rightarrow$ $C$ identifies the topological fundamental groups $\pi_{1}\left(\mathcal{M}_{g, n}, a\right)$ and $\pi_{1}\left(\mathcal{M}_{g,[n]}, a\right)$ with the pure mapping class group $\Gamma_{g, n}$ and with the mapping class group $\Gamma_{g,[n]}$, respectively.

Let $p: \mathscr{C} \rightarrow \mathcal{M}_{g,[n]}$ be the universal $n$-punctured, genus $g$ curve. Since $p$ is a Serre fibration and $\pi_{2}\left(\mathcal{M}_{g,[n]}\right)=\pi_{2}\left(T_{g, n}\right)=0$, there is a short exact sequence,

$$
1 \rightarrow \pi_{1}\left(\mathscr{C}_{a}, \tilde{a}\right) \rightarrow \pi_{1}(\mathscr{C}, \tilde{a}) \rightarrow \pi_{1}\left(\mathcal{M}_{g,[n]}, a\right) \rightarrow 1,
$$

where $\tilde{a}$ is a point in the fiber $\mathscr{C}_{a}$. By a standard argument this defines a monodromy representation,

$$
\rho_{g,[n]}: \pi_{1}\left(\mathcal{M}_{g,[n]}, a\right) \rightarrow \operatorname{Out}\left(\pi_{1}\left(\mathscr{C}_{a}, \tilde{a}\right)\right)
$$

called the universal monodromy representation. We then denote by $\rho_{g, n}$ its restriction to the pure mapping class group $\Gamma_{g, n}$.

Let us then fix a homeomorphism $\phi: S_{g, n} \rightarrow \mathscr{C}_{a}$ and let $\Pi_{g, n}$ be the fundamental group of $S_{g, n}$ based in $\phi^{-1}(\tilde{a})$. Then, the representation $\rho_{g,[n]}$ is identified with the 
faithful representation $\Gamma_{g,[n]} \hookrightarrow \operatorname{Out}\left(\Pi_{g, n}\right)$, induced by the homotopy action of $\Gamma_{g,[n]}$ on the Riemann surface $S_{g, n}$.

In this paper, a level structure $\mathcal{M}^{\lambda}$ is a finite, connected, Galois, étale covering of the stack $\mathcal{M}_{g,[n]}$ (by an étale covering, here we mean an étale, surjective, representable morphism of algebraic stacks); therefore, it is represented by a smooth $\mathrm{D}-\mathrm{M}$ stack $\mathcal{M}^{\lambda}$ as well. This is in contrast with [6], where the covering was not required to be Galois. The level associated to $\mathcal{M}^{\lambda}$ is the finite index normal subgroup $\Gamma^{\lambda}:=\pi_{1}\left(\mathcal{M}^{\lambda}, a^{\prime}\right)$ of the Teichmüller group $\Gamma_{g,[n]}$.

For a given level $\Gamma^{\lambda}$ of $\Gamma_{g,[n]}$, the intersection $\Gamma^{\lambda} \cap \Gamma_{g, n}$ is also a level of $\Gamma_{g,[n]}$ which we denote by $\Gamma_{g, n}^{\lambda}$. The corresponding level structure is denoted by $\mathcal{M}_{g, n}^{\lambda}$. Equivalently, the level structure $\mathcal{M}_{g, n}^{\lambda}$ is the pull-back over $\mathcal{M}_{g, n} \rightarrow \mathcal{M}_{g,[n]}$ of the level structure $\mathcal{M}^{\lambda}$.

The most natural way to define levels is by means of the universal monodromy representation $\rho_{g,[n]}$. For a characteristic subgroup $K \leq \Pi_{g, n}$, let us define the representation,

$$
\rho_{K}: \Gamma_{g,[n]} \rightarrow \operatorname{Out}\left(\Pi_{g, n} / K\right),
$$

whose kernel we denote by $\Gamma^{K}$. When $K$ has finite index in $\Pi_{g, n}$, then $\Gamma^{K}$ has finite index in $\Gamma_{g,[n]}$ and is called the geometric level associated to $K$. The corresponding level structure is denoted by $\mathcal{M}^{K}$.

Of particular interest are the levels defined by the kernels of the representations:

$$
\rho_{(m)}: \Gamma_{g,[n]} \rightarrow \operatorname{Sp}\left(H_{1}\left(S_{g}, \mathbb{Z} / m\right)\right), \quad \text { for } m \geq 2 .
$$

They are denoted by $\Gamma(m)$ and called abelian levels of order $m$. The corresponding level structures are then denoted by $\mathcal{M}^{(m)}$. A classical result of Serre says that an automorphism of a smooth curve acting trivially on its first homology group with $\mathbb{Z} / m$ coefficients, for $m \geq 3$, is trivial. This implies that any level structure $\mathcal{M}^{\lambda}$ dominating an abelian level structure $\mathcal{M}^{(m)}$, with $m \geq 3$, is representable in the category of algebraic varieties.

Another way to define levels, introduced in 9], will be extremely useful here. Let us briefly recall this construction, referring to [9] for more details.

Let $K$ be a proper normal finite index subgroup of $\Pi_{g, n}$, which is invariant for the action of $\Gamma_{g,[n]}$, and let $p_{K}: S_{K} \rightarrow S_{g, n}$ be the étale Galois covering with deck transformation group $G_{K}$ associated to such a subgroup. Let us denote by $\Gamma\left(S_{K}\right)$ the orientation preserving mapping class group of the Riemann surface $S_{K}$. There are then a natural monomorphism $G_{K} \hookrightarrow \Gamma\left(S_{K}\right)$ and a natural epimorphism from the normalizer $N_{\Gamma\left(S_{K}\right)}\left(G_{K}\right)$ of $G_{K}$ in $\Gamma\left(S_{K}\right)$ to the mapping class group $\Gamma_{g,[n]}$. So, there is a natural short exact sequence:

$$
1 \rightarrow G_{K} \rightarrow N_{\Gamma\left(S_{K}\right)}\left(G_{K}\right) \rightarrow \Gamma_{g,[n]} \rightarrow 1 .
$$

From Hurwitz's Theorem and the fact that $K$ is a proper invariant subgroup of $\Pi_{g, n}$, it follows that, for $g \geq 1$ or $n \geq 4$, the compact Riemann surface $\bar{S}_{K}$ obtained by filling in the punctures of $S_{K}$ has genus at least one. For $m \geq 2$, let us then consider the natural representation $\rho_{(m)}: \Gamma\left(S_{K}\right) \rightarrow \operatorname{Sp}\left(H_{1}\left(\bar{S}_{K}, \mathbb{Z} / m\right)\right)$.

For $m \geq 3$ and $m=0$, the restriction of $\rho_{(m)}$ to $G_{K}$ is faithful. For $m=2$, the restriction $\rho_{(m)} \mid G_{K}$ is faithful unless $G_{K}$ contains a hyperelliptic involution, in which case the hyperelliptic involution generates the kernel of $\left.\rho_{(m)}\right|_{G_{K}}$ (cf. Lemma 2.9, Ch. XVII, 2]). In particular, for $g \geq 1$ or for $g=0$, if $\left[\Pi_{0, n}: K\right]>2$, the group 
$G_{K}$ does not contain a hyperelliptic involution and so the restriction of $\rho_{(2)}$ to $G_{K}$ is faithful as well.

Let us assume that the restriction $\left.\rho_{(m)}\right|_{G_{K}}$ is faithful and let us denote by $G_{K}$ its image. For $m \geq 2$, there is a natural representation:

$$
\rho_{K,(m)}: \Gamma_{g,[n]} \rightarrow N_{\mathrm{Sp}\left(H_{1}\left(\bar{S}_{K}, \mathbb{Z} / m\right)\right)}\left(G_{K}\right) / G_{K} .
$$

Let us denote the kernel of $\rho_{K,(m)}$ by $\Gamma^{K,(m)}$ and call it the Looijenga level associated to the finite index invariant subgroup $K$ of $\Pi_{g, n}$. The corresponding level structure is denoted by $\mathcal{M}^{K,(m)}$.

Geometric levels can also be described in terms of the exact sequence (2.1). The geometric level $\Gamma^{K}$ associated to $K$ is indeed the set of elements of $\Gamma_{g,[n]}$ which admit a lift, by the natural epimorphism $N_{\Gamma\left(S_{K}\right)}\left(G_{K}\right) \rightarrow \Gamma_{g,[n]}$, to the centralizer $Z_{\Gamma\left(S_{K}\right)}\left(G_{K}\right)$. Thus, there is a short exact sequence,

$$
1 \rightarrow Z\left(G_{K}\right) \rightarrow Z_{\Gamma\left(S_{K}\right)}\left(G_{K}\right) \rightarrow \Gamma^{K} \rightarrow 1,
$$

where $Z\left(G_{K}\right)$ is the center of the group $G_{K}$. If this center is trivial, the geometric level $\Gamma^{K}$ is naturally identified with the centralizer of $G_{K}$ inside $\Gamma\left(S_{K}\right)$.

A key fact is that the tower of Loojenga levels is equivalent to the tower of geometric levels. More precisely, it holds (Theorem 2.2 and Corollary 2.3 in [9]):

Theorem 2.1. $\quad$ i) For $2 g-2+n>0$, let $K$ be a finite index invariant subgroup of $\Pi_{g, n}$. Then, for $m \geq 3$, or, if the quotient group $G_{K}$ does not contain a hyperelliptic involution, for $m \geq 2$, there is an inclusion of levels $\Gamma^{K,(m)} \unlhd \Gamma^{K}$.

ii) For any fixed integer $m \geq 2$, the set of Looijenga levels $\left\{\Gamma^{K,(m)}\right\}_{K \unlhd \Pi_{g, n}}$ is an inverse system of finite index normal subgroups of $\Gamma_{g,[n]}$ which defines the same profinite topology as the tower of geometric levels $\left\{\Gamma^{K}\right\}_{K \unlhd \Pi_{g, n}}$.

The usual way to compactify a level structure $\mathcal{M}^{\lambda}$ over $\mathcal{M}_{g,[n]}$ is to take the normalization of $\overline{\mathcal{M}}_{g,[n]}$ in the function field of $\mathcal{M}^{\lambda}$. A more functorial definition can be given in the category of regular log schemes. Indeed, it is easy to see that the natural morphism of logarithmic stacks $\left(\overline{\mathcal{M}}^{\lambda}\right)^{l o g} \rightarrow \overline{\mathcal{M}}_{g,[n]}^{\log }$ is log-étale, where $\left(_{-}\right)^{\log }$ denotes the logarithmic structure associated to the respective D-M boundaries $\partial \mathcal{M}^{\lambda}:=\overline{\mathcal{M}}^{\lambda} \backslash \mathcal{M}^{\lambda}$ and $\partial \mathcal{M}_{g,[n]}:=\overline{\mathcal{M}}_{g,[n]} \backslash \mathcal{M}_{g,[n]}$. Vice versa, by the log purity theorem, any finite, connected, log étale Galois covering of $\overline{\mathcal{M}}_{g,[n]}^{l o g}$ is of the above type.

A basic property of (compactified) level structures, proved by Brylinski and Deligne (cf. (ii), Proposition 2 in [10] and Proposition 3.2 in [9]), is that if the level $\Gamma^{\lambda}$ is contained in an abelian level of order $m$, for some $m \geq 3$, then the level structure $\overline{\mathcal{M}}_{g, n}^{\lambda}$ is represented by a projective variety.

An analogue of the $\mathrm{D}-\mathrm{M}$ compactification $\overline{\mathcal{M}}_{g, n}$ of $\mathcal{M}_{g, n}$, at the level of Teichmüller space, is the Bers bordification $\bar{T}_{g, n}$ of the Teichmüller space $T_{g, n}$, which we are shortly going to describe (see $\S 3$, Ch. II, [1] for more details on this construction).

For a given stable $n$-pointed, genus $g$ curve $C$, let $\mathcal{N}$ be its singular set and $\mathcal{P}$ its set of marked points. A degenerate marking $\phi: S_{g, n} \rightarrow C$ is a continuous map such that $C \backslash \operatorname{Im} \phi=\mathcal{P}$, the inverse image $\phi^{-1}(x)$, for all $x \in \mathcal{N}$, is a simple closed curve (briefly, s.c.c.) on $S_{g, n}$ and the restriction of the marking 
$\phi: S_{g, n} \backslash \phi^{-1}(\mathcal{N}) \rightarrow C \backslash(\mathcal{N} \cup \mathcal{P})$ is a homeomorphism. The Bers bordification $\bar{T}_{g, n}$ of the Teichmüller space $T_{g, n}$ is the real analytic space which parametrizes pairs $(C, \phi)$, consisting of a stable $n$-pointed, genus $g$ curve $C$ and the homotopy class of a degenerate marking $\phi: S_{g, n} \rightarrow C$.

The natural action of $\Gamma_{g,[n]}$ on $T_{g, n}$ extends to $\bar{T}_{g, n}$. However, this action is no longer proper and discontinuous, since a boundary stratum has for its inertia group the free abelian group generated by the Dehn twists along the simple closed curves of $S_{g, n}$ which are collapsed on such a boundary stratum.

The geometric quotient $\bar{T}_{g, n} / \Gamma_{g,[n]}$ identifies with the real-analytic space underlying the coarse moduli space $\bar{M}_{g,[n]}$ of stable $n$-pointed, genus $g$ curves. On the other hand, the quotient stack $\left[\bar{T}_{g, n} / \Gamma_{g,[n]}\right]$ only admits a non-representable natural map to the $\mathrm{D}-\mathrm{M}$ stack $\overline{\mathcal{M}}_{g,[n]}$, because of the extra inertia at infinity.

The irreducible components of the boundary $\partial T_{g, n}:=\bar{T}_{g, n} \backslash T_{g, n}$ are isomorphic either to $\bar{T}_{g-1, n+2}$ or to $\bar{T}_{g_{1}, n_{1}+1} \times \bar{T}_{g_{2}, n_{2}+1}$ for some non-negative integers $n_{1}, n_{2}$ and $g_{1}, g_{2}$ such that $n=n_{1}+n_{2}$ and $g=g_{1}+g_{2}$, with the condition that $n_{i} \geq 2$ when $g_{i}=0$.

The nerve of the cover of the boundary $\partial T_{g, n}$ by its irreducible components is explicitly described by a simplicial complex called the complex of curves and is denoted by $C\left(S_{g, n}\right)$. It is the simplicial complex whose simplices are sets of distinct, non-trivial isotopy classes of s.c.c.'s on $S_{g, n}$, such that they admit a set of disjoint representatives, none of them bounding a disc with a single puncture.

The above description of the nerve of the boundary of $\bar{T}_{g, n}$ can be extended to level structures satisfying some mild hypotheses. For a simplex $\sigma \in C\left(S_{g, n}\right)$, let us denote by $\Gamma_{\sigma}$ the stabilizer of $\sigma$ for the natural action of the Teichmüller group $\Gamma_{g, n}$ on $C\left(S_{g, n}\right)$ and by $\Gamma_{\vec{\sigma}}$ the subgroup of elements of $\Gamma_{\sigma}$ which fix all the s.c.c.'s in the simplex $\sigma$, moreover preserving their orientations. It then holds (cf. Proposition 4.2 in [9]):

Proposition 2.2. For $2 g-2+n>0$, let $\Gamma^{\lambda}$ be a level of $\Gamma_{g, n}$ contained in an abelian level $\Gamma(m)$, for $m \geq 3$. Then, it holds that $\Gamma^{\lambda} \cap \Gamma_{\vec{\sigma}}=\Gamma^{\lambda} \cap \Gamma_{\sigma}$, i.e. the level $\Gamma^{\lambda}$ operates without inversions on the curve complex $C\left(S_{g, n}\right)$.

For the level structures $\overline{\mathcal{M}}^{\lambda}$ such that $\Gamma^{\lambda}$ is contained in an abelian level $\Gamma(m)$, for some $m \geq 3$, there is then a direct way to realize the nerve of the cover of their $\mathrm{D}-\mathrm{M}$ boundary by irreducible components in the category of simplicial sets.

Definition 2.3. Let $C\left(S_{g, n}\right)$. be the simplicial set associated to the simplicial complex $C\left(S_{g, n}\right)$ and an ordering of its vertices compatible with the action of a level $\Gamma^{\lambda}$ contained in an abelian level $\Gamma(m)$, for some $m \geq 3$. Then, the simplicial finite set $C^{\lambda}\left(S_{g, n}\right) \bullet$ is defined to be the quotient of $C\left(S_{g, n}\right)$ • by the simplicial action of $\Gamma^{\lambda}$. This simplicial set describes the nerve of the cover of $\partial \mathcal{M}^{\lambda}$ by its irreducible components.

\section{Profinite Completions}

For $2 g-2+n>0$, there is a short exact sequence:

$$
1 \rightarrow \hat{\Pi}_{g, n} \rightarrow \hat{\Gamma}_{g, n+1} \rightarrow \hat{\Gamma}_{g, n} \rightarrow 1 .
$$


The action induced by the restriction of inner automorphisms of $\hat{\Gamma}_{g, n+1}$ to its normal subgroup $\hat{\Pi}_{g, n}$ defines a representation $\tilde{\rho}_{g, n}: \hat{\Gamma}_{g, n+1} \rightarrow \operatorname{Aut}\left(\hat{\Pi}_{g, n}\right)$, which then induces the profinite universal monodromy representation $\hat{\rho}_{g, n}: \hat{\Gamma}_{g, n} \rightarrow \operatorname{Out}\left(\hat{\Pi}_{g, n}\right)$.

Definition 3.1. For $2 g-2+n>0$, let us define the profinite group $\check{\Gamma}_{g, n}$ to be the image of $\hat{\rho}_{g, n}$ in Out $\left(\hat{\Pi}_{g, n}\right)$. We then call $\check{\Gamma}_{g, n}$ the procongruence completion of the Teichmüller group or, more simply, the procongruence Teichmüller group.

By definition, there is a natural continuous homomorphism $i: \Gamma_{g, n} \rightarrow \check{\Gamma}_{g, n}$ with dense image. That this map is also injective is a deeper result by Grossman 12 .

By Theorem 2.4 in [8], for $2 g-2+n>0$ there is a natural isomorphism of profinite groups $\operatorname{Im} \hat{\rho}_{g, n+1} \equiv \operatorname{Im} \tilde{\rho}_{g, n+1}$ and then a natural short exact sequence:

$$
1 \rightarrow \hat{\Pi}_{g, n} \rightarrow \check{\Gamma}_{g, n+1} \rightarrow \check{\Gamma}_{g, n} \rightarrow 1 .
$$

We will now associate to the procongruence completion of the Teichmüller group introduced above a completion of the curve complex. This, however, requires a preliminary digression. Let us recall some definitions from [6].

Let $X_{\bullet}$ be a simplicial set and $G$ a group. A geometric action of $G$ on $X_{\bullet}$ is an action of $G$ on $X_{n}$, defined for all $n \geq 0$, satisfying the following conditions:

i) For all $g \in G$ and $\sigma \in X_{n}$, the identity $\left\{\partial_{i} g \cdot \sigma \mid i=0, \ldots, n\right\}=\left\{g \cdot \partial_{i} \sigma \mid i=\right.$ $0, \ldots, n\}$ holds. In this way are defined permutation representations $\rho_{\sigma}$ : $G \rightarrow \Sigma_{n+1}$, which we require to be constant on $G$-orbits, i.e. $\rho_{\sigma}(g)=$ $\rho_{g^{\prime} \cdot \sigma}(g)$, for all $g, g^{\prime} \in G$.

ii) By the above condition, an action of $G$ on the set $\coprod_{n \geq 0} X_{n} \times \Delta_{n}$ is defined, for $(\sigma, x) \in X_{n} \times \Delta_{n}$ and $g \in G$, letting: $g \cdot(\sigma, x):=\left(g \cdot \sigma, \rho_{\sigma}(g)(x)\right)$. We then require that this action be compatible with the equivalence relation " " which defines the geometric realization $\left|X_{\bullet}\right|:=\coprod_{n \geq 0} X_{n} \times \Delta_{n} / \sim$.

If, for $n \geq 0$, the actions $G \times X_{n} \rightarrow X_{n}$ commute with the face and degeneracy operators, then $X_{\bullet}$ is said to be a simplicial $G$-set. In this case the quotient sets $X_{n} / G$, for $n \geq 0$, together with the induced face and degeneracy operators, form a simplicial set $X_{\bullet} / G$. Moreover, since the representations $\rho_{\sigma}$ are trivial for all $\sigma \in X_{\bullet}$, there is a natural isomorphism $\left|X_{\bullet}\right| / G \cong\left|X_{\bullet} / G\right|$.

Let $G$ be a profinite group and $X \bullet$ a simplicial profinite set (i.e. a simplicial object in the category of profinite sets and their continuous maps). We define a continuous geometric action of $G$ on $X$ • to be a geometric action such that each action $G \times X_{n} \rightarrow X_{n}$ is continuous and with open orbits for $n \geq 0$. Moreover, we require that there exists an open subgroup $U \leq G$ such that $X_{\bullet}$, with the induced $U$-action, is a simplicial $U$-set.

Let $\left\{G^{\lambda}\right\}_{\lambda \in \Lambda}$ be the tower of open subgroups of $G$ contained in $U$. Then, for all $\lambda \in \Lambda$, the quotient $X_{\bullet} / G^{\lambda}$ is a simplicial discrete finite set endowed with a geometric continuous action of $G$.

Now let $G \rightarrow G^{\prime}$ be a homomorphism of a discrete group in a profinite group, with dense image, and let $\left\{G^{\lambda}\right\}_{\lambda \in \Lambda}$ be the tower of subgroups of $G$ which are inverse images of open subgroups of $G^{\prime}$. Let $X$. be a simplicial set endowed with a geometric $G$-action such that the set of $G$-orbits in $X_{n}$ is finite for all $n \geq 0$. Assume, moreover, that there exists a $\mu \in \Lambda$ such that $X_{\bullet}$, with the induced $G^{\mu}$ action, is a simplicial $G^{\mu}$-set. 
The $G^{\prime}$-completion of $X \bullet$ is defined to be the simplicial profinite set,

$$
X_{\bullet}^{\prime}:=\lim _{\overleftarrow{\lambda \in \Lambda}} X_{\bullet} / G^{\lambda},
$$

which is then endowed with a natural continuous geometric action of $G^{\prime}$. The simplicial profinite set $X_{\bullet}^{\prime}$ has the following universal property. Let $f: X_{\bullet} \rightarrow Y_{\bullet}$ be a simplicial $G$-equivariant map, where $Y_{\bullet}$ is a simplicial profinite set endowed with a continuous geometric action of $G^{\prime}$. Then $f$ factors uniquely through the natural map $X_{\bullet} \rightarrow X_{\bullet}^{\prime}$ and a simplicial $G^{\prime}$-equivariant continuous map $f^{\prime}: X_{\bullet}^{\prime} \rightarrow Y_{\bullet}$.

Let us apply the definition of profinite $G^{\prime}$-completion of a simplicial set endowed with a geometric $G$-action to the Teichmüller group $\Gamma_{g, n}$ and its procongruence completion $\check{\Gamma}_{g, n}$. The tower of levels which defines the procongruence completion contains the abelian level $\Gamma(m)$, for $m \geq 3$. By Proposition 2.2, there is then an ordering of the vertex set of the simplicial complex $C\left(S_{g, n}\right)$ such that the associated simplicial set $C\left(S_{g, n}\right)$ • is a $\Gamma(m)$-simplicial set endowed with a natural geometric $\Gamma_{g, n}$-action. For this ordering, the conditions prescribed in order to define the $\check{\Gamma}_{g, n}$-completion of the simplicial set $C\left(S_{g, n}\right)$ • are all satisfied.

Definition 3.2. $\quad$ i) For $2 g-2+n>0$, the simplicial profinite set $\check{C}\left(S_{g, n}\right)$. is defined to be the $\check{\Gamma}_{g, n}$-completion of the simplicial set $C\left(S_{g, n}\right)$ •.

ii) Let us define a simplicial profinite complex to be an abstract simplicial complex whose set of vertices is endowed with a profinite topology such that the sets of $k$-simplices, with the induced topologies, are compact and then profinite, for all $k \geq 0$. For these simplicial complexes, the procedure which associates to an abstract simplicial complex and an ordering of its vertex set a simplicial set produces a simplicial profinite set. In Remarks 7.4 and 7.8 in [9], it is shown that $\check{C}\left(S_{g, n}\right)$ • is the simplicial profinite set associated to a simplicial profinite complex $\check{C}\left(S_{g, n}\right)$, which we call the procongruence curve complex.

By definition, there is a natural $\Gamma_{g, n}$-equivariant map of simplicial complexes $i_{*}: C\left(S_{g, n}\right) \rightarrow \check{C}\left(S_{g, n}\right)$. From Proposition 5.1 in [6] and the fact that the natural homomorphism $i: \Gamma_{g, n} \rightarrow \check{\Gamma}_{g, n}$ is injective, it follows:

Proposition 3.3. For $2 g-2+n>0$, the natural map $i_{*}: C\left(S_{g, n}\right) \rightarrow \check{C}\left(S_{g, n}\right)$ is injective.

By Proposition 6.5 in [6], the stabilizers for the action of an open subgroup of the procongruence Teichmüller group $\check{\Gamma}_{g, n}$ on the procongruence curve complex $\check{C}\left(S_{g, n}\right)$ are also determined:

Proposition 3.4. For $2 g-2+n>0$, let $i: \Gamma_{g, n} \hookrightarrow \check{\Gamma}_{g, n}$ be the natural monomorphism, let $\check{\Gamma}^{\lambda}$ be an open subgroup of $\check{\Gamma}_{g, n}$ and let $\Gamma^{\lambda}:=i^{-1}\left(\check{\Gamma}^{\lambda}\right)$. Then, the stabilizer $\check{\Gamma}_{\sigma}^{\lambda}$ of a simplex $\sigma \in \operatorname{Im} C\left(S_{g, n}\right) \subset C^{\prime}\left(S_{g, n}\right)$ for the action of the subgroup $\check{\Gamma}^{\lambda}$ is the closure in $\check{\Gamma}_{g, n}$ of the image of the stabilizer $\Gamma_{\sigma}^{\lambda}$ for the action of $\Gamma^{\lambda}$ on $C\left(S_{g, n}\right)$.

\section{The COMPleX OF PROFINITE CURVES}

The purpose of this section is to provide an alternative, more intrinsic, description of the simplicial profinite complex $\check{C}\left(S_{g, n}\right)$ in terms of the profinite fundamental group $\hat{\Pi}_{g, n}$ of the Riemann surface $S_{g, n}$. 
We say that a simple closed curve (s.c.c.) $\gamma$ on the Riemann surface $S_{g, n}$ is non-peripheral if it does not bound a disc with less than two punctures.

Let $\mathscr{L}_{g, n} \cong C\left(S_{g, n}\right)_{0}$, for $2 g-2+n>0$, denote the set of isotopy classes of non-peripheral simple closed curves on $S_{g, n}$. Let $\Pi_{g, n} / \sim$ be the set of conjugacy classes of elements of $\Pi_{g, n}$ and let $\mathscr{P}_{2}\left(\Pi_{g, n} / \sim\right)$ be the set of unordered pairs of elements of $\Pi_{g, n} / \sim$.

For a given $\gamma \in \Pi_{g, n}$, let us denote by $\gamma^{ \pm 1}$ the set $\left\{\gamma, \gamma^{-1}\right\}$ and by $\left[\gamma^{ \pm 1}\right]$ its equivalence class in $\mathscr{P}_{2}\left(\Pi_{g, n} / \sim\right)$. Let us then define the natural embedding $\iota: \mathscr{L}_{g, n} \hookrightarrow \mathscr{P}_{2}\left(\Pi_{g, n} / \sim\right)$, choosing, for an element $\gamma \in \mathscr{L}_{g, n}$, an element $\vec{\gamma}_{*} \in \Pi_{g, n}$ whose free homotopy class contains $\gamma$ and letting $\iota(\gamma):=\left[\vec{\gamma}_{*}^{ \pm 1}\right]$.

Let $\hat{\Pi}_{g, n} / \sim$ be the set of conjugacy classes of elements of $\hat{\Pi}_{g, n}$ and $\mathscr{P}_{2}\left(\hat{\Pi}_{g, n} / \sim\right)$ the profinite set of unordered pairs of elements of $\hat{\Pi}_{g, n} / \sim$. From combinatorial group theory (cf. [20]), it is known that the set $\Pi_{g, n} / \sim$ embeds in the profinite set $\hat{\Pi}_{g, n} / \sim$. So, let us define the set of non-peripheral profinite s.c.c.'s $\hat{\mathscr{L}}_{g, n}$ on $S_{g, n}$ to be the closure of the set $\iota\left(\mathscr{L}_{g, n}\right)$ inside the profinite set $\mathscr{P}_{2}\left(\hat{\Pi}_{g, n} / \sim\right)$. When it is clear from the context, we omit the subscripts and denote these sets simply by $\mathscr{L}$ and $\hat{\mathscr{L}}$.

There is a slight abuse of terminology here because the elements of $\mathscr{L}$ are isotopy classes of non-peripheral s.c.c.'s. Possibly, it would have been more appropriate to call the elements of $\hat{\mathscr{L}}$ isotopy classes of non-peripheral profinite s.c.c.'s. This terminology could have been, at least partially, justified in terms of the profinite completion of the Riemann surface $S_{g, n}$, but it seemed a bit too awkward and, in any case, not entirely meaningful.

The profinite set $\hat{\mathscr{L}}$ consists of equivalence classes $\left[\alpha^{ \pm 1}\right]$ of pairs of elements of $\hat{\Pi}_{g, n}$, such that $\alpha$ is the limit of a sequence in $\Pi_{g, n}$ of elements whose free homotopy classes contain s.c.c.'s on $S_{g, n}$.

An ordering of the set $\left\{\alpha, \alpha^{-1}\right\}$ is preserved by the conjugacy action and defines an orientation for the associated equivalence class $\left[\alpha^{ \pm 1}\right] \in \hat{\mathscr{L}}$.

An alternative group-theoretic description of the set of non-peripheral s.c.c.'s $\mathscr{L}$ is the following. Let $\mathscr{S}\left(\Pi_{g, n}\right) / \sim$ be the set of conjugacy classes of cyclic subgroups of $\Pi_{g, n}$. There is a natural embedding $\iota^{\prime}: \mathscr{L} \hookrightarrow \mathscr{S}\left(\Pi_{g, n}\right) / \sim$, defined by sending $\gamma \in \mathscr{L}$ to the conjugacy class of the cyclic subgroup of $\Pi_{g, n}$ generated by an element $\vec{\gamma}_{*} \in \Pi_{g, n}$ whose free homotopy class contains $\gamma$. So, let $\hat{\mathscr{S}}\left(\hat{\Pi}_{g, n}\right) / \sim$ be the profinite set of conjugacy classes of closed cyclic subgroups of $\hat{\Pi}_{g, n}$. There is a natural embedding of $\mathscr{S}\left(\Pi_{g, n}\right) / \sim$ in $\hat{\mathscr{S}}\left(\hat{\Pi}_{g, n}\right) / \sim$, and we define $\hat{\mathscr{L}}^{\prime}$ to be the closure of $\iota^{\prime}(\mathscr{L})$ inside $\hat{\mathscr{S}}\left(\hat{\Pi}_{g, n}\right) / \sim$. There is a natural continuous surjective map $\hat{\mathscr{L}} \rightarrow \hat{\mathscr{L}}^{\prime}$. However, it is not yet clear whether this map is also injective.

For all $k \geq 0$, there are natural embeddings of the sets of $k$-simplices of the curve complex $C\left(S_{g, n}\right)$ into the profinite sets $\mathscr{P}_{k+1}(\hat{\mathscr{L}})$ and $\mathscr{P}_{k+1}\left(\hat{\mathscr{L}}^{\prime}\right)$ of unordered subsets of $k+1$ elements of $\hat{\mathscr{L}}$ and $\hat{\mathscr{L}}^{\prime}$, respectively. Let us then define:

Definition 4.1. Let $L\left(\hat{\Pi}_{g, n}\right)$ and $L^{\prime}\left(\hat{\Pi}_{g, n}\right)$, for $2 g-2+n>0$, be the simplicial profinite complexes whose set of $k$-simplices is the closure of the set of $k$-simplices of the curve complex $C\left(S_{g, n}\right)$ inside, respectively, the profinite sets $\mathscr{P}_{k+1}(\hat{\mathscr{L}})$ and $\mathscr{P}_{k+1}\left(\hat{\mathscr{L}}^{\prime}\right)$, for $k \geq 0$. The simplicial profinite complex $L\left(\hat{\Pi}_{g, n}\right)$ is called the complex of profinite curves on $S_{g, n}$. 
By their definition, the simplicial profinite complexes defined above admit a natural continuous action of the procongruence Teichmüller group $\check{\Gamma}_{g, n}$ and are linked by a natural continuous $\check{\Gamma}_{g, n}$-equivariant surjective map $L\left(\hat{\Pi}_{g, n}\right) \rightarrow L^{\prime}\left(\hat{\Pi}_{g, n}\right)$.

The embeddings $C\left(S_{g, n}\right) \hookrightarrow L\left(\hat{\Pi}_{g, n}\right)$ and $C\left(S_{g, n}\right) \hookrightarrow L^{\prime}\left(\hat{\Pi}_{g, n}\right)$ have dense images and are $\Gamma_{g, n}$-equivariant. In particular, there are only finitely many $\check{\Gamma}_{g, n}$-orbits of $k$-simplices in $L\left(\hat{\Pi}_{g, n}\right)$ and $L^{\prime}\left(\hat{\Pi}_{g, n}\right)$, for all $k \geq 0$, and each of them contains a discrete representative.

By the universal property of the $\check{\Gamma}_{g, n}$-completion, for every $k \geq 0$ there is a series of natural surjective continuous $\check{\Gamma}_{g, n}$-equivariant maps of profinite sets:

$$
\check{C}\left(S_{g, n}\right)_{k} \rightarrow L\left(\hat{\Pi}_{g, n}\right)_{k} \rightarrow L^{\prime}\left(\hat{\Pi}_{g, n}\right)_{k} .
$$

These maps are clearly compatible with the respective structures of simplicial complexes and induce a series of natural surjective continuous maps of simplicial profinite complexes.

Theorem 4.2. For $2 g-2+n>0$, there is a series of natural $\check{\Gamma}_{g, n}$-equivariant isomorphisms of simplicial profinite complexes: $\check{C}\left(S_{g, n}\right) \stackrel{\sim}{\rightarrow} L\left(\hat{\Pi}_{g, n}\right) \stackrel{\sim}{\rightarrow} L^{\prime}\left(\hat{\Pi}_{g, n}\right)$.

Proof. It is clearly enough to prove that the composition of the two maps above, which we call $\Phi_{\bullet}$, is an isomorphism. Since, in a simplicial complex, simplices are determined by their faces, it is enough to prove that $\Phi_{0}$ is injective.

In order to prove the claim, we will show that the actions of $\check{\Gamma}_{g, n}$ on $\check{C}\left(S_{g, n}\right)_{0}$ and on $L^{\prime}\left(\hat{\Pi}_{g, n}\right)_{0}$ have the same orbits and the same stabilizers. Before we proceed further, let us give a geometric description of the natural faithful representations, introduced in Section 3 .

$$
\check{\rho}_{g, n}: \check{\Gamma}_{g, n} \hookrightarrow \operatorname{Out}\left(\hat{\Pi}_{g, n}\right) \quad \text { and } \quad \tilde{\rho}_{g, n}: \check{\Gamma}_{g, n+1} \hookrightarrow \operatorname{Aut}\left(\hat{\Pi}_{g, n}\right) .
$$

We are going to interpret these representations as monodromy representations associated to some profinite coverings of the universal curve over the moduli stack of stable curves (for the general theory of these spaces, see [21]).

Let $\widetilde{\mathcal{M}}$ (respectively, $\widetilde{\mathscr{C}}$ ) be the inverse limit of all representable geometric level structures over $\overline{\mathcal{M}}_{g, n}$ (respectively, over $\overline{\mathcal{M}}_{g, n+1}$ ) and let $\overline{\mathscr{C}}:=\overline{\mathcal{M}}_{g, n+1} \times \overline{\mathcal{M}}_{g, n} \widetilde{\mathcal{M}}$ be the pull-back of the universal proper curve. They are connected by the commutative diagram

$$
\begin{array}{lll}
\tilde{\mathscr{C}} & & \\
\downarrow & \searrow & \\
\frac{\mathscr{C}}{\downarrow} \rightarrow & \widetilde{\mathcal{M}} \\
\overline{\mathcal{M}}_{g, n+1} & \rightarrow & \overline{\mathcal{M}}_{g, n} .
\end{array}
$$

Let us observe that, by Definitions 2.3 and 3.2 the nerve of the cover of the D-M boundary $\partial \widetilde{\mathcal{M}}$ of the profinite covering $\widetilde{\mathcal{M}}$ by its irreducible components is described by the procongruence curve complex $\check{C}\left(S_{g, n}\right)$.

The procongruence Teichmüller group $\check{\Gamma}_{g, n}$ identifies with the deck transformation groups of the profinite Galois coverings $\widetilde{\mathcal{M}} \rightarrow \overline{\mathcal{M}}_{g, n}$ and $\overline{\mathscr{C}} \rightarrow \overline{\mathcal{M}}_{g, n+1}$, while the procongruence Teichmüller group $\check{\Gamma}_{g, n+1}$ identifies with the deck transformation group of the profinite Galois covering $\widetilde{\mathscr{C}} \rightarrow \overline{\mathcal{M}}_{g, n+1}$. 
Moreover, the kernel $\hat{\Pi}_{g, n}$ of the natural epimorphism $\check{\Gamma}_{g, n+1} \rightarrow \check{\Gamma}_{g, n}$ consists of the deck transformations of the covering $\widetilde{\mathscr{C}} \rightarrow \overline{\mathcal{M}}_{g, n+1}$ which preserve the fibers of the natural morphism $\widetilde{\mathscr{C}} \rightarrow \widetilde{\mathcal{M}}$. In this way, the profinite group $\hat{\Pi}_{g, n}$ is canonically identified, modulo inner automorphisms, with the logarithmic algebraic fundamental groups of the fibers of the morphism $\overline{\mathscr{C}} \rightarrow \widetilde{\mathcal{M}}$, with respect to the logarithmic structure associated to the divisor of marked and singular points.

A good reference for the general theory of algebraic fundamental groups of log schemes is Hoshi's paper 14. However, these groups, at least for a stable marked complex projective curve $C$, can be described in a more elementary fashion.

Let $\hat{C}$ be the real oriented blow-up of $C$ along the divisor of marked and singular points. Then, $\hat{C}$ is a compact orientable Riemann surface with boundary and the blow-up map $\hat{C} \rightarrow C$ contracts a set of disjoint non-peripheral s.c.c.'s on $\hat{C}$ onto the nodes of $C$ and its boundary components onto the marked points of $C$.

Let $C^{\log }$ be the curve $C$ endowed with the logarithmic structure associated to the divisor of marked and singular points. A morphism $X^{\log } \rightarrow C^{\log }$ of logarithmic schemes is $\log$ étale if and only if the underlying morphism of schemes $X \rightarrow C$ pulls back to a topological covering map $X \times{ }_{C} \hat{C} \rightarrow \hat{C}$ via the blow-up map $\hat{C} \rightarrow C$.

The algebraic fundamental group $\hat{\pi}_{1}\left(C^{\log }\right)$ then classifies finite coverings of $C^{\log }$ with the above property, and therefore it is naturally isomorphic to the profinite completion of the topological fundamental group of the Riemann surface $\hat{C}$.

The representation $\tilde{\rho}_{g, n}: \check{\Gamma}_{g, n+1} \rightarrow \operatorname{Aut}\left(\hat{\Pi}_{g, n}\right)$ can be described as follows. For $x \in \widetilde{\mathcal{M}}$, let $\widetilde{\mathscr{C}}_{x}$ and $\overline{\mathscr{C}}_{x}$ be, respectively, the fibers of the morphisms $\widetilde{\mathscr{C}} \rightarrow \widetilde{\mathcal{M}}$ and $\overline{\mathscr{C}} \rightarrow \widetilde{\mathcal{M}}$. For all $x \in \widetilde{\mathcal{M}}$, the profinite group $\hat{\Pi}_{g, n}$ identifies with the deck transformation group of the profinite covering $\widetilde{\mathscr{C}}_{x} \rightarrow \overline{\mathscr{C}}_{x}$. Let us denote by $\bar{f}$ the image of $f \in \check{\Gamma}_{g, n+1}$ via the natural epimorphism $\check{\Gamma}_{g, n+1} \rightarrow \check{\Gamma}_{g, n}$. The element $f \in$ $\check{\Gamma}_{g, n+1}$ induces, by restriction, an isomorphism of profinite curves $f: \widetilde{\mathscr{C}}_{x} \rightarrow \widetilde{\mathscr{C}}_{\bar{f}(x)}$. The automorphism $\tilde{\rho}_{g, n}(f): \hat{\Pi}_{g, n} \rightarrow \hat{\Pi}_{g, n}$ is then defined, for $\alpha \in \hat{\Pi}_{g, n}$, by the assignment $\tilde{\rho}_{g, n}(f)(\alpha)=f^{-1} \alpha f$.

The profinite universal monodromy representation $\check{\rho}_{g, n}: \check{\Gamma}_{g, n} \rightarrow \operatorname{Out}\left(\hat{\Pi}_{g, n}\right)$ is the outer representation associated to $\tilde{\rho}_{g, n}$. However, in the present geometric context, there is a more direct way to describe $\check{\rho}_{g, n}$.

The profinite group $\check{\Gamma}_{g, n}$ identifies with the deck transformation group of the profinite covering $\overline{\mathscr{C}} \rightarrow \overline{\mathcal{M}}_{g, n+1}$. Let us denote by $\overline{\mathscr{C}}_{x}^{\log }$ and $\overline{\mathscr{C}}_{f(x)}^{\log }$ the fibers of the curve $\overline{\mathscr{C}} \rightarrow \widetilde{\mathcal{M}}$ endowed with the logarithmic structure associated to the divisor of marked and singular points. An element $f \in \check{\Gamma}_{g, n}$ determines by restriction an isomorphism of logarithmic algebraic curves $f: \overline{\mathscr{C}}_{x}^{\log } \rightarrow \overline{\mathscr{C}}_{f(x)}^{\log }$ and then of logarithmic algebraic fundamental groups $f_{\natural}: \hat{\pi}_{1}\left(\overline{\mathscr{C}}_{x}^{\log }\right) \rightarrow \hat{\pi}_{1}\left(\overline{\mathscr{C}}_{f(x)}^{\log }\right)$. These groups identify, modulo inner automorphisms, with the profinite group $\hat{\Pi}_{g, n}$. Therefore, the isomorphism $f_{\natural}$ determines an element of $\operatorname{Out}\left(\hat{\Pi}_{g, n}\right)$.

The discrete universal monodromy representation $\rho: \Gamma_{g, n} \rightarrow \operatorname{Out}\left(\Pi_{g, n}\right)$ can be described in a similar way, where the Bers bordifications $\bar{T}_{g, n}$ and $\bar{T}_{g, n+1}$ and the universal Teichmüller curve $\overline{\mathfrak{C}} \rightarrow \bar{T}_{g, n}$ play the same role of the inverse limit spaces $\widetilde{\mathcal{M}}, \tilde{\mathscr{C}}$ and $\overline{\mathscr{C}}$.

There are natural injective maps $\bar{T}_{g, n} \hookrightarrow \widetilde{\mathcal{M}}, \bar{T}_{g, n+1} \hookrightarrow \widetilde{\mathscr{C}}$ and $\overline{\mathfrak{C}} \hookrightarrow \overline{\mathscr{C}}$. For $x \in \widetilde{\mathcal{M}}$ in the image of $\bar{T}_{g, n}$, the fiber $\overline{\mathscr{C}}_{x}$ is contained in the image of $\overline{\mathfrak{C}}$ and the 
logarithmic topological fundamental group of $\overline{\mathscr{C}}_{x}$ (here, by this, we just mean the topological fundamental group of the real oriented blow-up of $\overline{\mathscr{C}}_{x}$ along its marked and singular points) identifies, modulo inner automorphisms, with $\Pi_{g, n}$.

For a given s.c.c. $\gamma \in \mathscr{L}^{\prime}$, let $\left(C_{\gamma}, \psi\right) \in \partial T_{g, n}$ be such that $C_{\gamma}$ is a stable $n$ pointed, genus $g$ curve with a single node and the degenerate marking $\psi: S_{g, n} \rightarrow C_{\gamma}$ sends $\gamma$ to the node. Then let $x \in \widetilde{\mathcal{M}}$ be the image of the point $\left(C_{\gamma}, \psi\right)$ via the natural embedding $\bar{T}_{g, n} \hookrightarrow \widetilde{\mathcal{M}}$ and identify the stable curve $C_{\gamma}$ with the fiber $\overline{\mathscr{C}}_{x}$.

Let $\tilde{C}_{\gamma}$ be the inverse limit of all logarithmic étale coverings $\left\{C_{\gamma}^{\lambda}\right\}_{\lambda \in \Lambda}$ of $C_{\gamma}$, with respect to the logarithmic structure associated to the divisor of marked and singular points. Let $\left\{R^{\lambda} \subset C_{\gamma}^{\lambda}\right\}_{\lambda \in \Lambda}$ be an inverse system of irreducible components. Then, we call the inverse limit $\lim _{\lambda} R^{\lambda}$ an irreducible component of $\tilde{C}_{\gamma}$. Similarly, for $\left\{n^{\lambda} \subset C_{\gamma}^{\lambda}\right\}_{\lambda \in \Lambda}$ an inverse system of nodes, we call $\lim _{\lambda} n^{\lambda}$ a node of $\tilde{C}_{\gamma}$. For all $\lambda$, the curve $C_{\gamma}^{\lambda}$ is semi-stable. In particular, each of its nodes is contained in exactly two irreducible components. Then, the same property holds for the inverse $\operatorname{limit} \tilde{C}_{\gamma}$.

The curve $\tilde{C}_{\gamma}$ identifies with the fiber $\tilde{\mathscr{C}}_{x}$ of the curve $\tilde{\mathscr{C}} \rightarrow \widetilde{\mathcal{M}}$. In this way, the group of deck transformations of the covering $\tilde{C}_{\gamma} \rightarrow C_{\gamma}$ identifies with $\hat{\Pi}_{g, n}$.

From the above description, it is clear that two discrete s.c.c. $\gamma, \gamma^{\prime} \in \mathscr{L}^{\prime} \subset \hat{\mathscr{L}}^{\prime}$ are in the same $\check{\Gamma}_{g, n}$-orbit if and only if the associated stable curves $C_{\gamma}$ and $C_{\gamma^{\prime}}$ have the same topological type. This already implies that the natural continuous $\check{\Gamma}_{g, n}$-equivariant map $\Phi_{0}: \check{C}\left(S_{g, n}\right)_{0} \rightarrow L^{\prime}\left(\hat{\Pi}_{g, n}\right)_{0}$ induces a bijection between the $\check{\Gamma}_{g, n}$-orbits of the two sets.

It remains to prove that $\Phi_{0}$ induces an isomorphism between the respective stabilizers. A $\check{\Gamma}_{g, n}$-orbit in $\check{C}\left(S_{g, n}\right)_{0}$ contains simplices in the image of the discrete complex $C\left(S_{g, n}\right)_{0}$. Hence, it is enough to show that, for a given s.c.c. $\gamma$ on the Riemann surface $S_{g, n}$, the stabilizer $\check{\Gamma}_{\gamma}$ of its image in $\check{C}\left(S_{g, n}\right)_{0}$ equals the stabilizer $\check{\Gamma}_{\Phi_{0}(\gamma)}$ of its image in $\hat{\mathscr{L}}^{\prime}$.

We are going to make use of the description of the simplicial profinite set $\check{C}\left(S_{g, n}\right)$. in terms of framed, marked, graph decompositions of the homology of coverings of $S_{g, n}$ given in Section 7 of [9]. Let us briefly recall the construction given there, while, for more details and precise definitions, we refer to Sections 6 and 7 of $[9$.

Let $K$ be a characteristic finite index subgroup of $\Pi_{g, n}$ with associated covering $p_{K}: S_{K} \rightarrow S_{g, n}$ and covering transformation group $G_{K}$. Let $n_{K}$ be the number of punctures on the Riemann surface $S_{K}$.

In Section 6 of [9], the author described the nerve of the D-M boundary of the abelian level structure $\mathcal{M}\left(S_{K}\right)^{(m)}$ over the moduli stack $\mathcal{M}\left(S_{K}\right)$, which parametrizes complex curves homeomorphic to $S_{K}$.

A $k$-simplex $\sigma=\left\{\gamma_{e}\right\}_{e \in E}$ of the curve complex $C\left(S_{K}\right)$ determines a stratum $\delta_{\sigma}^{(m)}$ of codimension $k+1$ in the D-M boundary of $\overline{\mathcal{M}}\left(S_{K}\right)^{(m)}$. The s.c.c.'s in $\sigma$ determine a partition $\amalg_{v \in V} S_{v}:=S_{K} \backslash \sigma$ of the Riemann surface $S_{K}$ in open connected subsurfaces $S_{v}$ of negative Euler characteristic.

In its turn, this partition determines what we call a framed, $n_{K}$-marked, graph decomposition of the homology group $H_{1}\left(\bar{S}_{K}, \mathbb{Z} / m\right.$ ) (cf. Definitions 6.1 and 6.5 in [9]). This is the graph $\left(D, Y_{\sigma}\right)$ of abelian subgroups of $H_{1}\left(\bar{S}_{K}, \mathbb{Z} / m\right)$ whose vertex groups $D_{v}$, for $v \in V$, consist of the images of the natural homomorphisms $H_{1}\left(S_{v}, \mathbb{Z} / 2\right) \rightarrow H_{1}\left(\bar{S}_{K}, \mathbb{Z} / m\right)$ and whose edge groups $D_{e}$, for $e \in E$, are the cyclic 
subgroups of $H_{1}\left(\bar{S}_{K}, \mathbb{Z} / m\right)$ determined by the s.c.c.'s $\gamma_{e} \in \sigma$. Let us observe that the graph $Y_{\sigma}$ is just the dual graph of the stable degeneration of the Riemann surface $S_{K}$ whose vanishing cycles are the s.c.c.'s in $\sigma$.

Let $\mathscr{P}_{n_{K}}$ be the set of subsets of the index set $\left\{1, \ldots, n_{K}\right\}$. Let us then define the marking $d: V \rightarrow \mathscr{P}_{n_{K}}$ assigning to a vertex $v$ of the graph $Y_{\sigma}$ the indices of the punctures lying on the corresponding subsurface $S_{v}$ of $S_{K}$. The frame epimorphism $\mu_{e}: \mathbb{Z} \rightarrow D_{e}$, for an edge $e \in E$ of the graph $Y_{\sigma}$, is the natural epimorphism between the two cyclic subgroups associated to the s.c.c. $\gamma_{e}$ in the homology groups $H_{1}\left(\bar{S}_{K}, \mathbb{Z}\right)$ and $H_{1}\left(\bar{S}_{K}, \mathbb{Z} / m\right)$, respectively.

By Theorem 6.7 of $\left[9\right.$, for $m \geq 2$, the framed, $n_{K}$-marked, graph decomposition $\left(D, Y_{\sigma}, d,\left\{\mu_{e}\right\}\right)$, modulo a natural equivalence relation, determines the stratum $\delta_{\sigma}^{(m)}$ associated to the simplex $\sigma$.

A simplex $\sigma \in C\left(S_{g, n}\right)$, via the covering $p_{K}: S_{K} \rightarrow S_{g, n}$, determines the simplex $\sigma^{\prime}:=p_{K}^{-1}(\sigma)$ in the curve complex $C\left(S_{K}\right)$. By the above construction, we then associate to $\sigma$ the equivalence class $\left[D, Y_{\sigma^{\prime}}, d,\left\{\mu_{e}\right\}\right]$ of the framed, $n_{K}$-marked, graph decomposition of $H_{1}\left(\bar{S}_{K}, \mathbb{Z} / m\right)$ determined by the simplex $\sigma^{\prime}$. This decomposition has the further property of admitting a natural action of the covering transformation group $G_{K}$.

The author defined the simplicial finite set $\mathscr{G}_{G_{K}}^{n_{K}}\left(H_{1}\left(\bar{S}_{K}, \mathbb{Z} / m\right)\right)$, for $m \geq 2$, of framed, $n_{K}$-marked, graph decompositions of the homology group $H_{1}\left(\bar{S}_{K}, \mathbb{Z} / m\right)$, endowed with a natural $G_{K}$-action in Section 7 of [9]. As observed in iv), Remark 7.5 of [9], if the subgroup $K$ satisfies the hypotheses of Theorem 3.9 of [9], the natural map defined above,

$$
\mathfrak{g}_{K,(m)}: C\left(S_{g, n}\right) \bullet \rightarrow \mathscr{G}_{G_{K}}^{n_{K}}\left(H_{1}\left(\bar{S}_{K}, \mathbb{Z} / m\right)\right) \bullet
$$

is $\Gamma_{g, n}$-equivariant and factors through a continuous $\check{\Gamma}_{g, n}$-equivariant map:

$$
\check{\mathfrak{g}}_{K,(m)}: \check{C}\left(S_{g, n}\right) \bullet \rightarrow \mathscr{G}_{G_{K}}^{n_{K}}\left(H_{1}\left(\bar{S}_{K}, \mathbb{Z} / m\right)\right)_{\bullet} .
$$

By Corollary 7.7 of $[9$, if $\{K\}$ is a cofinal system of finite index subgroups of $\Pi_{g, n}$ satisfying the hypotheses of Theorem 3.9 of [9] and $m$ is an integer $\geq 2$, there is a natural continuous, injective, $\check{\Gamma}_{g, n}$-equivariant map of simplicial profinite sets:

$$
\prod_{K} \check{\mathfrak{g}}_{K,(m)}: \check{C}\left(S_{g, n}\right) \bullet \hookrightarrow \prod_{K} \mathscr{G}_{G_{K}}^{n_{K}}\left(H_{1}\left(\bar{S}_{K}, \mathbb{Z} / m\right)\right) \bullet
$$

The existence of such a cofinal system is then ensured by Lemma 3.10 of [9].

As observed in Remark 6.8 of $[9]$, with $\mathbb{Z} / 2$-coefficients, all framings of the edge groups are equivalent, i.e. a simplex of $\mathscr{G}_{G_{K}}^{n_{K}}\left(H_{1}\left(\bar{S}_{K}, \mathbb{Z} / 2\right)\right)$ • is already determined by the equivalence class of the corresponding $n_{K}$-marked, graph decomposition.

So, let us describe the map $\check{\mathfrak{g}}_{K,(2)}$ for a given s.c.c. $\gamma$ on the Riemann surface $S_{g, n}$. The s.c.c. $\gamma$ determines a partition $\amalg_{v \in V} S_{v}:=S_{K} \backslash p_{K}^{-1}(\gamma)$ of the Riemann surface $S_{K}$, and this determines, as above, an $n_{K}$-marked, graph $\left(D^{K}, Y^{K}, d^{K}\right)$ of abelian subgroups of $H_{1}\left(\bar{S}_{K}, \mathbb{Z} / 2\right)$. This comes with a natural $G_{K}$-action, induced by the deck transformations of the covering map $p_{K}: S_{K} \rightarrow S_{g, n}$.

The map $\check{\mathfrak{g}}_{K,(2)}$ then associates to the 0-simplex of $\check{C}\left(S_{g, n}\right)$ • associated to the s.c.c. $\gamma$ on $S_{g, n}$ the corresponding equivalence class $\left[D^{K}, Y^{K}, d^{K}\right]$ of $n_{K}$-marked, graph decomposition of the homology group $H_{1}\left(\bar{S}_{K}, \mathbb{Z} / 2\right)$, endowed with a natural $G_{K}$-action. 
By Lemma 3.10 of [9], we can assume that all elements in the inverse system $\{K\}$ satisfy the hypotheses of Theorem 3.9 of [9], i.e. they are such that the inverse image $p_{K}^{-1}(\gamma)$ contains no separating curves or cut pairs, so that the cyclic subgroups of the homology group $H_{1}\left(\bar{S}_{K}, \mathbb{Z} / 2\right)$ determined by the s.c.c.'s contained in $p_{K}^{-1}(\gamma)$ are all distinct and non-trivial.

In order to prove that $\Phi_{0}$ induces an isomorphism between the respective stabilizers of 0 -simplices, we then have to show that the stabilizer $\check{\Gamma}_{\Phi_{0}}(\gamma)$ preserves the equivalence class $\left[D^{K}, Y^{K}, d^{K}\right]$, associated to the s.c.c. $\gamma$, for a cofinal system of characteristic finite index subgroups $\{K\}$ of $\Pi_{g, n}$ satisfying the hypotheses of Theorem 3.9 of $[9$.

Let us again consider the covering $\tilde{C}_{\gamma} \rightarrow C_{\gamma}$ whose deck transformation group was identified with $\hat{\Pi}_{g, n}$ via the degenerate marking $\psi: S_{g, n} \rightarrow C_{\gamma}$.

Let $\gamma_{*}$ be an element of the fundamental group $\Pi_{g, n}$ which generates a cyclic subgroup in the equivalence class of the given $\gamma \in \mathscr{L}^{\prime}$. Elements of $\hat{\Pi}_{g, n}$ in the conjugacy class of $\gamma_{*}$ are in natural bijective correspondence with the set $\mathcal{N}_{\gamma}$ of nodes of $\tilde{C}_{\gamma}$. Then let $\left\{\gamma_{s}\right\}_{s \in \mathcal{N}_{\gamma}}$ be the set of conjugates of $\gamma_{*}$ inside $\hat{\Pi}_{g, n}$.

The stabilizer of the node $s \in \mathcal{N}_{\gamma}$ for the action by $\hat{\Pi}_{g, n}$ is the closed cyclic group $I_{s}$ generated by $\gamma_{s}$. Moreover, the action of $I_{s}$ on the curve $\tilde{C}_{\gamma}$ has only the node $s$ as a fixed point. Therefore, the subgroups $\left\{I_{s}\right\}_{s \in c_{\gamma}}$ are their own normalizers in $\hat{\Pi}_{g, n}$. The same argument clearly applies to the stabilizers of marked points of $\tilde{C}_{\gamma}$. In particular, it holds:

Lemma 4.3. Let $1 \neq x \in \Pi_{g, n}$, for $2 g-2+n>0$, be representable by an s.c.c. on $S_{g, n}$. Then, the closed subgroup generated by $x^{m}$ in $\hat{\Pi}_{g, n}$, for $m \in \hat{\mathbb{Z}} \backslash\{0\}$, has for a normalizer the closed subgroup generated by $x$. Moreover, for $y \in \hat{\Pi}_{g, n} \backslash \overline{\langle x\rangle}$, it holds that $\overline{\langle x\rangle} \cap y \overline{\langle x\rangle} y^{-1}=\{1\}$.

Let us now assume that the s.c.c. $\gamma$ is non-separating; the other case can be treated similarly. Let $\dot{C}_{\gamma}$ be the smooth curve obtained by removing the singular point from $C_{\gamma}$. Then, $\dot{C}_{\gamma}$ has just one connected component. For suitable choices of base points, the fundamental group of the subsurface $\psi^{-1}\left(\dot{C}_{\gamma}\right)$ of $S_{g, n}$ can be identified with a subgroup $\Pi_{\gamma}$ of the fundamental group $\Pi_{g, n}$ which contains $\gamma_{*}$.

The profinite completion $\hat{\Pi}_{\gamma}$ of $\Pi_{\gamma}$ is identified with the deck transformation group of the restriction of the covering $\tilde{C}_{\gamma} \rightarrow C_{\gamma}$ to one of the two irreducible components of $\tilde{C}_{\gamma}$ which contain the node $n_{*}$ corresponding to $\gamma_{*}$. There is then a bijective correspondence between irreducible components of $\tilde{C}_{\gamma}$ and conjugates of the subgroup $\hat{\Pi}_{\gamma}$ in $\hat{\Pi}_{g, n}$. In particular, the subgroup $\hat{\Pi}_{\gamma}$ is its own normalizer in $\hat{\Pi}_{g, n}$. Let us denote by $\hat{\Pi}_{\gamma}^{\prime}$ the conjugate of $\hat{\Pi}_{\gamma}$ corresponding to the other irreducible component of $\tilde{C}_{\gamma}$ which also contains the node $n_{*}$.

Let $f \in \check{\Gamma}_{g, n}$ be an element which preserves the conjugacy class of the closed subgroup $\overline{\left\langle\gamma_{*}\right\rangle}$ of $\hat{\Pi}_{g, n}$ generated by $\gamma_{*}$ and let $\tilde{f} \in \operatorname{Aut}\left(\hat{\Pi}_{g, n}\right)$ be a lift of $f$ such that it holds $\tilde{f}\left(\overline{\left\langle\gamma_{*}\right\rangle}\right)=\overline{\left\langle\gamma_{*}\right\rangle}$. The isomorphism between fibers $\tilde{f}: \widetilde{\mathscr{C}}_{x} \rightarrow \widetilde{\mathscr{C}}_{\tilde{f}(x)}$, induced by $\tilde{f}$, then sends the irreducible component of $\widetilde{\mathscr{C}}_{x}$ stabilized by the subgroup $\hat{\Pi}_{\gamma}$ of $\hat{\Pi}_{g, n}$ to the irreducible component of $\widetilde{\mathscr{C}}_{\tilde{f}(x)}$ stabilized by either $\hat{\Pi}_{\gamma}$ or $\hat{\Pi}_{\gamma}^{\prime}$. This means that $\tilde{f}$ either preserves both subgroups $\hat{\Pi}_{\gamma}$ and $\hat{\Pi}_{\gamma}^{\prime}$ or swaps them. 
Let $\hat{K}$ be the profinite completion of a subgroup $K$ of $\Pi_{g, n}$ satisfying the hypotheses of Theorem 3.9 of [9]. The conjugacy class of the closed subgroup $\hat{K} \cap \overline{\left\langle\gamma_{*}\right\rangle}$ of $\hat{\Pi}_{g, n}$ is mapped by the series of natural epimorphisms $H_{1}(\hat{K}) \cong H_{1}\left(S_{K}\right) \rightarrow H_{1}\left(\bar{S}_{K}, \mathbb{Z} / 2\right)$ onto the $G_{K}$-orbit of the edge groups of $\left(D^{K}, Y^{K}, d^{K}\right)$, while the conjugacy class of the closed subgroups $\hat{K} \cap \hat{\Pi}_{\gamma}$ and $\hat{K} \cap \hat{\Pi}_{\gamma}^{\prime}$ is mapped onto the $G_{K}$-orbit of the vertex groups of $\left(D^{K}, Y^{K}, d^{K}\right)$.

It follows that the given $f \in \check{\Gamma}_{\Phi_{0}(\gamma)}$ preserves vertex and edge groups of the graph of groups $\left(D^{K}, Y^{K}\right)$. Under the current hypotheses, the equivalence class $\left[D^{K}, Y^{K}\right]$ of $\left(D^{K}, Y^{K}\right)$ is determined by its vertex and edge groups. Therefore, the action of $f$ preserves at least the set of simplices of $\mathscr{G}_{G_{K}}^{n_{K}}\left(H_{1}\left(\bar{S}_{K}, \mathbb{Z} / 2\right)\right) \bullet$ whose underlying graph decompositions are equivalent to the graph of groups $\left(D^{K}, Y^{K}\right)$.

On the other hand, the $G_{K^{-}}$orbit of markings for the vertices of $Y^{K}$ is obviously preserved. Thus, in conclusion, the equivalence class $\left[D^{K}, Y^{K}, d^{K}\right]$ of $\left(D^{K}, Y^{K}, d^{K}\right)$ is preserved by the action of $f$. From Corollary 7.7 in [9], it then follows that $f$ preserves the 0 -simplex of $\check{C}\left(S_{g, n}\right)_{0}$ determined by $\gamma$. This concludes the proof of Theorem 4.2

Thanks to Theorem 4.2, the procongruence curve complex $\check{C}\left(S_{g, n}\right)$ is realized in terms of profinite s.c.c.'s on $S_{g, n}$. From now on, we will identify the procongruence curve complex $\check{C}\left(S_{g, n}\right)$ with the complex of profinite curves $L\left(\hat{\Pi}_{g, n}\right)$.

The orbits of the complex of profinite curves $L\left(\hat{\Pi}_{g, n}\right)$ for the action of the procongruence Teichmüller group $\check{\Gamma}_{g, n}$ are the closures of the images of the orbits of the discrete curve complex $C\left(S_{g, n}\right)$ for the action of the Teichmüller group $\Gamma_{g, n}$ and, by the proof of Theorem 4.2 they are all distinct. The following notion is then well defined:

Definition 4.4. For $2 g-2+n>0$, let $\sigma \in L\left(\hat{\Pi}_{g, n}\right)$. The topological type of $\sigma$ is the homeomorphism type of the Riemann surface $S_{g, n} \backslash \sigma^{\prime}$, where $\sigma^{\prime} \in C\left(S_{g, n}\right)$ is such that its image in $L\left(\hat{\Pi}_{g, n}\right)$ is in the $\check{\Gamma}_{g, n}$-orbit of $\sigma$.

Let us now describe the stabilizers for the action of $\check{\Gamma}_{g, n}$ on the complex of profinite curves $L\left(\hat{\Pi}_{g, n}\right)$. Again, it is enough to describe the stabilizers of simplices in the image of the discrete curve complex. Given a simplex $\sigma \in C\left(S_{g, n}\right)$, let us denote also by $\sigma$ its image in $L\left(\hat{\Pi}_{g, n}\right)$ and by $\check{\Gamma}_{\sigma}$ the corresponding $\check{\Gamma}_{g, n}$-stabilizer.

Theorem 4.5. Let $\sigma \in L\left(\hat{\Pi}_{g, n}\right)$, for $2 g-2+n>0$, be the image of a simplex of $C\left(S_{g, n}\right)$ determined by the set $\left\{\gamma_{0}, \ldots, \gamma_{k}\right\}$ of s.c.c. on the Riemann surface $S_{g, n}$. Suppose that

$$
S_{g, n} \backslash\left\{\gamma_{0}, \ldots, \gamma_{k}\right\} \cong S_{g_{1}, n_{1}} \amalg \ldots \amalg S_{g_{h}, n_{h}}
$$

and let $\Sigma_{\sigma^{ \pm}}$be the group of permutations on the set of oriented s.c.c.'s $\left\{\vec{\gamma}_{0}^{ \pm}, \ldots, \vec{\gamma}_{k}^{ \pm}\right\}$. Then, the stabilizer $\check{\Gamma}_{\sigma}$ of $\sigma$ in $L\left(\hat{\Pi}_{g, n}\right)$ for the action of $\check{\Gamma}_{g, n}$ fits into the two exact sequences:

$$
\begin{aligned}
1 & \rightarrow \check{\Gamma}_{\vec{\sigma}} \rightarrow \check{\Gamma}_{\sigma} \rightarrow \Sigma_{\sigma^{ \pm}}, \\
1 \rightarrow \bigoplus_{i=0}^{k} \hat{\mathbb{Z}} \cdot \tau_{\gamma_{i}} & \rightarrow \check{\Gamma}_{\vec{\sigma}} \rightarrow \check{\Gamma}_{g_{1}, n_{1}} \times \cdots \times \check{\Gamma}_{g_{h}, n_{h}} \rightarrow 1 .
\end{aligned}
$$

By Proposition 3.4 and Theorem 4.2, for a simplex $\sigma \in C\left(S_{g, n}\right)$, the stabilizer $\check{\Gamma}_{\sigma}$ of its image in $L\left(\hat{\Pi}_{g, n}\right)$ for the action of $\check{\Gamma}_{g, n}$ is the closure $\bar{\Gamma}_{\sigma}$ in the profinite group $\check{\Gamma}_{g, n}$ of the discrete stabilizer $\Gamma_{\sigma}$. 
By ii) of Theorem [2.1, the injectivity of the second map from the left in the bottom sequence of Theorem 4.5 is equivalent to the claim that Looijenga levels induce the profinite topology on the abelian subgroup $\bigoplus_{i=0}^{k} \mathbb{Z} \cdot \tau_{\gamma_{i}}$ of $\Gamma_{g, n}$.

This claim then follows from the explicit description of the local monodromy representation for Looijenga level structures, for $K$ satisfying the hypotheses of Lemma 3.10 in 9 ] and all $\ell, m \geq 2$ (cf. Proposition 3.11 in [9]), which implies

$$
\Gamma^{K_{\ell},(m)} \cap \bigoplus_{i=0}^{k} \mathbb{Z} \cdot \tau_{\gamma_{i}} \leq \bigoplus_{i=0}^{k} m \mathbb{Z} \cdot \tau_{\gamma_{i}} .
$$

By Proposition 6.6 in [6], there is a natural representation:

$$
\check{\rho}_{\sigma}: \check{\Gamma}_{\vec{\sigma}} \rightarrow \check{\Gamma}_{g_{1}, n_{1}} \times \cdots \times \check{\Gamma}_{g_{h}, n_{h}} .
$$

Thus, right exactness of the bottom sequence of Theorem 4.5 follows if we show that it holds ker $\check{\rho}_{\sigma}=\bigoplus_{i=0}^{k} \hat{\mathbb{Z}} \cdot \tau_{\gamma_{i}}$.

Proceeding by induction on the rank of the simplex, it is not difficult to reduce to the case of the 0 -simplex determined by an s.c.c. $\gamma$ on $S_{g, n}$, which is dealt with by the following lemma:

Lemma 4.6. Let $\gamma$ be an s.c.c. on $S_{g, n}$ and let $\check{\Gamma}_{\vec{\gamma}}$ be the subgroup of $\check{\Gamma}_{g, n}$ consisting of elements which fix the s.c.c. $\gamma$ and an orientation on it. It holds:

i) If $\gamma$ is a non-separating s.c.c., the profinite monodromy representation $\check{\rho}_{g, n}$ then induces a representation $\check{\rho}_{\gamma}: \check{\Gamma}_{\vec{\gamma}} \rightarrow \operatorname{Out}\left(\hat{\Pi}_{g-1, n+2}\right)$, whose kernel is topologically generated by the Dehn twist $\tau_{\gamma}$.

ii) If $\gamma$ is a separating s.c.c. and it holds that $S_{g, n} \backslash \gamma \cong S_{g_{1}, n_{1}+1} \amalg S_{g_{2}, n_{2}+1}$, the profinite monodromy representation $\check{\rho}_{g, n}$ then induces a representation $\check{\rho}_{\gamma}: \check{\Gamma}_{\vec{\gamma}} \rightarrow \operatorname{Out}\left(\hat{\Pi}_{g_{1}, n_{1}+1}\right) \times \operatorname{Out}\left(\hat{\Pi}_{g_{2}, n_{2}+1}\right)$, whose kernel is topologically generated by the Dehn twist $\tau_{\gamma}$.

Proof. Let us assume that $\gamma$ is a non-separating s.c.c. on $S_{g, n}$. The case of a separating curve can be treated similarly. Let us give to the group $\Pi_{g, n}$ the standard presentation:

$$
\Pi_{g, n}=\left\langle\alpha_{1}, \ldots, \alpha_{g}, \beta_{1}, \ldots, \beta_{g}, u_{1}, \ldots, u_{n} \mid \prod_{i=1}^{g}\left[\alpha_{i}, \beta_{i}\right] \cdot u_{n} \cdots u_{1}\right\rangle,
$$

where $u_{i}$, for $i=1, \ldots, n$, is a simple loop around the puncture $P_{i}$.

Let us assume that $\beta_{g}$ is freely isotopic to $\gamma$ and put $u_{n+1}:=\beta_{g}^{-1}$ and $u_{n+2}:=$ $\alpha_{g} \beta_{g} \alpha_{g}^{-1}$. For a suitable choice of base point, the fundamental group of $S_{g, n} \backslash \gamma$ is then identified with the subgroup $\Pi_{g-1, n+2}$ of $\Pi_{g, n}$ of the presentation:

$$
\Pi_{g-1, n+2}=\left\langle\alpha_{1}, \ldots, \alpha_{g-1}, \beta_{1}, \ldots, \beta_{g-1}, u_{1}, \ldots, u_{n+2} \mid \prod_{i=1}^{g-1}\left[\alpha_{i}, \beta_{i}\right] \cdot u_{n+2} \cdots u_{1}\right\rangle .
$$

Let us maintain the notation introduced in the proof of Theorem 4.2 Elements of $\hat{\Pi}_{g, n}$ in the conjugacy class of $\beta_{g}$ are then in natural bijective correspondence with the set $\mathcal{N}_{\gamma}$ of nodes of $\tilde{C}_{\gamma}$.

For a given $f \in \check{\Gamma}_{\vec{\gamma}}<\check{\Gamma}_{g, n}$, there is a lift $\tilde{f} \in \operatorname{Aut}\left(\hat{\Pi}_{g, n}\right)$ such that $\tilde{f}\left(\beta_{g}\right)=\beta_{g}$. From Lemma 4.3 it follows that such a lift $\tilde{f}$ is uniquely determined by $f$, modulo inner automorphisms by powers of $\beta_{g}$. 
By the remarks made in the proof of Theorem 4.2 it is clear that the isomorphism between fibers $\tilde{f}: \widetilde{\mathscr{C}}_{x} \rightarrow \widetilde{\mathscr{C}}_{\tilde{f}(x)}$, induced by $\tilde{f}$, sends the irreducible component of $\tilde{\mathscr{C}}_{x}$ stabilized by the subgroup $\hat{\Pi}_{g-1, n+2}$ of $\hat{\Pi}_{g, n}$ to the irreducible component of $\widetilde{\mathscr{C}}_{\tilde{f}(x)}$ stabilized by the same subgroup, and hence induces an automorphism $\tilde{f}^{\prime}: \hat{\Pi}_{g-1, n+2} \stackrel{\sim}{\rightarrow} \hat{\Pi}_{g-1, n+2}$. We then let $\check{\rho}_{\gamma}(f)$ be the image of $\tilde{f}^{\prime}$ in $\operatorname{Out}\left(\hat{\Pi}_{g-1, n+2}\right)$.

In order to complete the proof of the lemma, it is now enough to show that the kernel of $\check{\rho}_{\gamma}$ is generated by the Dehn twist $\tau_{\gamma}$. As above, we just treat the case of a non-separating s.c.c. $\gamma$ on $S_{g, n}$.

Let us keep the above notation and moreover let $t:=\alpha_{g}$. We then get the standard presentation of $\Pi_{g, n}$ as an HNN extension of its free subgroup $\Pi_{g-1, n+2}$ :

$$
\Pi_{g, n}=\left\langle t, \Pi_{g-1, n+2} \mid t u_{n+1}^{-1} t^{-1}=u_{n+2}\right\rangle .
$$

In terms of graphs of groups, we are saying that $\Pi_{g, n}$ is naturally isomorphic to the fundamental group of the loop of groups having for vertex group $\Pi_{g-1, n+2}$ and for edge group the free cyclic group spanned by $t$.

For a given $f \in \check{\Gamma}_{\gamma}$, we have seen that it admits a lift $\tilde{f}$ to an automorphism of $\hat{\Pi}_{g, n}$ which preserves the closed subgroup $\hat{\Pi}_{g-1, n+2}$, and $\check{\rho}_{\gamma}(f)$ is defined to be the outer automorphism induced by $\tilde{f}$ on this subgroup.

Let $f \in \check{\Gamma}_{\gamma}$ be such that $\check{\rho}_{\gamma}(f)=1$. It then admits a lift $\tilde{f} \in \operatorname{Aut}\left(\hat{\Pi}_{g, n}\right)$ which restricts to the identity on $\hat{\Pi}_{g-1, n+2}$. In particular, it holds that $\tilde{f}\left(u_{n+1}\right)=u_{n+1}$ and $\tilde{f}\left(u_{n+2}\right)=u_{n+2}$. The action of $\tilde{f}$ hence satisfies the condition:

$$
\tilde{f}\left(u_{n+2}\right)=\tilde{f}(t) u_{n+1}^{-1} \tilde{f}(t)^{-1}=u_{n+2}=t u_{n+1}^{-1} t^{-1} .
$$

By Lemma 4.3, this identity implies $\tilde{f}(t)=t u_{n+1}^{k}$, for some $k \in \hat{\mathbb{Z}}$. It then follows that $f$ equals the power $\tau_{\gamma}^{-k} \in \check{\Gamma}_{g, n}$, since the power $\tau_{\gamma}^{-k}$ also lifts to an automorphism $\tilde{\tau}_{\gamma}^{-k}$ of $\hat{\Pi}_{g, n}$ such that $\tilde{\tau}_{\gamma}^{-k}(t)=t u_{n+1}^{k}$ and which restricts to the identity on the subgroup $\hat{\Pi}_{g-1, n+2}$.

Remark 4.7. Theorem 4.2 and Theorem 4.5 yield, in particular, a description of the links of simplices in the complex of profinite curves $L\left(\hat{\Pi}_{g, n}\right)$ analogous to the description of links of simplices in the curve complex $C\left(S_{g, n}\right)$. Indeed, with the same notation of Theorem 4.5, the link $\operatorname{Lk}(\sigma)$ of the simplex $\sigma$ in the simplicial profinite complex $L\left(\hat{\Pi}_{g, n}\right)$ is naturally isomorphic to the join of simplicial profinite complexes:

$$
L\left(\hat{\Pi}_{g_{1}, n_{1}}\right) * \ldots * L\left(\hat{\Pi}_{g_{h}, n_{h}}\right) .
$$

\section{Profinite Dehn twists in $\check{\Gamma}_{g, n}$}

A basic feature of classical Teichmüller theory (cf. $\S 2,[5]$ ) is the fact that the set $\mathscr{L}$ of isotopy classes of non-peripheral s.c.c.'s on $S_{g, n}$ parametrizes the set of Dehn twists of $\Gamma_{g, n}$, which is the standard set of generators for this group. In other words, the assignment $\gamma \mapsto \tau_{\gamma}$ for $\gamma \in \mathscr{L}$ defines an embedding $d: \mathscr{L} \hookrightarrow \Gamma_{g, n}$ for $2 g-2+n>0$.

The set $\left\{\tau_{\gamma}\right\}_{\gamma \in \mathscr{L}}$ of all Dehn twists of $\Gamma_{g, n}$ is closed under conjugation and falls in a finite set of conjugacy classes which are in bijective correspondence with the possible topological types of the Riemann surface $S_{g, n} \backslash \gamma$. So, it is natural to define, for a given profinite completion $\Gamma_{g, n}^{\prime}$ of the Teichmüller group $\Gamma_{g, n}$, the set 
of profinite Dehn twists of $\Gamma_{g, n}^{\prime}$ to be the closure of the image of the set $\left\{\tau_{\gamma}\right\}_{\gamma \in \mathscr{L}}$ inside $\Gamma_{g, n}^{\prime}$, which is the same as the union of the conjugacy classes in $\Gamma_{g, n}^{\prime}$ of the images of the Dehn twists of $\Gamma_{g, n}$.

More generally, there is a natural $\Gamma_{g, n}$-equivariant map $d_{k}: \mathscr{L} \rightarrow \check{\Gamma}_{g, n}$, defined by the assignment $\gamma \mapsto \tau_{\gamma}^{k}$, where $\Gamma_{g, n}$ acts by conjugation on $\check{\Gamma}_{g, n}$. From the universal property of the $\check{\Gamma}_{g, n}$-completion and Theorem 4.2, it then follows that the map $d_{k}$ extends to a continuous $\check{\Gamma}_{g, n}$-equivariant map $\hat{d}_{k}: \hat{\mathscr{L}} \rightarrow \check{\Gamma}_{g, n}$, whose image is the set of $k$-th powers of profinite Dehn twists.

In particular, a profinite s.c.c. $\gamma \in \hat{\mathscr{L}}$ determines a profinite Dehn twist, which we denote by $\tau_{\gamma}$, in the procongruence Teichmüller group $\check{\Gamma}_{g, n}$. The following theorem says that this provides a parametrization of the set of profinite Dehn twists of $\check{\Gamma}_{g, n}$ :

Theorem 5.1. For $2 g-2+n>0$ and any $k \in \hat{\mathbb{Z}} \backslash 0$, there is a natural injective map $\hat{d}_{k}: \hat{\mathscr{L}} \hookrightarrow \check{\Gamma}_{g, n}$ which assigns to a profinite s.c.c. $\gamma \in \hat{\mathscr{L}}$ the $k$-th power of the profinite Dehn twist $\tau_{\gamma}$.

In order to prove Theorem 5.1, a long preliminary digression is necessary.

Definition 5.2. Let us assume that the base point of the fundamental group $\Pi_{g, n}$ of $S_{g, n}$ is the marked point $P_{n+1}$. Let $\mathscr{L}_{g, n}^{*}$ be the set of relative isotopy classes of $P_{n+1}$-pointed oriented non-peripheral s.c.c.'s on $S_{g, n}$. This set embeds in the fundamental group $\Pi_{g, n}$ (cf. Theorem 3.4.15 in [11]).

Let us then define the set of $P_{n+1}$-pointed profinite oriented non-peripheral s.c.c.' $s \hat{\mathscr{L}}_{g, n}^{*}$ to be the closure of $\mathscr{L}_{g, n}^{*}$ inside the profinite completion $\hat{\Pi}_{g, n}$. The quotients of the sets $\mathscr{L}_{g, n}^{*}$ and $\hat{\mathscr{L}}_{g, n}^{*}$ by the action of inner automorphisms are, respectively, the set of oriented non-peripheral s.c.c.'s $\mathscr{L}_{g, n}^{o r}$ and of profinite oriented non-peripheral s.c.c.'s $\hat{\mathscr{L}}_{g, n}^{\text {or }}$.

Remark 5.3. There is a natural continuous surjective map $\hat{q}: \hat{\mathscr{L}}_{g, n}^{*} \rightarrow \hat{\mathscr{L}}_{g, n}$. We then let $\gamma:=\hat{q}(\vec{\gamma})$ and say that $\vec{\gamma} \in \hat{\mathscr{L}}_{g, n}^{*}$ lifts the profinite s.c.c. $\gamma \in \hat{\mathscr{L}}_{g, n}$.

For $2 g-2+n>0$, there is a natural monomorphism $i: \Pi_{g, n} \hookrightarrow \Gamma_{g, n+1}$ which, for the relative isotopy class of a $P_{n+1}$-pointed oriented s.c.c. $\vec{\gamma}$, is described as follows. Let $U_{\gamma}$ be a closed tubular neighborhood of $\vec{\gamma}$. The boundary of $U_{\gamma}$ consists of two s.c.c.'s on $S_{g, n+1}$. Let us denote by $\gamma_{1}$ the boundary component of $U_{\gamma}$ which lies at the right of $\vec{\gamma}$, with respect to its orientation, and by $\gamma_{2}$ the one which lies at its left. The isotopy classes of the pair of s.c.c.'s $\left\{\gamma_{1}, \gamma_{2}\right\}$ only depend on the class $[\vec{\gamma}]$ of $\vec{\gamma}$ in $\Pi_{g, n}$. The monomorphism $i$ then assigns to the class of $\vec{\gamma}$ the product of Dehn twists $\tau_{\gamma_{1}} \cdot \tau_{\gamma_{2}}^{-1}$ (see $\S 3$ in [5] for more details on this construction).

It is easy to see that the assignment $[\vec{\gamma}] \mapsto\left[\gamma_{i}\right]$ is $\Gamma_{g, n+1}$-equivariant, for $i=1,2$, where $\Gamma_{g, n+1}$ acts on its subgroup $\Pi_{g, n}$ by conjugation. In particular, this defines two $\Gamma_{g, n+1}$-equivariant maps $s_{i}: \mathscr{L}_{g, n}^{*} \rightarrow \mathscr{L}_{g, n+1}$, for $i=1,2$, such that the product map $s_{1} \times s_{2}: \mathscr{L}_{g, n}^{*} \rightarrow \mathscr{L}_{g, n+1} \times \mathscr{L}_{g, n+1}$ is injective.

It is easy to check that the image of both maps $s_{1}$ and $s_{2}$ is the subset $\mathscr{L}_{g, n+1}^{b}$ of $\mathscr{L}_{g, n+1}$ consisting of non-peripheral s.c.c.'s which do not bound a two-punctured disc, with one of the two punctures labeled by $P_{n+1}$. Let us denote by $\hat{\mathscr{L}}_{g, n+1}^{b}$ 
the closure of $\mathscr{L}_{g, n+1}^{b}$ inside the profinite set $\hat{\mathscr{L}}_{g, n+1}$. The following is basically a reformulation of Proposition 2.7 of [8]:

Proposition 5.4. For $2 g-2+n>0$, the profinite set $\hat{\mathscr{L}}_{g, n}^{*}$ is the $\check{\Gamma}_{g, n+1}$-completion of $\mathscr{L}_{g, n}^{*}$. In particular, the maps $s_{i}$ extend naturally to $\check{\Gamma}_{g, n+1}$-equivariant surjective continuous maps $\hat{s}_{i}: \hat{\mathscr{L}}_{g, n}^{*} \rightarrow \hat{\mathscr{L}}_{g, n+1}^{b}$, for $i=1,2$, such that the product map $\hat{s}_{1} \times \hat{s}_{2}: \hat{\mathscr{L}}_{g, n}^{*} \rightarrow \hat{\mathscr{L}}_{g, n+1}^{b} \times \hat{\mathscr{L}}_{g, n+1}^{b}$ is injective.

Proof. In order to prove that $\hat{\mathscr{L}}_{g, n}^{*}$ is the $\check{\Gamma}_{g, n+1}$-completion $\check{\mathscr{L}}_{g, n}^{*}$ of $\mathscr{L}_{g, n}^{*}$, let us observe that there is a $\Gamma_{g, n}$-equivariant isomorphism: $\mathscr{L}_{g, n}^{o r} \cong \mathscr{L}_{g, n} \times\{ \pm 1\}$.

By Theorem 4.2, $\hat{\mathscr{L}}_{g, n}$ is the $\check{\Gamma}_{g, n}$-completion of $\mathscr{L}_{g, n}$. Hence, the above isomorphism induces an isomorphism of $\check{\Gamma}_{g, n}$-completions $\check{\mathscr{L}}_{g, n}^{\text {or }} \cong \hat{\mathscr{L}}_{g, n} \times\{ \pm 1\}$, and so it holds at least $\check{\mathscr{L}}_{g, n}^{\text {or }} \cong \hat{\mathscr{L}}_{g, n}^{\text {or }}$.

But now, by definition, $\mathscr{L}_{g, n}^{o r}=\mathscr{L}_{g, n}^{*} / \Pi_{g, n}$. Hence, $\check{\mathscr{L}}_{g, n} \cong \check{\mathscr{L}}_{g, n}^{*} / \hat{\Pi}_{g, n}$ holds. On the other hand, also by definition, $\hat{\mathscr{L}}_{g, n}^{o r}=\hat{\mathscr{L}}_{g, n}^{*} / \hat{\Pi}_{g, n}$. Therefore, $\hat{\mathscr{L}}_{g, n}^{*} / \hat{\Pi}_{g, n} \cong$ $\check{\mathscr{L}}_{g, n}^{*} / \hat{\Pi}_{g, n}$ holds, which implies that $\hat{\mathscr{L}}_{g, n}^{*}$ is the $\check{\Gamma}_{g, n+1}$-completion of $\mathscr{L}_{g, n}^{*}$.

By the universal property of the $\check{\Gamma}_{g, n+1}$-completion, the maps $s_{i}$ then extend to surjective continuous $\check{\Gamma}_{g, n+1}$-equivariant maps $\hat{s}_{i}: \hat{\mathscr{L}}_{g, n}^{*} \rightarrow \hat{\mathscr{L}}_{g, n+1}^{b}$, for $i=1,2$.

The product map $\hat{s}_{1} \times \hat{s}_{2}$ is injective, since it factors the injective map which assigns to $\vec{\gamma} \in \hat{\mathscr{L}}_{g, n}^{*}$ the product of profinite Dehn twists $\tau_{\hat{s}_{1}(\vec{\gamma})} \tau_{\hat{s}_{2}(\vec{\gamma})} \in \check{\Gamma}_{g, n+1}$.

Remark 5.5. For $2 g-2+n>0$ and $k \in \hat{\mathbb{Z}} \backslash 0$, the assignment $\vec{\gamma} \mapsto \tau_{\hat{s}_{1}(\vec{\gamma})}^{k} \tau_{\hat{s}_{2}(\vec{\gamma})}^{-k}$, for $\vec{\gamma} \in \hat{\mathscr{L}}_{g, n}^{*}$, defines a $\check{\Gamma}_{g, n+1}$-equivariant continuous injective map $\hat{\mathscr{L}}_{g, n}^{*} \hookrightarrow \check{\Gamma}_{g, n+1}$.

The injectivity of the map $\hat{s}_{1} \times \hat{s}_{2}$ then implies that the centralizer in $\check{\Gamma}_{g, n+1}$ of the product of powers of Dehn twists $\tau_{\hat{s}_{1}(\vec{\gamma})}^{k} \tau_{\hat{s}_{2}(\vec{\gamma})}^{-k}$ is the stabilizer $\check{\Gamma}_{\sigma}$ of the 1-simplex $\sigma:=\left\{\hat{s}_{1}(\vec{\gamma}), \hat{s}_{2}(\vec{\gamma})\right\} \in L\left(\hat{\Pi}_{g, n+1}\right)$.

In order to prove Theorem [5.1, it is necessary to describe the $\hat{\Pi}_{g, n}$-conjugacy classes of the powers of profinite Dehn twists in $\check{\Gamma}_{g, n+1}$. Let $\check{p}: \check{\Gamma}_{g, n+1} \rightarrow \check{\Gamma}_{g, n}$ be the epimorphism induced by filling in the $(n+1)$-th puncture on $S_{g, n+1}$, whose kernel is identified with $\hat{\Pi}_{g, n}$.

Theorem 5.6. For $2 g-2+n>0$, let there be given $\gamma \in \hat{\mathscr{L}}_{g, n}$ and $k \in \hat{\mathbb{Z}} \backslash\{0\}$. Let $\vec{\gamma} \in \hat{\mathscr{L}}_{g, n}^{*}$ be a pointed oriented profinite s.c.c. which lifts $\gamma$. The set of powers of Dehn twists in $\check{\Gamma}_{g, n+1}$ which lift $\tau_{\gamma}^{k} \in \check{\Gamma}_{g, n}$ along the epimorphism $\check{p}$ : $\check{\Gamma}_{g, n+1} \rightarrow \check{\Gamma}_{g, n}$ is the union of the $\hat{\Pi}_{g, n}$-conjugacy orbits of $\tau_{\hat{s}_{1}(\vec{\gamma})}^{k}$ and $\tau_{\hat{s}_{2}(\vec{\gamma})}^{k}$. These orbits are distinct when $\gamma$ is separating and coincide when $\gamma$ is non-separating.

Remark 5.7. Let us observe that in the discrete case the analogue of Theorem 5.6 follows from elementary topological considerations. Indeed, lifting the power $\tau_{\gamma}^{k} \in$ $\Gamma_{g, n}$ of a Dehn twist along the epimorphism $\Gamma_{g, n+1} \rightarrow \Gamma_{g, n}$, induced by filling in the $(n+1)$-th puncture on $S_{g, n+1}$, amounts to removing a point from the Riemann surface $S_{g, n}$ outside the curve $\gamma$ and then moving the puncture to the marked point $P_{n+1}$. So, modulo point-pushing homeomorphisms based at $P_{n+1}$, which correspond to the conjugacy action of $\Pi_{g, n}$, there is essentially one choice in case $\gamma$ is non-separating and two in case $\gamma$ is separating. 
It is enough to prove Theorem 5.6 for a discrete s.c.c. $\gamma \in \mathscr{L}_{g, n}$ and for $k \in$ $\mathbb{Z} \backslash\{0\}$. In order to do this, we are going to use the short exact sequence (2.1) of Section 2

Let $K$ be a finite index characteristic proper subgroup of $\Pi_{g, n}$ and let $\Gamma_{g,[n]+1}$ be the group of homotopy classes of orientation preserving homeomorphisms of $S_{g, n}$ which fix the base point $P_{n+1}$ of the fundamental group $\Pi_{g, n}$. Since $K$ is a characteristic subgroup of $\Pi_{g, n}$, it is induced by a representation $\Gamma_{g,[n]+1} \rightarrow$ Out $(K)$, such that the image of the subgroup $\Pi_{g, n}$ of $\Gamma_{g,[n]+1}$ is identified, by the Nielsen realization theorem, with the covering transformation group $G_{K}$ of the covering $p_{K}: S_{K} \rightarrow S_{g, n}$ associated to the subgroup $K$.

By the Nielsen realization theorem, with the notation of Section 2, there is then a commutative diagram with exact rows and surjective vertical maps:

$$
\begin{aligned}
& \begin{array}{ccc}
1 \rightarrow & \prod_{g, n} \rightarrow & \Gamma_{g,[n]+1} \\
\downarrow & \downarrow & \Gamma_{g,[n]} \rightarrow 1
\end{array} \\
& 1 \rightarrow G_{K} \rightarrow N_{\Gamma\left(S_{K}\right)}\left(G_{K}\right) \rightarrow \Gamma_{g,[n]} \rightarrow 1 .
\end{aligned}
$$

Definition 5.8. An element of the group $N_{\Gamma\left(S_{K}\right)}\left(G_{K}\right)$ is called a pseudo-twist if it is the image of a Dehn twist of $\Gamma_{g,[n]+1}$ via the epimorphism $\Gamma_{g,[n]+1} \rightarrow N_{\Gamma\left(S_{K}\right)}\left(G_{K}\right)$ of diagram (5.1).

Let us observe that, for any s.c.c. $\gamma$ on $S_{g, n}$, the surface $S_{K} \backslash p_{K}^{-1}(\gamma)$ is disconnected and such that every circle in $p_{K}^{-1}(\gamma)$ is a boundary component of two distinct connected components (cf. the proof of Lemma 3.10 of [9]). Then, the following holds:

Proposition 5.9. $\quad$ i) An element $v_{\gamma} \in N_{\Gamma\left(S_{K}\right)}\left(G_{K}\right)$ which lifts the $k$-th power of a Dehn twist $\tau_{\gamma}^{k} \in \Gamma_{g,[n]}$, for $k \in \mathbb{Z} \backslash\{0\}$, is the $k$-th power of a pseudo-twist if and only if it restricts to the identity on at least one of the components of the surface $S_{K} \backslash p_{K}^{-1}(\gamma)$.

ii) Let $S$ and $S^{\prime}$ be the two connected components of the surface $S_{K} \backslash p_{K}^{-1}(\gamma)$ sharing the boundary component $\tilde{\gamma} \subset p_{K}^{-1}(\gamma)$. Then let $v_{\tilde{\gamma}}$ and $v_{\tilde{\gamma}}^{\prime}$ be the $k$-th powers of pseudo-twists which lift the given power of Dehn twist $\tau_{\gamma}^{k}$ and which restrict to the identity, respectively, on $S$ and $S^{\prime}$. Then, there is an element $\gamma_{*} \in \Pi_{g, n}$, whose free homotopy class contains $\gamma$, such that its image $\bar{\gamma}_{*}$ in $G_{K}$ generates the stabilizer of the s.c.c. $\tilde{\gamma} \subset S_{K}$ and $v_{\tilde{\gamma}}^{\prime} v_{\tilde{\gamma}}^{-1}=\bar{\gamma}_{*}^{k}$ holds.

Proof. Let us assume that $\gamma$ is non-separating; the other case can be treated similarly. With the same notation and conventions of the proof of Lemma 4.6. let us assume moreover that $\gamma$ is disjoint from the loops $\alpha_{1}, \ldots, \alpha_{g-1}, \beta_{1}, \ldots, \beta_{g-1}$, $u_{1}, \ldots, u_{n+2}$. The natural representation $\Gamma_{g,[n]+1} \hookrightarrow \operatorname{Aut}\left(\Pi_{g, n}\right)$ is then such that the power of Dehn twist $\tilde{\tau}_{\gamma}^{k} \in \Gamma_{g,[n]+1}$, which lifts the power of Dehn twist $\tau_{\gamma}^{k} \in$ $\Gamma_{g,[n]}$, acts trivially on the subgroup $\Pi_{g-1, n+2}$ of $\Pi_{g, n}$. In particular, $\tilde{\tau}_{\gamma}^{k}$ acts trivially on the intersection $K \cap \Pi_{g-1, n+2}$.

Let $\tilde{P}$ be a point of $S_{K}$ lying above the base point $P_{n+1}$ of the fundamental group $\Pi_{g, n}$ of $S_{g, n}$ and let us identify $K$ with the fundamental group of $S_{K}$ based at $\tilde{P}$. The fundamental group of the connected component of $S_{K} \backslash p_{K}^{-1}(\gamma)$ which contains the point $\tilde{P}$ clearly identifies with the intersection $K \cap \Pi_{g-1, n+2}$. Hence, the power of Dehn twist $\tilde{\tau}_{\gamma}^{k}$ induces the trivial action on this connected component. 
Since all connected components of $S_{K} \backslash p_{K}^{-1}(\gamma)$ are in a single orbit for the action of $G_{K}$, part $i$ ) of Proposition 5.9 follows.

As for part $i i$ ), let us just observe that $v_{\tilde{\gamma}}^{\prime} v_{\tilde{\gamma}}^{-1}$ is the image, via the representation $\Gamma_{g,[n]+1} \rightarrow N_{\Gamma\left(S_{K}\right)}\left(G_{K}\right)$, of the $k$-th power of the bounding pair map associated to an element $\gamma_{*} \in \Pi_{g, n}$ which identifies inside the group $\operatorname{Out}(K)$ with the element of $G_{K}$ with the properties stated in part $\left.i i\right)$.

Let $\check{N}_{\Gamma\left(S_{K}\right)}\left(G_{K}\right)$ be the closure of $N_{\Gamma\left(S_{K}\right)}\left(G_{K}\right)$ inside the profinite group $\check{\Gamma}\left(S_{K}\right)$. This is the same profinite completion as that induced by the procongruence completion $\check{\Gamma}_{g,[n]+1}$ via the natural epimorphism $\Gamma_{g,[n]+1} \rightarrow N_{\Gamma\left(S_{K}\right)}\left(G_{K}\right)$. Hence, the diagram (5.1) induces a commutative diagram with exact rows and surjective vertical maps:

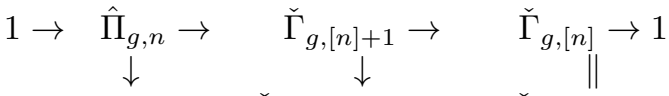

$$
\begin{aligned}
& 1 \rightarrow \quad G_{K} \rightarrow \quad \check{N}_{\Gamma\left(S_{K}\right)}\left(G_{K}\right) \rightarrow \check{\Gamma}_{g,[n]} \rightarrow 1 \text {. }
\end{aligned}
$$

Moreover, the upper row of the diagram is isomorphic to the inverse limit of the bottom rows when $K$ varies among all finite index characteristic subgroups of $\Pi_{g, n}$. Theorem 5.6 then follows from the lemma:

Lemma 5.10. Let $\tau_{\gamma}^{k} \in \check{\Gamma}_{g,[n]}$, for $k \in \mathbb{Z} \backslash\{0\}$, be the power of a discrete Dehn twist and let $v_{\gamma}$ be a lift of this element to $\check{N}_{\Gamma\left(S_{K}\right)}\left(G_{K}\right)$, which then belongs to the discrete subgroup $N_{\Gamma\left(S_{K}\right)}\left(G_{K}\right)$. Then, $v_{\gamma}$ belongs to the closure of the set of $k$-th powers of pseudo-twists in the profinite group $\check{N}_{\Gamma\left(S_{K}\right)}\left(G_{K}\right)$ if and only if it is the $k$-th power of a pseudo-twist.

Proof. The inverse image of $\Gamma_{g,[n]}$, considered as a subgroup of $\check{\Gamma}_{g,[n]}$, via the epimorphism $\check{N}_{\Gamma\left(S_{K}\right)}\left(G_{K}\right) \rightarrow \check{\Gamma}_{g,[n]}$ is the discrete subgroup $N_{\Gamma\left(S_{K}\right)}\left(G_{K}\right)$. Therefore, any lift in the profinite group $\check{N}_{\Gamma\left(S_{K}\right)}\left(G_{K}\right)$ of a discrete element of the profinite group $\check{\Gamma}_{g,[n]}$ is also discrete.

Let $v_{\gamma} \in N_{\Gamma\left(S_{K}\right)}\left(G_{K}\right)$ be a lift of $\tau_{\gamma}^{k} \in \Gamma_{g,[n]}$ which does not restrict to the identity on any component of the surface $S_{K} \backslash p_{K}^{-1}(\gamma)$. By Proposition 5.9, in order to prove the lemma, it is enough to show that $v_{\gamma}$ is not in the closure in $\check{N}_{\Gamma\left(S_{K}\right)}\left(G_{K}\right)$ of the set of $k$-th powers of pseudo-twists of $N_{\Gamma\left(S_{K}\right)}\left(G_{K}\right)$. Let us then assume, on the contrary, that $v_{\gamma}$ belongs to such a closure and let us show how this leads to a contradiction.

Let $s$ be the smallest positive integer such that $\gamma^{s} \in K$. Then, $\tau_{\gamma}^{s} \in \Gamma_{g,[n]}$ lifts, canonically, in $N_{\Gamma\left(S_{K}\right)}\left(G_{K}\right)$ to the product, which we denote by $\xi_{\gamma}$, of the Dehn twists about the s.c.c.'s contained in $p_{K}^{-1}(\gamma)$. This element is in the centralizer of the finite group $G_{K}$. It follows that the $\tilde{N}_{\Gamma\left(S_{K}\right)}\left(G_{K}\right)$-conjugacy orbit of $\xi_{\gamma}^{k}$ is mapped bijectively, by the epimorphism $\check{N}_{\Gamma\left(S_{K}\right)}\left(G_{K}\right) \rightarrow \check{\Gamma}_{g,[n]}$, onto the $\check{\Gamma}_{g,[n]}$-conjugacy orbit of $\tau_{\gamma}^{s k}$.

By the above assumption, $v_{\gamma}^{s}$ is in the closure in $\check{N}_{\Gamma\left(S_{K}\right)}\left(G_{K}\right)$ of the set of $s k$-th powers of pseudo-twists of $N_{\Gamma\left(S_{K}\right)}\left(G_{K}\right)$. Hence, $v_{\gamma}^{s}$ is in the $\check{N}_{\Gamma\left(S_{K}\right)}\left(G_{K}\right)$-conjugacy orbit of $\xi_{\gamma}^{k}$. Since both elements project to $\tau_{\gamma}^{s k}$, the identity $v_{\gamma}^{s}=\xi_{\gamma}^{k}$ holds.

For a given commutative ring $A$ with 1 , let us denote the homology group $H_{1}\left(\bar{S}_{K}, A\right)$ simply by $H_{A}$ and by $\left\langle_{-,}\right\rangle_{A}$ its standard symplectic form. Then let 
$\mathfrak{s p}\left(H_{A}\right)$ be the Lie algebra of the symplectic group $\operatorname{Sp}\left(H_{A}\right)$, i.e. the $A$-module of endomorphisms $\varphi$ of $H_{A}$ satisfying the identity $\langle\varphi(x), y\rangle_{A}+\langle x, \varphi(y)\rangle_{A}=0$, for all $x, y \in H_{A}$.

For $\varphi \in \mathfrak{s p}\left(H_{A}\right)$, the assignment $\varphi \mapsto\left\langle\left\langle_{-}, \varphi\left({ }_{-}\right)\right\rangle_{A}\right.$ defines a natural isomorphism:

$$
\mathfrak{s p}\left(H_{A}\right) \stackrel{\sim}{\rightarrow} \mathrm{L}_{s}^{2}\left(H_{A}\right),
$$

where $\mathrm{L}_{s}^{2}\left(H_{A}\right)$ is the $A$-module of symmetric bilinear forms on $H_{A}$. There is also a natural isomorphism $\mathrm{L}_{s}^{2}\left(H_{A}\right) \cong \operatorname{Sym}^{2} H_{A}^{*}$, where $H_{A}^{*}:=\operatorname{Hom}_{A}\left(H_{A}, A\right)$ is the dual $A$-module which can be identified with the cohomology group $H^{1}\left(\bar{S}_{K}, A\right)$.

The Poincaré dual of an element $a \in H_{A}$ is the $A$-linear map $a^{\vee}:=\left\langle{ }_{-}, a\right\rangle_{A} \in H_{A}^{*}$ and, for a submodule $M$ of $H_{A}$, its Poincaré dual $M^{\vee}$ is defined to be the submodule $\left\{m^{\vee} \mid m \in M\right\}$ of $H_{A}^{*}$. In contrast with $\left({ }_{-}\right)^{*}$, this is a covariant functor.

Let us also denote by $\left\langle_{-},{ }_{-}\right\rangle_{A}$ the symplectic form induced on $H_{A}^{*}$ by the standard symplectic form on $H_{A}$, i.e. $\left\langle a^{\vee}, b^{\vee}\right\rangle_{A}:=\langle b, a\rangle_{A}$. The Poincaré duals of elements and submodules of $H_{A}^{*}$ are defined as above. For $a \in H_{A}$ or $a \in H_{A}^{*}$, the identity $\left(a^{\vee}\right)^{\vee}=a$ then holds, where $H_{A}$ is identified with $H_{A}^{* *}$ by the usual canonical assignment $a \mapsto[f \mapsto f(a)]$.

An element $f \in \Gamma\left(S_{K}\right)$ induces a symplectic automorphism of $H_{A}$, which we also denote by $f$. Let us define the $\operatorname{logarithm} \log f$ to be the symmetric bilinear form on $H_{A}$ associated to the endomorphism $f-\mathrm{id}_{H_{A}}$.

For $\alpha$ an s.c.c. on the closed Riemann surface $\bar{S}_{K}$, let us denote by $\vec{\alpha}$ the cycle determined in $H_{A}$ by the s.c.c. $\alpha$ with a given orientation. From simple direct computations (cf. $\S 1$ in [16]), it follows that, for the Dehn twist $\tau_{\alpha}$, the identity $\log \tau_{\alpha}=\vec{\alpha}^{\vee} \otimes \vec{\alpha}^{\vee}$ holds. In particular, the logarithm of $\tau_{\alpha}$ does not depend on the chosen orientation on $\alpha$.

It is possible to recover the submodule $\langle\vec{\alpha}\rangle$ of $H_{A}$ generated by the cycle $\vec{\alpha}$ from the logarithm $\vec{\alpha}^{\vee} \otimes \vec{\alpha}^{\vee}$ of the Dehn twist $\tau_{\alpha}$ as the Poincaré dual of the dual of the cokernel of the corresponding symmetric bilinear form:

$$
\langle\vec{\alpha}\rangle=\left(\left(H_{A} / \operatorname{ker} \vec{\alpha}^{\vee} \otimes \vec{\alpha}^{\vee}\right)^{*}\right)^{\vee} .
$$

For a given symmetric bilinear form $\varphi \in \mathrm{Sym}^{2} H_{A}^{*}$, in order to simplify the terminology, we call the Poincaré dual of the dual of its cokernel, which is a submodule of $H_{A}$, the core of the form $\phi$. Thus, we can write core $\left(\vec{\alpha}^{\vee} \otimes \vec{\alpha}^{\vee}\right)=\langle\vec{\alpha}\rangle$.

More generally, for $A=\mathbb{Z}$, the following holds:

Lemma 5.11. For $c_{1}, \ldots, c_{h} \in \mathbb{Z}^{+}$and $\alpha_{1}, \ldots \alpha_{h} \in H_{\mathbb{Z}}$, the following holds:

$$
\left\langle\overrightarrow{\alpha_{1}}, \ldots, \vec{\alpha}_{h}\right\rangle=\text { core }\left(\sum_{i=1}^{h} c_{i}{\overrightarrow{\alpha_{i}}}^{\vee} \otimes{\overrightarrow{\alpha_{i}}}^{\vee}\right)
$$

Proof. The lemma follows from the identity

$$
\operatorname{ker}\left(\sum_{i=1}^{h} c_{i}{\overrightarrow{\alpha_{i}}}^{\vee} \otimes{\overrightarrow{\alpha_{i}}}^{\vee}\right)=\bigcap_{i=1}^{h} \operatorname{ker}{\overrightarrow{\alpha_{i}}}^{\vee} \otimes{\overrightarrow{\alpha_{i}}}^{\vee}
$$

which we are going to prove below, and the series of identities:

$$
\left(\left(H_{\mathbb{Z}} / \bigcap_{i=1}^{h} \operatorname{ker}{\overrightarrow{\alpha_{i}}}^{\vee} \otimes{\overrightarrow{\alpha_{i}}}^{\vee}\right)^{*}\right)^{\vee}=\left\langle\bigcup_{i=1}^{h}\left(H_{\mathbb{Z}} / \operatorname{ker}{\overrightarrow{\alpha_{i}}}^{\vee} \otimes{\overrightarrow{\alpha_{i}}}^{\vee}\right)^{*}\right\rangle^{\vee}=\left\langle\overrightarrow{\alpha_{1}}, \ldots, \overrightarrow{\alpha_{h}}\right\rangle \text {. }
$$


Let $\varphi:=\sum_{i=1}^{h} c_{i}{\overrightarrow{\alpha_{i}}}^{\vee} \otimes{\overrightarrow{\alpha_{i}}}^{\vee}$. Then, the symmetric bilinear form $\varphi$ induces a positive definite form on the quotient $H_{\mathbb{Z}} / \operatorname{ker} \varphi$. Since $\operatorname{ker} \varphi$ is a primitive sublattice of $H_{\mathbb{Z}}$, there is a splitting $H_{\mathbb{Z}}=\operatorname{ker} \varphi \oplus M$ such that $\varphi$ restricts to a positive definite symmetric bilinear form on $M$. It holds that $H_{\mathbb{Q}} \cong(\operatorname{ker} \varphi \otimes \mathbb{Q}) \oplus(M \otimes \mathbb{Q})$ and $\varphi$ extends to a symmetric bilinear form $\varphi^{\prime}$ on this $\mathbb{Q}$-vector space which restricts to a positive definite form on $M \otimes \mathbb{Q}$ and such that $\operatorname{ker} \varphi^{\prime}=\operatorname{ker} \varphi \otimes \mathbb{Q}$ and $\operatorname{ker} \varphi=$ $\operatorname{ker} \varphi^{\prime} \cap H_{\mathbb{Z}}$.

Let us denote by $\xi_{i}$ the extension of the symmetric bilinear form ${\overrightarrow{\alpha_{i}}}^{\vee} \otimes{\overrightarrow{\alpha_{i}}}^{\vee}$ to $H_{\mathbb{Q}}$, for $i=1, \ldots, h$. Then, it holds that $\varphi^{\prime}=\sum_{i=1}^{h} c_{i} \xi_{i}$ and $\operatorname{ker}{\overrightarrow{\alpha_{i}}}^{\vee} \otimes{\overrightarrow{\alpha_{i}}}^{\vee}=\operatorname{ker} \xi_{i} \cap H_{\mathbb{Z}}$, for $i=1, \ldots, h$. By elementary linear algebra, $\operatorname{ker} \varphi^{\prime}=\bigcap_{i=1}^{h} \operatorname{ker} \xi_{i}$, holds, and this implies the identity claimed above.

Let us denote by $\left(\log \xi_{\gamma}^{k}\right)_{p}$ the reduction $\bmod p$ of the symmetric bilinear form $\log \xi_{\gamma}^{k}$. The latter induces a non-degenerate form on the quotient $H_{\mathbb{Z}} / \operatorname{ker}\left(\log \xi_{\gamma}^{k}\right)$. Therefore, there is a non-empty open set $U$ of $\operatorname{Spec}(\mathbb{Z})$ such that, for all $p \in U$, the symmetric bilinear form $\left(\log \xi_{\gamma}^{k}\right)_{p}$ induces a non-degenerate form on the $\bmod p$ reduction $\left(H_{\mathbb{Z}} / \operatorname{ker}\left(\log \xi_{\gamma}^{k}\right)\right) \otimes \mathbb{F}_{p}$, which is then isomorphic to $H_{\mathbb{F}_{p}} / \operatorname{ker}\left(\log \xi_{\gamma}^{k}\right)_{p}$. It follows that $\left.\operatorname{ker}\left(\log \xi_{\gamma}^{k}\right)_{p} \cong \operatorname{ker}\left(\log \xi_{\gamma}^{k}\right)\right) \otimes \mathbb{F}_{p}$ holds for all primes $p \in U$.

For any given s.c.c. $\alpha$ on $S_{g, n}$, let us denote by $H_{\alpha}$ the primitive submodule of $H_{\mathbb{Z}}$ generated by the cycles supported in $p_{K}^{-1}(\alpha)$. For a prime $p$, let $\mathbb{F}_{p}$ be the field with $p$ elements and then let $H_{\alpha, p}$ be the image of $H_{\alpha}$ in the $\mathbb{F}_{p}$-vector space $H_{\mathbb{F}_{p}}$ via the natural epimorphism $H_{\mathbb{Z}} \rightarrow H_{\mathbb{F}_{p}}$. There is a natural isomorphism $H_{\alpha, p} \cong H_{\alpha} \otimes \mathbb{F}_{p}$.

By Lemma 5.11, $H_{\gamma}$ is the core of the bilinear form $\log \xi_{\gamma}^{k}=k \log \xi_{\gamma}$ on $H_{\mathbb{Z}}$. It follows that, for all primes $p \in U$, the core of the $\bmod p$ reduction $\left(\log v_{\gamma}^{s}\right)_{p}$ of the bilinear form $\log v_{\gamma}^{s}=\log \xi_{\gamma}^{k}$ is the subspace $H_{\gamma, p}$ of $H_{\mathbb{F}_{p}}$.

By hypothesis, there is a pseudo-twist $\tilde{\tau}_{\delta}$ of $N_{\Gamma\left(S_{K}\right)}\left(G_{K}\right)$, lifting a Dehn twist $\tau_{\delta} \in \Gamma_{g,[n]}$, such that $\tilde{\tau}_{\delta}^{k}$ and $v_{\gamma}$ map to the same element of the symplectic group $\operatorname{Sp}\left(H_{\mathbb{F}_{p}}\right)$, for some $p \in U$ with $p>2$. In particular, the core of the bilinear form $\left(\log \tilde{\tau}_{\delta}^{k s}\right)_{p}$ on $H_{\mathbb{F}_{p}}$ is the subspace $H_{\gamma, p}$. Since $H_{\delta, p}$ is contained in the core of $\left(\log \tilde{\tau}_{\delta}^{k s}\right)_{p}$ and is of the same dimension, i.e. the dimension of $H_{\gamma, p}$, it follows that $H_{\delta, p}=\operatorname{ker}\left(\log \tilde{\tau}_{\delta}^{k s}\right)_{p}=H_{\gamma, p}$ holds.

For a given s.c.c. $\alpha$ on $S_{g, n}$, let $\Sigma_{\alpha}$ be the dual graph of the degeneration of the Riemann surface $\bar{S}_{K}$ obtained by contracting all s.c.c.'s contained in $p_{K}^{-1}(\alpha)$. There is then an epimorphism $\Phi_{\alpha}: H_{\mathbb{Z}} \rightarrow H_{1}\left(\Sigma_{\alpha}, \mathbb{Z}\right)$ which is the Poincaré dual of the dual of the inclusion $H_{\alpha} \subset H_{\mathbb{Z}}$ (cf. Proposition 1 and 1bis in [10]). The epimorphism $\Phi_{\alpha}$ contains $H_{\alpha}$ in its kernel and induces an epimorphism $\bar{\Phi}_{\alpha}: H_{\mathbb{Z}} / H_{\alpha} \rightarrow H_{1}\left(\Sigma_{\alpha}, \mathbb{Z}\right)$. The standard symplectic form on $H_{\mathbb{Z}}$ induces a non-degenerate symplectic form on $K_{\alpha}:=\operatorname{ker} \bar{\Phi}_{\alpha}$.

The above statements hold for any system of coefficients for the homology. For a prime $p$, let $\bar{\Phi}_{\alpha, p}: H_{\mathbb{F}_{p}} / H_{\alpha, p} \rightarrow H_{1}\left(\Sigma_{\alpha}, \mathbb{F}_{p}\right)$ be the corresponding epimorphism with $\mathbb{F}_{p}$-coefficients and let $K_{\alpha, p}:=\operatorname{ker} \bar{\Phi}_{\alpha, p}$.

With the above notation, $K_{\gamma, p}=K_{\delta, p}$ holds and there is then a natural symplectic isomorphism $K_{\gamma} \otimes \mathbb{F}_{p} \cong K_{\delta} \otimes \mathbb{F}_{p}$. This isomorphism lifts to a symplectic isomorphism $K_{\gamma} \cong K_{\delta}$. If we identify the latter two $\mathbb{Z}$-modules by means of this isomorphism, the elements $v_{\gamma}$ and $\tilde{\tau}_{\delta}^{k}$ both act on the $\mathbb{Z}$-module $K_{\gamma}$ and have the same reduction modulo $p$. 
Let $\bar{S}_{\gamma}$ be the disconnected closed Riemann surface obtained by filling in all the punctures of $S_{K} \backslash p_{K}^{-1}(\gamma)$. Then, $H_{1}\left(\bar{S}_{\gamma}, \mathbb{Z}\right)=K_{\gamma}$ and the lift $v_{\gamma}$ acts on $\bar{S}_{\gamma}$ as a finite order homeomorphism which does not restrict to the identity on any component of $\bar{S}_{\gamma}$.

Then let $\bar{S}_{\gamma}^{\prime}$ be the quotient of $\bar{S}_{\gamma}$ by the action of the finite cyclic group generated by $v_{\gamma}$. It follows that the invariant submodule $\left(K_{\gamma}\right)^{v_{\gamma}}$ is naturally isomorphic to the homology group $H_{1}\left(\bar{S}_{\gamma}^{\prime}, \mathbb{Z}\right)$ and hence that $\left(K_{\gamma}\right)^{v_{\gamma}}$ does not contain any primitive non-degenerate symplectic submodule of $K_{\gamma}$.

By Proposition 5.9, the action induced by $\tilde{\tau}_{\delta}^{k}$ on $\bar{S}_{\delta}$ restricts to the identity on at least a component of this surface and then preserves a primitive non-degenerate symplectic submodule of $K_{\delta}$. Therefore, the action induced by $\tilde{\tau}_{\delta}^{k}$ on $K_{\gamma}$, by means of the above identification, is not conjugated to that of $v_{\gamma}$ but induces the same action on $K_{\gamma} \otimes \mathbb{F}_{p}$. Lemma 5.10 is then a consequence of the following lemma which gives a contradiction:

Lemma 5.12. Let $C_{1}$ and $C_{2}$ two finite cyclic subgroups of $\mathrm{Sp}_{2 g}(\mathbb{Z})$ which are not conjugated inside this group. Then, for all integers $m \geq 3$, their images $\bar{C}_{1}$ and $\bar{C}_{2}$ in the quotient group $\mathrm{Sp}_{2 g}(\mathbb{Z} / m)$ are also non-conjugated.

Proof. This fact is possibly well known but is, at least, an easy consequence of the theory of abelian varieties with automorphisms. Let $\mathcal{A}_{1}$ and $\mathcal{A}_{2}$ be the irreducible substacks of $\mathcal{A}_{g}$, the moduli stack of complex principally polarized abelian varieties of dimension $g$, parametrizing abelian varieties with an automorphism conjugated, respectively, to $C_{1}$ and $C_{2}$. Since $C_{1}$ and $C_{2}$ are not conjugated in $\operatorname{Sp}_{2 g}(\mathbb{Z})$, it follows that $\mathcal{A}_{1} \neq \mathcal{A}_{2}$.

Let $\mathcal{A}_{g}^{(m)}$ be the moduli stack of complex principally polarized abelian varieties of dimension $g$ with a level $m \geq 3$ structure. The natural morphism $p: \mathcal{A}_{g}^{(m)} \rightarrow \mathcal{A}_{g}$ is a Galois covering with deck transformation group $\operatorname{Sp}_{2 g}(\mathbb{Z} / m)$ and the irreducible components of $p^{-1}\left(\mathcal{A}_{1}\right)$ and $p^{-1}\left(\mathcal{A}_{2}\right)$ are the fixed point loci of the conjugates inside $\mathrm{Sp}_{2 g}(\mathbb{Z} / m)$ of the subgroups $\bar{C}_{1}$ and $\bar{C}_{2}$, respectively. If these two groups were in the same conjugacy class, it would follow that $\mathcal{A}_{1}=\mathcal{A}_{2}$, which is not the case.

By Proposition 5.4 there are continuous maps $\hat{s}_{i *}^{k}: \hat{\mathscr{L}}_{g, n}^{*} \rightarrow \check{\Gamma}_{g, n+1}$, for $i=1,2$ and $k \in \hat{\mathbb{Z}}$, which send a profinite loop $\vec{\gamma} \in \hat{\mathscr{L}}_{g, n}^{*}$ to the power of Dehn twist $\tau_{\hat{s}_{i}(\vec{\gamma})}^{k} \in \check{\Gamma}_{g, n+1}$.

For $\gamma \in \hat{\mathscr{L}}_{g, n}$ a given profinite s.c.c., let us then describe in a more precise way the set of all pairs of $k$-th powers of Dehn twists $\left(\tau_{\hat{s}_{1}(\vec{\alpha})}^{k}, \tau_{\hat{s}_{2}(\vec{\alpha})}^{k}\right)$ for $\vec{\alpha} \in \mathscr{L}_{g, n}^{*}$ such that $\tau_{\hat{q}(\vec{\alpha})}^{k}=\tau_{\gamma}^{k}$, where $\hat{q}: \hat{\mathscr{L}}_{g, n}^{*} \rightarrow \hat{\mathscr{L}}_{g, n}$ is the natural continuous surjective map.

Let us define the $\check{\Gamma}_{g, n+1}$-equivariant continuous map $\hat{s}_{*}^{k}: \hat{\mathscr{L}}_{g, n}^{*} \rightarrow \check{\Gamma}_{g, n+1} \times \check{\Gamma}_{g, n+1}$ which assigns to a profinite oriented pointed s.c.c. $\vec{\gamma} \in \hat{\mathscr{L}}_{g, n}^{*}$ the pair of powers of profinite Dehn twists $\left(\tau_{\hat{s}_{1}(\vec{\gamma})}^{k}, \tau_{\hat{s}_{2}(\vec{\gamma})}^{k}\right)$, where $\check{\Gamma}_{g, n+1}$ act on the product $\check{\Gamma}_{g, n+1} \times \check{\Gamma}_{g, n+1}$ by conjugation via the diagonal embedding. The map $\hat{s}_{*}^{k}$ is injective, since it factors the injective map which sends $\vec{\gamma} \in \hat{\mathscr{L}}_{g, n}^{*}$ to the product $\tau_{\hat{s}_{1}(\vec{\gamma})}^{k} \tau_{\hat{s}_{2}(\vec{\gamma})}^{-k} \in \check{\Gamma}_{g, n+1}$.

Theorem 5.13. For $2 g-2+n>0$ and $k \in \hat{\mathbb{Z}} \backslash\{0\}$, let $\gamma \in \hat{\mathscr{L}}_{g, n}$ be a given profinite s.c.c. Then, the set formed by the ordered pairs $\left(\tau_{\hat{s}_{1}(\vec{\alpha})}^{k}, \tau_{\hat{s}_{2}(\vec{\alpha})}^{k}\right)$, for $\vec{\alpha} \in \hat{\mathscr{L}}_{g, n}^{*}$, such 
that $\tau_{\hat{q}(\vec{\alpha})}^{k}=\tau_{\gamma}^{k}$, consists of two $\hat{\Pi}_{g, n}$-orbits, each corresponding to an orientation of $\gamma$, where the group $\hat{\Pi}_{g, n}$ acts diagonally on such pairs by conjugation.

Proof. As usual, it is enough to prove the theorem in case $\gamma$ is actually the class of an s.c.c. on the Riemann surface $S_{g, n}$.

By Theorem 5.6. the set of pairs of powers of profinite Dehn twists of the form $\left(\tau_{\hat{s}_{1}(\vec{\alpha})}^{k}, \tau_{\hat{s}_{2}(\vec{\alpha})}^{k}\right)$, for $\vec{\alpha} \in \hat{\mathscr{L}}_{g, n}^{*}$, lying above the element $\tau_{\gamma}^{k} \in \Gamma_{g, n}$, is the inverse limit of the set of pairs of powers of pseudo-twists described in ii) of Proposition 5.9. These pairs fit at most in two conjugacy classes, corresponding to the possible orientations of $\gamma$. Hence, the same holds for the pairs of powers of Dehn twists lying above $\tau_{\gamma}^{k}$.

On the other hand, since the two lifts $\vec{\gamma}$ and $\vec{\gamma}^{-1}$ of $\gamma$ in $\hat{\mathscr{L}}_{g, n}^{*}$ are not conjugated in the profinite group $\hat{\Pi}_{g, n}$, it follows that there are at least two such conjugacy classes.

We can now prove Theorem 5.1

For $\alpha, \beta \in \hat{\mathscr{L}}_{g, n}$ such that $\tau_{\alpha}^{k}=\tau_{\beta}^{k}$, by Theorem 5.13, there are lifts $\vec{\alpha}, \vec{\beta} \in \hat{\mathscr{L}}_{g, n}^{*}$ such that the ordered pairs $\left(\tau_{\hat{s}_{1}(\vec{\alpha})}^{k}, \tau_{\hat{s}_{2}(\vec{\alpha})}^{k}\right)$ and $\left(\tau_{\hat{s}_{1}(\vec{\beta})}^{k}, \tau_{\hat{s}_{2}(\vec{\beta})}^{k}\right)$ are in the same $\hat{\Pi}_{g, n^{-}}$ orbit. Since the injective map $\hat{s}_{*}^{k}: \hat{\mathscr{L}}_{g, n}^{*} \hookrightarrow \check{\Gamma}_{g, n+1} \times \check{\Gamma}_{g, n+1}$ is $\hat{\Pi}_{g, n}$-equivariant, it follows that $\vec{\alpha}$ and $\vec{\beta}$ are conjugated in $\hat{\Pi}_{g, n}$, i.e. $\alpha=\beta$.

Remark 5.14. From the identity $\hat{\mathscr{L}}_{g, n}^{*} \cap \Gamma_{g, n+1}=\mathscr{L}_{g, n}^{*}$, it follows that if we denote by $\mathcal{D}^{k}$, for $k \in \hat{\mathbb{Z}}$, the set of $k$-th powers of Dehn twists of the Teichmüller group $\Gamma_{g, n}$ and by $\check{\mathcal{D}}^{k}$ its closure in the procongruence completion $\check{\Gamma}_{g, n}$, the identity $\breve{\mathcal{D}}^{k} \cap \Gamma_{g, n}=\mathcal{D}^{k}$ holds if $k \in \mathbb{Z}$, otherwise this intersection is empty. Together with Theorem 5.1, this implies that, for $\gamma, \gamma^{\prime} \in \hat{\mathscr{L}}$ and $k, k^{\prime} \in \hat{\mathbb{Z}} \backslash\{0\}$, the identity $\tau_{\gamma}^{k}=\tau_{\gamma^{\prime}}^{k^{\prime}}$ holds if and only if $\gamma=\gamma^{\prime}$ and $k=k^{\prime}$.

\section{Centralizers of profinite Dehn twists in $\check{\Gamma}_{g, n}$}

An immediate consequence of Theorem 5.1 and Theorem 4.5 is a description of centralizers of powers of profinite Dehn twists in the procongruence Teichmüller group:

Corollary 6.1. For $2 g-2+n>0$, let $\gamma \in \hat{\mathscr{L}}_{g, n}$ and $k \in \hat{\mathbb{Z}} \backslash 0$. Then, the centralizer in $\check{\Gamma}_{g, n}$ of the element $\tau_{\gamma}^{k}$ coincides with the stabilizer $\check{\Gamma}_{\gamma}$ of the profinite s.c.c. $\gamma$ in $\hat{\mathscr{L}}_{g, n}$.

The center of the procongruence Teichmüller group $\check{\Gamma}_{g, n}$ and the centralizers of its open subgroups are then also determined:

Corollary 6.2. Let $2 g-2+n>0$. For every open subgroup $U$ of $\check{\Gamma}_{g, n}$, it holds that:

$$
Z\left(\check{\Gamma}_{g, n}\right)=Z_{\check{\Gamma}_{g, n}}(U)=Z\left(\Gamma_{g, n}\right)
$$

Thus, all these groups are trivial for $(g, n) \neq(1,1),(2,0)$, otherwise, they are generated by the hyperelliptic involution. In particular, by Theorem 4.5, for $\vec{\gamma}$ an oriented s.c.c. on the Riemann surface $S_{g, n}$, it holds that $Z\left(\check{\Gamma}_{\vec{\gamma}}\right)=Z\left(\Gamma_{\vec{\gamma}}\right)$. 
Proof. For $g \leq 2$, this is just Proposition 3.2 in [7. Let us then proceed by induction on the genus. For $g \geq 3$, let $\gamma$ be an s.c.c. on $S_{g, n}$ bounding an unpunctured genus 1 subsurface of $S_{g, n}$. By Corollary 6.1 for every $k \in \hat{\mathbb{Z}} \backslash 0$, the centralizer of the Dehn twist $\tau_{\gamma}^{k}$ is the stabilizer $\check{\Gamma}_{\gamma}$. By Theorem 4.5. $\check{\Gamma}_{\gamma}$ is the closure of the discrete stabilizer $\Gamma_{\gamma}=\Gamma_{\vec{\gamma}}$. In particular, $\check{\Gamma}_{\gamma}=\check{\Gamma}_{\vec{\gamma}}$ holds. By Theorem 4.5, there is then a short exact sequence:

$$
1 \rightarrow \hat{\mathbb{Z}} \cdot \tau_{\gamma} \rightarrow \check{\Gamma}_{\gamma} \rightarrow \check{\Gamma}_{g_{1}, n_{1}} \times \check{\Gamma}_{1,1} \rightarrow 1
$$

From the induction hypothesis, it then follows that the centralizer $Z_{\check{\Gamma}_{\gamma}}\left(\check{\Gamma}_{\gamma} \cap U\right)$ is the closure in $\check{\Gamma}_{\gamma}$ of the center of the discrete subgroup $\Gamma_{\gamma}$. Hence, the centralizer $Z_{\check{\Gamma}_{\gamma}}\left(\check{\Gamma}_{\gamma} \cap U\right)$ is the closed cyclic subgroup generated by the half Dehn twist $\tau_{\gamma}^{1 / 2}$ about $\gamma$ which restricts to the identity on the subsurface $S_{g_{1}, n_{1}}$.

The open subgroup $U$ of $\check{\Gamma}_{g, n}$ contains a power of $\tau_{\gamma}$. Therefore, there holds $Z_{\check{\Gamma}_{g, n}}(U) \leq \check{\Gamma}_{\gamma}$ and then $Z_{\check{\Gamma}_{g, n}}(U) \leq Z_{\check{\Gamma}_{\gamma}}\left(\check{\Gamma}_{\gamma} \cap U\right)$. So, the centralizer of $U$ in $\check{\Gamma}_{g, n}$ is topologically generated by a power of the half Dehn twist $\tau_{\gamma}^{1 / 2}$. But no non-trivial power of $\tau_{\gamma}^{1 / 2}$ is in the center of $\Gamma_{g, n} \cap U$. Therefore, $Z_{\check{\Gamma}_{g, n}}(U)=\{1\}$ holds.

Let $\gamma$ be an s.c.c. on $S_{g, n}$. It is reasonable to expect that the set of profinite Dehn twists of $\check{\Gamma}_{g, n}$, which are contained in the stabilizer $\check{\Gamma}_{\gamma}$, is the closure of the set of Dehn twists contained in the discrete stabilizer $\Gamma_{\gamma}$. By virtue of Remark 4.7. another way to state this is to say that the profinite Dehn twists of $\check{\Gamma}_{g, n}$, contained in $\check{\Gamma}_{\gamma}$, are parametrized by the vertices of the closed star of $\gamma$ in the complex of profinite curves $L\left(\hat{\Pi}_{g, n}\right)$. More precisely, the following holds:

Theorem 6.3. For $2 g-2+n>0$, let $\alpha, \beta \in \hat{\mathscr{L}}_{g, n}$ and let $\overline{\operatorname{St}}(\beta)$ be the closed star of $\beta$ inside the complex of profinite curves $L\left(\hat{\Pi}_{g, n}\right)$.

i) If $\alpha \in \overline{\operatorname{St}}(\beta)_{0}$, then $\check{\Gamma}_{\beta} \cap \hat{\mathbb{Z}} \cdot \tau_{\alpha}=\hat{\mathbb{Z}} \cdot \tau_{\alpha}$.

ii) If $\alpha \notin \overline{\mathrm{St}}(\beta)_{0}$, then $\check{\Gamma}_{\beta} \cap \hat{\mathbb{Z}} \cdot \tau_{\alpha}=\{1\}$.

Proof. The complex of profinite curves $L\left(\hat{\Pi}_{g, n}\right)$ parametrizes the nerve of the D-M boundary $\partial \widetilde{\mathcal{M}}$ of the profinite covering $\widetilde{\mathcal{M}}$ of $\overline{\mathcal{M}}_{g, n}$ (cf. the proof of Theorem 4.2), while the profinite set of vertices of the $\operatorname{link} \operatorname{Lk}(\beta)$ parametrizes the boundary components of the irreducible component $\tilde{\delta}_{\beta}$ of the boundary $\partial \widetilde{\mathcal{M}}$, associated to the profinite s.c.c. $\beta \in \hat{\mathscr{L}}$, i.e. the irreducible components of the locus where $\tilde{\delta}_{\beta}$ intersects the other irreducible components of $\partial \widetilde{\mathcal{M}}$.

The theorem is then equivalent to the claim that a non-trivial power $\tau_{\alpha}^{h}$ of a profinite Dehn twist of $\check{\Gamma}_{g, n}$, for $\alpha \neq \beta \in \hat{\mathscr{L}}$, stabilizes $\tilde{\delta}_{\beta}$ if and only if the irreducible component $\tilde{\delta}_{\alpha}$, associated to $\alpha$, intersects $\tilde{\delta}_{\beta}$, i.e., if and only if the profinite Dehn twist $\tau_{\alpha}$ generates the inertia group of a boundary component of $\tilde{\delta}_{\beta}$.

The analogous statement is well known when $\tau_{\alpha}$ is a Dehn twist of $\Gamma_{g, n}$ and $\bar{T}_{\beta}$ is the boundary component of the Bers bordification $\bar{T}_{g, n}$ associated to a discrete s.c.c. $\beta$. It can be reformulated by saying that the natural map $\bar{T}_{\beta} \rightarrow \bar{T}_{g, n} /\left\langle\tau_{\alpha}\right\rangle$ is either an embedding or factors through an embedding $\bar{T}_{\beta} /\left\langle\tau_{\alpha}\right\rangle \hookrightarrow \bar{T}_{g, n} /\left\langle\tau_{\alpha}\right\rangle$ and, in the latter case, the subgroup $\left\langle\tau_{\alpha}\right\rangle$ of $\Gamma_{g, n}$ identifies with the inertia group of a boundary component of $\bar{T}_{\beta}$.

Let us explicitly describe the quotient $\widetilde{\mathcal{M}} / \overline{\left\langle\tau_{\alpha}\right\rangle}$. Let $\left\{\Gamma^{\lambda}\right\}_{\lambda \in \Lambda}$ be the tower of all geometric levels of the Teichmüller group $\Gamma_{g, n}$. There is a series of natural 
isomorphisms:

$$
\widetilde{\mathcal{M}}=\lim _{\overleftarrow{\lambda \in \Lambda}} \bar{T}_{g, n} / \Gamma^{\lambda} \cong \lim _{\lambda \in \Lambda}\left[\bar{T}_{g, n} \times\left(\Gamma_{g, n} / \Gamma^{\lambda}\right)\right] / \Gamma_{g, n}=\left(\bar{T}_{g, n} \times \check{\Gamma}_{g, n}\right) / \Gamma_{g, n}
$$

where an element $f \in \Gamma_{g, n}$ acts on the product $\bar{T}_{g, n} \times \check{\Gamma}_{g, n}$ by the formula $f \cdot(x, h)=$ $(f \cdot x, f h)$. Then, there holds:

$$
\widetilde{\mathcal{M}} / \overline{\left\langle\tau_{\alpha}\right\rangle} \cong\left[\bar{T}_{g, n} \times\left(\check{\Gamma}_{g, n} / \overline{\left\langle\tau_{\alpha}\right\rangle}\right)\right] / \Gamma_{g, n}
$$

Let us identify the Bers bordification $\bar{T}_{g, n}$ with its image in the profinite covering $\widetilde{\mathcal{M}}$ of $\overline{\mathcal{M}}_{g, n}$. For every $f \in \check{\Gamma}_{g, n}$, the translation map $\phi_{f}: \bar{T}_{g, n} \hookrightarrow \widetilde{\mathcal{M}}$, defined by the assignment $x \mapsto f \cdot x$, corresponds, via the above isomorphism, to the natural injective map $\bar{T}_{g, n} \times\{f\} \hookrightarrow\left(\bar{T}_{g, n} \times \check{\Gamma}_{g, n}\right) / \Gamma_{g, n}$. In particular, the natural embedding of $\bar{T}_{g, n}$ inside the inverse limit space $\widetilde{\mathcal{M}}$ corresponds to the map $\phi_{1}$. The subspaces $\phi_{f}\left(\bar{T}_{g, n}\right)$ are the analytic leaves of the space $\widetilde{\mathcal{M}}$.

For a given $\beta \in \hat{\mathscr{L}}$, let $\gamma \in \mathscr{L}$ and $h \in \check{\Gamma}_{g, n}$ be such that $h(\gamma)=\beta$. Arguing as above, it follows that the boundary component $\tilde{\delta}_{\beta}$ of $\widetilde{\mathcal{M}}$ is isomorphic to the quotient $\left(\phi_{h}\left(\bar{T}_{\gamma}\right) \times \check{\Gamma}_{\beta}\right) / h \Gamma_{\gamma} h^{-1}$. Then, for $f \in \check{\Gamma}_{\beta}$, the natural injective maps

$$
\bar{T}_{\gamma} \times\{f h\} \hookrightarrow \bar{T}_{g, n} \times \check{\Gamma}_{g, n} / \Gamma_{g, n}
$$

describe the analytic leaves of the boundary component $\tilde{\delta}_{\beta}$ of $\widetilde{\mathcal{M}}$.

Let us define $\psi_{f}$ to be the composition of $\phi_{f}$, for $f \in \check{\Gamma}_{g, n}$, with the quotient map $\widetilde{\mathcal{M}} \rightarrow \widetilde{\mathcal{M}} / \overline{\left\langle\tau_{\alpha}\right\rangle}$. Then, the analytic leaves of $\widetilde{\mathcal{M}} / \overline{\left\langle\tau_{\alpha}\right\rangle}$ are given by the images $\psi_{f}\left(\bar{T}_{g, n}\right)$, for $f \in \bar{\Gamma}_{g, n}$.

In order to describe these leaves, we need to determine the stabilizer of a left coset $f \cdot \overline{\left\langle\tau_{\alpha}\right\rangle}$ in the group $\check{\Gamma}_{g, n}$, for $f \in \check{\Gamma}_{g, n}$, for the action of its discrete subgroup $\Gamma_{g, n}$ by translations.

The stabilizer of the left coset $f \cdot \overline{\left\langle\tau_{\alpha}\right\rangle}$ for the action of the group $\check{\Gamma}_{g, n}$ on itself by translations is clearly the conjugate subgroup $f \overline{\left\langle\tau_{\alpha}\right\rangle} f^{-1}=\overline{\left\langle\tau_{f(\alpha)}\right\rangle}$. So, the stabilizer for the action of $\Gamma_{g, n}$ is the intersection $\Gamma_{g, n} \cap \overline{\left\langle\tau_{f(\alpha)}\right\rangle}$. By Remark 5.14, this intersection equals $\left\langle\tau_{f(\alpha)}\right\rangle$ if $f(\alpha) \in \mathscr{L}$ and is trivial if $f(\alpha) \notin \mathscr{L}$.

The map $\psi_{f}: \bar{T}_{g, n} \rightarrow \widetilde{\mathcal{M}} / \overline{\left\langle\tau_{\alpha}\right\rangle}$ is identified with the natural map

$$
\bar{T}_{g, n} \times\{f\} \rightarrow \bar{T}_{g, n} \times\left(\check{\Gamma}_{g, n} / \overline{\left\langle\tau_{\alpha}\right\rangle}\right) / \Gamma_{g, n} .
$$

Therefore, the map $\psi_{f}$ is injective for $f(\alpha) \notin \mathscr{L}$ and factors through the embedding $\phi_{f}\left(\bar{T}_{g, n}\right) /\left\langle\tau_{f(\alpha)}\right\rangle \hookrightarrow \widetilde{\mathcal{M}} / \overline{\left\langle\tau_{\alpha}\right\rangle}$, for $f(\alpha) \in \mathscr{L}$.

For a given $\beta \in \hat{\mathscr{L}}$, let, as above, $\gamma \in \mathscr{L}$ and $h \in \check{\Gamma}_{g, n}$ be such that $h(\gamma)=\beta$. For $f \in \check{\Gamma}_{\beta}$, the natural map

$$
\bar{T}_{\gamma} \times\{f h\} \rightarrow \bar{T}_{g, n} \times\left(\check{\Gamma}_{g, n} / \overline{\left\langle\tau_{\alpha}\right\rangle}\right) / \Gamma_{g, n}
$$

is then injective unless $f h(\alpha) \in \mathscr{L}$ and the subgroup $\left\langle\tau_{\alpha}\right\rangle$ identifies with the inertia group of a boundary component of the analytic leaf $\bar{T}_{\gamma} \times\{f h\}$ of $\tilde{\delta}_{\beta}$, in which case, the closed cyclic subgroup $\overline{\left\langle\tau_{\alpha}\right\rangle}$ identifies with the inertia group of a boundary component of $\tilde{\delta}_{\beta}$. 
We conclude that the natural map $\tilde{\delta}_{\beta} \rightarrow \widetilde{\mathcal{M}} / \overline{\left\langle\tau_{\alpha}\right\rangle}$ is either injective or factors through an embedding $\tilde{\delta}_{\beta} / \overline{\left\langle\tau_{\alpha}\right\rangle} \hookrightarrow \widetilde{\mathcal{M}} / \overline{\left\langle\tau_{\alpha}\right\rangle}$, where the subgroup $\overline{\left\langle\tau_{\alpha}\right\rangle}$ identifies with the inertia group of a boundary component of $\tilde{\delta}_{\beta}$. This completes the proof of the theorem.

Thanks to the above results, sets of commuting powers of profinite Dehn twists in the procongruence Teichmüller group and their centralizers can be characterized as in the discrete case:

Corollary 6.4. For $2 g-2+n>0$, there holds:

i) A set of non-trivial powers of profinite Dehn twists $\left\{\tau_{\gamma_{0}}^{h_{0}}, \ldots, \tau_{\gamma_{k}}^{h_{k}}\right\}$ of $\check{\Gamma}_{g, n}$ consists of mutually commuting elements if and only if the set of profinite s.c.c.'s $\left\{\gamma_{0}, \ldots, \gamma_{k}\right\}$ is a simplex of $L\left(\hat{\Pi}_{g, n}\right)$.

ii) Let there be given a $k$-simplex $\sigma=\left\{\gamma_{0}, \ldots, \gamma_{k}\right\} \in L\left(\hat{\Pi}_{g, n}\right)$ and $h_{0}, \ldots, h_{k} \in$ $\hat{\mathbb{Z}} \backslash\{0\}$. Then, the centralizer $Z_{\check{\Gamma}_{g, n}}\left(\tau_{\gamma_{0}}^{h_{0}}, \ldots, \tau_{\gamma_{k}}^{h_{k}}\right)$ fits in the exact sequence:

$$
1 \rightarrow Z_{\check{\Gamma}_{g, n}}\left(\tau_{\gamma_{0}}^{h_{0}}, \ldots, \tau_{\gamma_{k}}^{h_{k}}\right) \rightarrow \check{\Gamma}_{\sigma} \rightarrow \Sigma_{\sigma},
$$

where $\check{\Gamma}_{\sigma}$ is the stabilizer of the simplex $\sigma$ for the action of $\check{\Gamma}_{g, n}$ on $L\left(\hat{\Pi}_{g, n}\right)$ and $\Sigma_{\sigma}$ is the group of permutations on the set $\sigma=\left\{\gamma_{0}, \ldots, \gamma_{k}\right\}$.

Proof. The first point follows from Corollary 6.1, Theorem 6.3 and induction on $k$. The second point follows from Corollary 6.1 Theorem 4.5 and induction on $k$.

For $\sigma=\left\{\gamma_{0}, \ldots, \gamma_{k}\right\}$ a $k$-simplex of $L\left(\hat{\Pi}_{g, n}\right)$, let $\mathscr{T}_{\sigma}$ be the closed abelian subgroup of $\check{\Gamma}_{g, n}$ spanned by the profinite Dehn twists $\tau_{\gamma_{0}}, \ldots, \tau_{\gamma_{k}}$. From Corollary 6.4, it follows that:

Corollary 6.5. For $2 g-2+n>0$, let $\sigma=\left\{\gamma_{0}, \ldots, \gamma_{s}\right\}$ and $\sigma^{\prime}=\left\{\delta_{0}, \ldots, \delta_{t}\right\}$ be two simplices of $L\left(\hat{\Pi}_{g, n}\right)$. With the above notation, if the intersection $\mathscr{T}_{\sigma} \cap \mathscr{T}_{\sigma^{\prime}}$ is open in $\mathscr{T}_{\sigma}$, then there holds $\sigma \subseteq \sigma^{\prime}$ and hence $\mathscr{T}_{\sigma} \cap \mathscr{T}_{\sigma^{\prime}}=\mathscr{T}_{\sigma}$.

Proof. By ii) of Corollary 6.4, since $\mathscr{T}_{\sigma} \cap \mathscr{T}_{\sigma^{\prime}}$ is open in $\mathscr{T}_{\sigma}$, the centralizer of the group $\mathscr{T}_{\sigma^{\prime}}$ is contained in that of $\mathscr{T}_{\sigma}$. But then, from i) of Corollary 6.4, it follows that there is an inclusion of simplices $\sigma \subseteq \sigma^{\prime}$.

Let us now compute the normalizer of the closed subgroup of $\check{\Gamma}_{g, n}$ spanned by a set of profinite Dehn twists:

Theorem 6.6. For $2 g-2+n>0$, let there be given $\sigma=\left\{\gamma_{0}, \ldots, \gamma_{k}\right\}$ a simplex of $L\left(\hat{\Pi}_{g, n}\right)$ and $h_{0}, \ldots, h_{k} \in \hat{\mathbb{Z}} \backslash\{0\}$. Then, the normalizer in $\check{\Gamma}_{g, n}$ of the closed subgroup generated by the set of powers of Dehn twists $\left\{\tau_{\gamma_{0}}^{h_{0}}, \ldots, \tau_{\gamma_{k}}^{h_{k}}\right\}$ is the stabilizer $\check{\Gamma}_{\sigma}$ of the simplex $\sigma$ for the action of $\check{\Gamma}_{g, n}$ on the complex of profinite curves $L\left(\hat{\Pi}_{g, n}\right)$.

Proof. An element of $\check{\Gamma}_{\sigma}$ normalizes the closed subgroup generated by $\tau_{\gamma_{0}}^{h_{0}}, \ldots, \tau_{\gamma_{k}}^{h_{k}}$ of $\check{\Gamma}_{g, n}$. Then let $f \in \check{\Gamma}_{g, n}$ be an element which also normalizes this subgroup. For all $0 \leq i \leq k$, the identity $f \tau_{\gamma_{i}}^{h_{i}} f^{-1}=\tau_{f\left(\gamma_{i}\right)}^{h_{i}}=\tau_{\gamma_{0}}^{m_{0}} \ldots \tau_{\gamma_{k}}^{m_{k}}$ holds, for some multindex $\left(m_{0}, \ldots, m_{k}\right) \in(\hat{\mathbb{Z}})^{k+1}$. By the following lemma, it then holds that $f \in \check{\Gamma}_{\sigma}$. 
Lemma 6.7. For $2 g-2+n>0$, let there be given $\gamma \in \hat{\mathscr{L}}, h \in \hat{\mathbb{Z}} \backslash 0,\left\{\gamma_{0}, \ldots, \gamma_{k}\right\} \in$ $L\left(\hat{\Pi}_{g, n}\right)$ a simplex and $h_{0}, \ldots, h_{k} \in \hat{\mathbb{Z}}$. Then, there is an identity $\tau_{\gamma}^{h}=\tau_{\gamma_{0}}^{h_{0}} \ldots \cdot \tau_{\gamma_{k}}^{h_{k}}$ if and only if, for some $0 \leq i \leq k$, it holds that $\gamma_{i}=\gamma, h_{i}=h$ and $h_{j}=0$, for all $j \neq i$.

Proof. It is easy to prove that, since $\left\{\tau_{\gamma_{0}}^{h_{0}}, \ldots, \tau_{\gamma_{k}}^{h_{k}}\right\}$ is a set of commuting elements, if the given identity holds, each of its elements commutes with $\tau_{\gamma}^{h}$. Then, the conclusion of the lemma follows from $i$ ) of Corollary 6.4.

The results of this section also imply that the simplicial profinite complex $L\left(\hat{\Pi}_{g, n}\right)$ parametrizes all the closed abelian subgroups of $\check{\Gamma}_{g, n}$ spanned by powers of profinite Dehn twists. Let us state this more precisely.

The set of closed subgroups of a profinite group is the inverse limit of the inverse system formed by the finite sets of subgroups of its finite discrete quotients. Hence, it is a profinite set. Then let $\check{\mathscr{S}}$ be the profinite set of all closed subgroups of $\check{\Gamma}_{g, n}$. The profinite group $\check{\Gamma}_{g, n}$ acts continuously by conjugation on $\check{\mathscr{S}}$.

A weight function on $\hat{\mathscr{L}}$ is a $\check{\Gamma}_{g, n}$-equivariant function $w: \hat{\mathscr{L}} \rightarrow \mathbb{N}^{+}$, where we let $\check{\Gamma}_{g, n}$ act trivially on $\mathbb{N}^{+}$.

Then let $\iota(w)_{k}: C\left(S_{g, n}\right)_{k} \hookrightarrow \check{\mathscr{S}}$ be the embedding defined by sending a $k$ simplex $\left\{\gamma_{0}, \ldots, \gamma_{k}\right\}$ to the closed subgroup $\hat{\mathbb{Z}} \cdot \tau_{\gamma_{0}}^{w\left(\gamma_{0}\right)} \oplus \ldots \oplus \hat{\mathbb{Z}} \cdot \tau_{\gamma_{k}}^{w\left(\gamma_{k}\right)}$ of $\check{\Gamma}_{g, n}$ and let $\bar{C}^{w}\left(S_{g, n}\right)_{k}$ be the closure, in the profinite topology, of the image of $\iota(w)_{k}$ in $\check{\mathscr{S}}$.

Proposition 6.8. Let $2 g-2+n>0$. For every weight function $w: \hat{\mathscr{L}} \rightarrow \mathbb{N}^{+}$and $0 \leq k \leq 3 g-3+n$, there is a natural $\check{\Gamma}_{g, n}$-equivariant continuous isomorphism:

$$
\Delta_{k}^{w}: L\left(\hat{\Pi}_{g, n}\right)_{k} \stackrel{\sim}{\rightarrow} \bar{C}^{w}\left(S_{g, n}\right)_{k} .
$$

Proof. The map $\Delta_{k}^{w}$ exists by the universal property of the $\check{\Gamma}_{g, n}$-completion. If, for two simplices $\sigma, \sigma^{\prime} \in L\left(\hat{\Pi}_{g, n}\right)_{k}$, it holds that $\Delta_{k}^{w}(\sigma)=\Delta_{k}^{w}\left(\sigma^{\prime}\right)$, the intersection $\mathscr{T}_{\sigma} \cap \mathscr{T}_{\sigma^{\prime}}$ is open in both groups $\mathscr{T}_{\sigma}$ and $\mathscr{T}_{\sigma^{\prime}}$. From Corollary 6.5, it then follows that $\sigma=\sigma^{\prime}$.

By Proposition 6.8, for every weight function $w: \hat{\mathscr{L}} \rightarrow \mathbb{N}^{+}$there is defined a simplicial profinite complex $\bar{C}^{w}\left(S_{g, n}\right)$, endowed with a natural, continuous $\check{\Gamma}_{g, n^{-}}$ action, naturally isomorphic to $L\left(\hat{\Pi}_{g, n}\right)$, which we call the weighted group-theoretic procongruence curve complex. So, the above results sum up in an intrinsic virtual description of the complex of profinite curves $L\left(\hat{\Pi}_{g, n}\right)$.

Theorem 6.9. Let $2 g-2+n>0$. For any given open normal subgroup $\check{\Gamma}^{\lambda}$ of $\check{\Gamma}_{g, n}$, the simplicial profinite complex $L\left(\hat{\Pi}_{g, n}\right)$ is naturally identified with the simplicial complex whose set of $k$-simplices, for $k \geq 0$, consists of primitive free $\hat{\mathbb{Z}}$-modules of rank $k+1$ contained in $\check{\Gamma}^{\lambda}$, generated by powers of profinite Dehn twists.

Remark 6.10. Observe that $L\left(\hat{\Pi}_{g, n}\right)$ is a flag complex, i.e. sets of vertices which are pairwise joinable are joinable. The barycentric subdivision of $L\left(\hat{\Pi}_{g, n}\right)$ is then more simply described as the flag complex associated to the poset of closed abelian subgroups of $\check{\Gamma}_{g, n}$ generated by profinite Dehn twists. 


\section{Faithfulness of Galois representations}

In this section, for $2 g-2+n>0$, we denote by $\mathcal{M}_{g, n}$ the stack of smooth algebraic curves defined over some number field and by $\overline{\mathcal{M}}_{g, n}$ its D-M compactification (in contrast with Sections 2 6] where we considered only complex algebraic curves). Both stacks are defined over $\operatorname{Spec}(\mathbb{Q})$.

Let $C$ be a smooth $n$-punctured, genus $g$ curve, defined over a number field $\mathbb{k}$. To give such a curve, it is equivalent to give a point $\xi: \operatorname{Spec}(\mathbb{k}) \rightarrow \mathcal{M}_{g, n}$. If $\mathscr{C} \rightarrow \mathcal{M}_{g, n}$ denotes the universal $n$-punctured, genus $g$ curve, the curve $C$ is isomorphic to the fiber $\mathscr{C}_{\xi}$. Let $\bar{\xi}$ be the geometric point of $\mathcal{M}_{g, n}$ associated to $\xi$ and a given embedding $\mathbb{k} \subset \overline{\mathbb{Q}}$, let $\tilde{\xi}$ be a closed point of $C \times_{\mathbb{k}} \overline{\mathbb{Q}}$ (which we identify with $\mathscr{C}_{\bar{\xi}}$ ) and let $G_{\mathbb{Q}}$ be the absolute Galois group. There is then a commutative exact diagram:

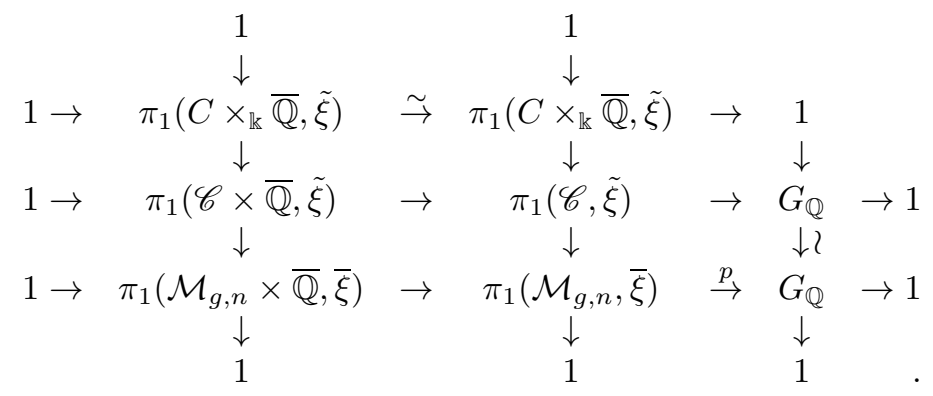

Let $G_{\mathbb{k}}$ be the absolute Galois group of $\mathbb{k}$. Then, the group $G_{\mathbb{k}}$ identifies with an open subgroup of $G_{\mathbb{Q}}$ and the algebraic fundamental group $\pi_{1}\left(\mathcal{M}_{g, n} \times \mathbb{k}, \bar{\xi}\right)$ with the inverse image $p^{-1}\left(G_{\mathbb{k}}\right)$.

Moreover, the point $\xi \in \mathcal{M}_{g, n}$ induces a homomorphism on algebraic fundamental groups $s_{\xi}: G_{\mathbb{k}} \rightarrow \pi_{1}\left(\mathcal{M}_{g, n} \times \mathbb{k}, \bar{\xi}\right)$ which is a canonical section of the natural epimorphism $p: \pi_{1}\left(\mathcal{M}_{g, n} \times \mathbb{k}, \bar{\xi}\right) \rightarrow G_{\mathbb{k}}$.

There are various representations associated to the above diagram. To the middle column is associated the arithmetic universal monodromy representation:

$$
\mu_{g, n}: \pi_{1}\left(\mathcal{M}_{g, n}, \bar{\xi}\right) \rightarrow \operatorname{Out}\left(\pi_{1}\left(C \times_{\mathbb{k}} \overline{\mathbb{Q}}, \tilde{\xi}\right)\right),
$$

which encloses all the others. Composing this representation with the section $s_{\xi}$, we indeed get the outer monodromy representation associated to the curve $C / \mathbb{k}$ :

$$
\rho_{C}: G_{\mathbb{k}} \rightarrow \operatorname{Out}\left(\pi_{1}\left(C \times_{\mathbb{k}} \overline{\mathbb{Q}}, \tilde{\xi}\right)\right) .
$$

If, instead, we compose the arithmetic universal monodromy representation with the natural monomorphism $\pi_{1}\left(\mathcal{M}_{g, n} \times \overline{\mathbb{Q}}, \bar{\xi}\right) \hookrightarrow \pi_{1}\left(\mathcal{M}_{g, n}, \bar{\xi}\right)$, we get the geometric universal monodromy representation

$$
\bar{\mu}_{g, n}: \pi_{1}\left(\mathcal{M}_{g, n} \times \overline{\mathbb{Q}}, \bar{\xi}\right) \rightarrow \operatorname{Out}\left(\pi_{1}\left(C \times_{\mathbb{k}} \overline{\mathbb{Q}}, \tilde{\xi}\right)\right) .
$$

Let us fix an embedding $\overline{\mathbb{Q}} \subset \mathbb{C}$ and let us assume that, with the notation of Section 2, $\bar{\xi}=a$ and $\tilde{\xi}=\tilde{a}$ hold. Then, there are natural isomorphisms $\hat{\Gamma}_{g, n} \cong \pi_{1}\left(\mathcal{M}_{g, n} \times \overline{\mathbb{Q}}, \bar{\xi}\right), \hat{\Gamma}_{g, n+1} \cong \pi_{1}\left(\mathcal{M}_{g, n+1} \times \overline{\mathbb{Q}}, \tilde{\xi}\right)$ and $\hat{\Pi}_{g, n} \cong \pi_{1}\left(C \times_{\mathbb{k}} \overline{\mathbb{Q}}, \tilde{\xi}\right)$, and the geometric universal monodromy representation identifies with the profinite universal monodromy representation $\hat{\rho}_{g, n}$ introduced at the beginning of Section 3 .

In this section, we are going to prove that the representation (7.3) is faithful for all hyperbolic curves $C$, thus reproving a result obtained by Matsumoto [17] in 
the affine case and extended, more recently, by Hoshi and Mochizuki [15] to the projective case.

Moreover, we will show that the representation (7.2) is faithful if and only if the representation (7.4) is faithful, i.e. if the subgroup congruence property holds for $\Gamma_{g, n}$ (this result appeared as well in [15]). In particular, by Theorem 2.10 in [8], it will follow that $\mu_{g, n}$ is faithful for $2 g-2+n>0$ and $g \leq 2$. Let us start by giving the following definition:

Definition 7.1. Let $\Gamma_{g, n}^{\prime}$, for $2 g-2+n>0$, be a profinite completion of the Teichmüller group. We denote by $\operatorname{Aut}^{*}\left(\Gamma_{g, n}^{\prime}\right)$ the group of automorphisms of $\Gamma_{g, n}^{\prime}$ which preserve the set of closed cyclic subgroups of $\Gamma_{g, n}^{\prime}$ generated by profinite Dehn twists. From the definition of profinite Dehn twists, it follows that $\operatorname{Inn}\left(\Gamma_{g, n}^{\prime}\right) \unlhd$ $\operatorname{Aut}^{*}\left(\Gamma_{g, n}^{\prime}\right)$. Then let $\operatorname{Out}^{*}\left(\Gamma_{g, n}^{\prime}\right)$ be the quotient of these two groups.

Let us identify $\hat{\Gamma}_{g, n}$ with the normal subgroup $\pi_{1}\left(\mathcal{M}_{g, n} \times \overline{\mathbb{Q}}, \bar{\xi}\right)$ of $\pi_{1}\left(\mathcal{M}_{g, n}, \bar{\xi}\right)$. Restriction of inner automorphisms then defines the representation:

$$
\hat{\mathscr{G}}_{g, n}: \pi_{1}\left(\mathcal{M}_{g, n}, \bar{\xi}\right) \rightarrow \operatorname{Aut}^{*}\left(\hat{\Gamma}_{g, n}\right) .
$$

For $2 g-2+n>0$, the procongruence Teichmüller group $\check{\Gamma}_{g, n}$ is defined as the image of the geometric universal monodromy representation $\bar{\mu}_{g, n}$. Thus, it is a normal subgroup of $\operatorname{Im} \mu_{g, n}$ and $\pi_{1}\left(\mathcal{M}_{g, n}, \bar{\xi}\right)$ acts on it by composing the representation $\mu_{g, n}$ with the action by conjugation. So, we get the natural representation, compatible with $\hat{\mathscr{G}}_{g, n}$ :

$$
\check{\mathscr{G}}_{g, n}: \pi_{1}\left(\mathcal{M}_{g, n}, \bar{\xi}\right) \rightarrow \operatorname{Aut}^{*}\left(\check{\Gamma}_{g, n}\right) .
$$

We will see that, thanks to the results of Sections 5 and 6 , the representation $\check{\mathscr{G}}_{g, n}$, while containing all the arithmetic information of the representation $\hat{\mathscr{G}}_{g, n}$, is much more treatable. For the moment, let us observe that, by lifting elements of $G_{\mathbb{Q}}$ to $\pi_{1}\left(\mathcal{M}_{g, n}, \bar{\xi}\right)$, we also get natural compatible representations, for $2 g-2+n>0$ :

$$
\hat{b}_{g, n}: G_{\mathbb{Q}} \rightarrow \operatorname{Out}^{*}\left(\hat{\Gamma}_{g, n}\right) \quad \text { and } \quad \check{b}_{g, n}: G_{\mathbb{Q}} \rightarrow \operatorname{Out}^{*}\left(\check{\Gamma}_{g, n}\right) .
$$

By the characterization of the complex of profinite curves $L\left(\hat{\Pi}_{g, n}\right)$ given in Theorem 6.9. there is a natural continuous representation of the profinite group $\operatorname{Aut}^{*}\left(\check{\Gamma}_{g, n}\right)$ into the group $\operatorname{Aut}\left(L\left(\hat{\Pi}_{g, n}\right)\right)$ of continuous automorphisms of $L\left(\hat{\Pi}_{g, n}\right)$. This representation is faithful:

Theorem 7.2. For $2 g-2+n>0$, there is a natural faithful representation:

$$
\operatorname{Aut}^{*}\left(\check{\Gamma}_{g, n}\right) \hookrightarrow \operatorname{Aut}\left(L\left(\hat{\Pi}_{g, n}\right)\right) \text {. }
$$

Proof. Let $\phi: \check{\Gamma}_{g, n} \rightarrow \check{\Gamma}_{g, n}$ be an automorphism such that $\phi\left(\overline{\left\langle\tau_{\gamma}\right\rangle}\right)=\overline{\left\langle\tau_{\gamma}\right\rangle}$ holds, for every profinite Dehn twist $\tau_{\gamma} \in \check{\Gamma}_{g, n}$; i.e., for every $\gamma \in L\left(\hat{\Pi}_{g, n}\right)_{0}$, there holds $\phi\left(\tau_{\gamma}\right)=\tau_{\gamma}^{\alpha_{\gamma}}$, for some $\alpha_{\gamma} \in \hat{\mathbb{Z}}^{*}$. Let us then show that $\phi$ is the identity.

Let $\gamma_{0}$, for $g \geq 1$ (resp. $g=0$ ), be a non-separating s.c.c. (resp. an s.c.c. bounding a disc with two punctures) on $S_{g, n}$. The associated Dehn twists topologically generate $\check{\Gamma}_{g, n}$. So, if we prove that $\phi\left(\tau_{\gamma_{0}}\right)=\tau_{\gamma_{0}}$, it will follow that $\phi$ is the identity.

Then let $\gamma_{1}$, for $g \geq 1$ (resp. $g=0$ ), be an s.c.c. of the same type as $\gamma_{0}$ and which intersects $\gamma_{0}$, geometrically, only once (resp. only twice). The corresponding Dehn twists then satisfy the braid relation $\tau_{\gamma_{0}} \tau_{\gamma_{1}} \tau_{\gamma_{0}}^{-1}=\tau_{\gamma_{1}}^{-1} \tau_{\gamma_{0}} \tau_{\gamma_{1}}$. Applying the automorphism $\phi$, we get the identity:

$$
\tau_{\gamma_{0}}^{\alpha_{\gamma_{0}}} \tau_{\gamma_{1}}^{\alpha_{\gamma_{1}}} \tau_{\gamma_{0}}^{-\alpha_{\gamma_{0}}}=\tau_{\gamma_{1}}^{-\alpha_{\gamma_{1}}} \tau_{\gamma_{0}}^{\alpha_{\gamma_{0}}} \tau_{\gamma_{1}}^{\alpha_{\gamma_{1}}}
$$


or, equivalently,

$$
\tau_{\tau_{\gamma_{0}}^{\alpha_{\gamma_{0}}\left(\gamma_{1}\right)}}^{\alpha_{\gamma_{1}}}=\tau_{\tau_{\gamma_{1}}^{-\alpha \gamma_{1}}\left(\gamma_{0}\right)}^{\alpha_{\gamma_{0}}}
$$

By Remark 5.14, there holds $\alpha_{\gamma_{0}}=\alpha_{\gamma_{1}}$ (let us then call $u$ this element) and $\tau_{\gamma_{0}}^{u}\left(\gamma_{1}\right)=\tau_{\gamma_{1}}^{-u}\left(\gamma_{0}\right)$ in $\hat{\mathscr{L}}$. For suitable lifts $\tilde{\gamma}_{0}$ and $\tilde{\gamma}_{1} \in \mathscr{L}_{g, n}^{*}$ of $\gamma_{0}$ and $\gamma_{1}$, respectively, the loop $\tilde{\gamma}_{1} \cdot \tilde{\gamma}_{0}^{u}$ is then conjugated in $\hat{\Pi}_{g, n}$ either to the loop $\tilde{\gamma}_{0} \cdot \tilde{\gamma}_{1}^{u}$ or to the loop $\tilde{\gamma}_{1}^{-u} \cdot \tilde{\gamma}_{0}^{-1}$. But now, since the classes of $\tilde{\gamma}_{0}$ and $\tilde{\gamma}_{1}$ in the homology group $H_{1}\left(S_{g, n}, \hat{\mathbb{Z}}\right)$ are linearly independent, this is possible only for $u=1$.

For an open normal subgroup $\check{\Gamma}^{\lambda}$ of $\check{\Gamma}_{g, n}$, let us define Aut* $\left.\check{\Gamma}^{\lambda}\right)$ to be the group of automorphisms of $\check{\Gamma}^{\lambda}$ which preserve the set of primitive closed cyclic subgroups generated by powers of profinite Dehn twists.

Corollary 7.3. For $2 g-2+n>0$, let $\check{\Gamma}^{\lambda}$ be a characteristic open subgroup of $\check{\Gamma}_{g, n}$. Then, the natural representation $\operatorname{Aut}^{*}\left(\check{\Gamma}_{g, n}\right) \rightarrow \operatorname{Aut}^{*}\left(\check{\Gamma}^{\lambda}\right)$, induced by the restriction of automorphisms, is faithful.

Proof. By the characterization of $L\left(\hat{\Pi}_{g, n}\right)$ given in Theorem 6.9 this representation factorizes the faithful representation of Theorem 7.2 .

$$
\operatorname{Aut}^{*}\left(\check{\Gamma}_{g, n}\right) \rightarrow \operatorname{Aut}^{*}\left(\check{\Gamma}^{\lambda}\right) \rightarrow \operatorname{Aut}\left(L\left(\hat{\Pi}_{g, n}\right)\right) .
$$

Therefore, it is faithful as well.

The next theorem is a consequence of the description of centralizers of Dehn twists given in Section 6. It is also the key to implement the GrothendieckTeichmüller Lego in higher genus, on which all the following faithfulness results of this section are based.

Theorem 7.4. Let $(g, n)$ and $\left(g^{\prime}, n^{\prime}\right)$ be such that $2 g-2+n>0,2 g^{\prime}-2+n^{\prime}>0$, $g \geq g^{\prime}$ and $3 g-3+n>3 g^{\prime}-3+n^{\prime}$. Then, there is a natural $G_{\mathbb{Q}^{-}}$equivariant homomorphism:

$$
\operatorname{Out}^{*}\left(\check{\Gamma}_{g, n}\right) \rightarrow \operatorname{Out}^{*}\left(\check{\Gamma}_{g^{\prime}, n^{\prime}}\right) .
$$

Proof. By Theorem 7.2 the natural representation $\operatorname{Aut}^{*}\left(\check{\Gamma}_{g, n}\right) \rightarrow \operatorname{Aut}\left(L\left(\hat{\Pi}_{g, n}\right)\right)$ is faithful. A natural guess then is that the action of $\operatorname{Aut}^{*}\left(\check{\Gamma}_{g, n}\right)$ on the complex of profinite curves also preserves the topological type of the simplices. We actually need and will prove here a slightly weaker assertion:

Lemma 7.5. For $2 g-2+n>0$, the action of $\operatorname{Aut}^{*}\left(\check{\Gamma}_{g, n}\right)$ on $\hat{\mathscr{L}}$ preserves the topological type of non-separating profinite s.c.c.'s and of profinite s.c.c.'s bounding a disc with two punctures.

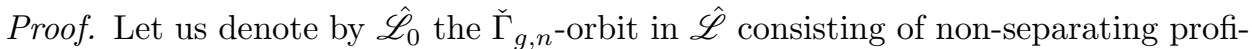
nite s.c.c.'s and, for $n \geq 2$, by $\hat{\mathscr{L}}_{1}$ the $\check{\Gamma}_{g, n}$-orbit in $\hat{\mathscr{L}}$ consisting of profinite s.c.c.'s bounding a disc with two punctures. Let us first show that the action of $\operatorname{Aut}^{*}\left(\check{\Gamma}_{g, n}\right)$ on $\hat{\mathscr{L}}$ preserves the union $\hat{\mathscr{L}}_{0} \cup \hat{\mathscr{L}}_{1}$.

A profinite s.c.c. $\gamma \in \hat{\mathscr{L}}$ is in the $\check{\Gamma}_{g, n}$-orbit of a discrete one and, for all $f \in$ $\operatorname{Aut}^{*}\left(\check{\Gamma}_{g, n}\right)$, there is an isomorphism:

$$
Z_{\check{\Gamma}_{g, n}}\left(\tau_{\gamma}\right) \cong Z_{\check{\Gamma}_{g, n}}\left(\tau_{f(\gamma)}\right)
$$

By Corollary 6.4. any pair of commuting profinite Dehn twists in $Z_{\check{\Gamma}_{g, n}}\left(\tau_{\gamma}\right)$, for $\gamma \in \mathscr{L}$ a discrete s.c.c., is conjugated, inside this group, to a pair of commuting discrete Dehn twists. 
Therefore, the centralizer of a profinite Dehn twist $\tau_{\gamma}$, for $\gamma \in \hat{\mathscr{L}}_{0} \cup \hat{\mathscr{L}}_{1}$, is characterized by the property that, for any pair $\tau_{\alpha}, \tau_{\beta} \in Z_{\check{\Gamma}_{g, n}}\left(\tau_{\gamma}\right)$ of commuting profinite Dehn twists distinct from $\tau_{\gamma}$, there is a profinite Dehn twist $\tau_{\delta}$ in the group $Z_{\check{\Gamma}_{g, n}}\left(\tau_{\gamma}\right)$ which commutes neither with $\tau_{\alpha}$ nor with $\tau_{\beta}$.

The above argument already proves the lemma for $n \leq 1$. Hence, let us assume that $n \geq 2$. We need only to prove that, for any $\gamma \in \hat{\mathscr{L}}_{0}$ and all $f \in \operatorname{Aut}^{*}\left(\check{\Gamma}_{g, n}\right)$, there holds $f(\gamma) \notin \hat{\mathscr{L}}_{1}$.

Let us observe that the set of non-separating profinite Dehn twists forms a single conjugacy class in $\check{\Gamma}_{g, n}$, containing at least two distinct commuting elements for $n \geq 2$. On the other hand, to each pair of punctures on $S_{g, n}$ there corresponds a distinct conjugacy class in $\check{\Gamma}_{g, n}$ of profinite Dehn twists along profinite s.c.c.'s bounding a disc with two punctures, and none of these conjugacy classes contains a pair of distinct commuting elements.

Every automorphism $f$ of $\check{\Gamma}_{g, n}$ sends conjugacy classes to conjugacy classes and pairs of commuting elements to pairs of commuting elements. Therefore, $\gamma \in \hat{\mathscr{L}}_{0}$ implies $f(\gamma) \notin \hat{\mathscr{L}}_{1}$.

In order to prove Theorem 7.4, let us split it into three assertions:

i) for $g \geq 1$, there is a natural homomorphism Out* $\left(\check{\Gamma}_{g, n}\right) \rightarrow \operatorname{Out}^{*}\left(\check{\Gamma}_{g-1, n+2}\right)$;

ii) for $n \geq 2$, there is a natural homomorphism $\operatorname{Out}^{*}\left(\check{\Gamma}_{g, n}\right) \rightarrow \operatorname{Out}^{*}\left(\check{\Gamma}_{g, n-1}\right)$;

iii) for $g \geq 2$, there is a natural homomorphism $\operatorname{Out}^{*}\left(\check{\Gamma}_{g, 1}\right) \rightarrow \operatorname{Out}^{*}\left(\check{\Gamma}_{g}\right)$.

Galois equivariance then follows from the naturality of the above homomorphisms.

In order to prove i), let us choose an orientation for every element of $\hat{\mathscr{L}}$ and let us fix a non-separating s.c.c. $\gamma$ on $S_{g, n}$. By Lemma 7.5. for any given $\tilde{f} \in \operatorname{Aut}^{*}\left(\check{\Gamma}_{g, n}\right)$, there is an $h \in \check{\Gamma}_{g, n}$ such that $\tilde{f}\left(\tau_{\gamma}\right)=\operatorname{inn}(h)\left(\tau_{\gamma}\right)$. Moreover, since for a nonseparating s.c.c. $\gamma$ on $S_{g, n}$ there is a homeomorphism preserving $\gamma$ but switching its orientations, we can choose $h$ such that $h(\vec{\gamma})$ has the fixed orientation.

Therefore, given an $f \in \operatorname{Out}^{*}\left(\check{\Gamma}_{g, n}\right)$, there is a lift $\tilde{f} \in \operatorname{Aut}^{*}\left(\check{\Gamma}_{g, n}\right)$ such that $\tilde{f}\left(\tau_{\gamma}\right)=\tau_{\gamma}$. By Theorem 6.3. the restriction $\tilde{f}_{\gamma}$ of the automorphism $\tilde{f}$ to the subgroup $\check{\Gamma}_{\gamma}=Z_{\breve{\Gamma}_{g, n}}\left(\tau_{\gamma}\right)$ preserves as well the set of closed cyclic groups generated by the profinite Dehn twists of $\check{\Gamma}_{\gamma}$, i.e. the closure of the set of closed cyclic groups generated by the Dehn twists of $\Gamma_{\gamma}$.

From the description of $\check{\Gamma}_{\gamma}$ given in Corollary 6.1, it follows that $\check{\Gamma}_{\vec{\gamma}}$ is the closed normal subgroup of $\check{\Gamma}_{\gamma}$ topologically generated by the Dehn twists contained there. Hence, the automorphism $\tilde{f}_{\gamma}$ can be further restricted to the subgroup $\check{\Gamma}_{\vec{\gamma}}$.

By Theorem 4.5 and Corollary 6.2, there is a natural isomorphism $\check{\Gamma}_{\vec{\gamma}} / Z\left(\check{\Gamma}_{\vec{\gamma}}\right) \cong$ $\check{\Gamma}_{g-1, n+2}$. It then follows that $\tilde{f}_{\gamma}$ induces an automorphism $\bar{f}_{\gamma}$ of $\check{\Gamma}_{g-1, n+2}$.

Let $\tilde{f}^{\prime} \in \operatorname{Aut}^{*}\left(\check{\Gamma}_{g, n}\right)$ be another lift of $f$ with the same properties and let $\bar{f}_{\gamma}^{\prime}$ be the automorphism it induces on $\check{\Gamma}_{g-1, n+2}$. We then have to prove that $\bar{f}_{\gamma}$ and $\bar{f}_{\gamma}^{\prime}$ differ by an inner automorphism of $\check{\Gamma}_{g-1, n+2}$.

By definition, the product $\tilde{f}^{-1} \tilde{f}^{\prime}$ is an inner automorphism $\operatorname{inn}(x)$ of $\check{\Gamma}_{g, n}$, such that $\operatorname{inn}(x)\left(\tau_{\gamma}\right)=\tau_{\gamma}$. By Corollary 6.1, $x$ stabilizes $\gamma$ but then also preserves its chosen orientation. Therefore, $x \in \check{\Gamma}_{\vec{\gamma}}$ holds and the claim follows.

Item ii) is proved with a similar argument to that used for i), where we let $\gamma$ instead be an s.c.c. on $S_{g, n}$ bounding a two-punctured disc. 
Let us now prove item iii). We just need to show that any element of $\operatorname{Aut}^{*}\left(\check{\Gamma}_{g, 1}\right)$ preserves the normal subgroup $\hat{\Pi}_{g}$ of $\check{\Gamma}_{g, 1}$. As a subgroup of $\check{\Gamma}_{g, 1}$, the group $\hat{\Pi}_{g}$ is topologically generated by products of Dehn twists of the form $\tau_{\gamma_{0}} \tau_{\gamma_{1}}^{-1}$, where $\left\{\gamma_{0}, \gamma_{1}\right\}$ is a cut pair on $S_{g, 1}$ consisting of non-separating s.c.c.'s bounding a cylinder containing the puncture. Therefore, it is enough to prove that elements of $\operatorname{Aut}^{*}\left(\check{\Gamma}_{g, 1}\right)$ preserve the topological type of the 1 -simplices of $L\left(\hat{\Pi}_{g, 1}\right)$ corresponding to cut pairs on $S_{g, 1}$ of the above type.

So, given such a cut pair $\left\{\gamma_{0}, \gamma_{1}\right\}$ on $S_{g, 1}$ and any $f \in \operatorname{Aut}^{*}\left(\check{\Gamma}_{g, 1}\right)$, let us show that $\left\{f\left(\gamma_{0}\right), f\left(\gamma_{1}\right)\right\}$ has the same topological type. In order to prove this assertion, we are allowed to modify the given $f$ by an inner automorphism of $\check{\Gamma}_{g, 1}$.

Since $\gamma_{0}$ and $\gamma_{1}$ are non-separating, by Lemma 7.5 and the fact that nonseparating profinite s.c.c.'s form a single conjugacy class in $\check{\Gamma}_{g, 1}$, we may then assume that $f\left(\gamma_{0}\right)=\gamma_{0}$, that $f$ restricts to an automorphism of $\check{\Gamma}_{\vec{\gamma}_{0}}$ and that $f\left(\gamma_{1}\right)$ is non-separating.

The s.c.c. $\gamma_{1}$ determines on $S_{g, 1} \backslash \gamma_{0}$ a separating s.c.c. bounding a disc with two punctures, one of which is the only puncture on $S_{g, 1}$ and the other is one of the two determined by the cutting along $\gamma_{0}$. Then, by virtue of Lemma 7.5 applied to the group $\operatorname{Aut}^{*}\left(\check{\Gamma}_{\vec{\gamma}_{0}}\right)$, the profinite s.c.c. $f\left(\gamma_{1}\right)$ also bounds a disc containing two punctures on the cut surface $S_{g, 1} \backslash \gamma_{0}$. Now, one of these punctures must be the one coming from $S_{g, 1}$, otherwise $f\left(\gamma_{1}\right)$ would bound a genus 1 subsurface on $S_{g, 1}$. Therefore, $\left\{f\left(\gamma_{0}\right), f\left(\gamma_{1}\right)\right\}$ is a cut pair of the same topological type as $\left\{\gamma_{0}, \gamma_{1}\right\}$.

Corollary 7.6. For $2 g-2+n>0$ and $3 g-3+n>0$, the natural representation $\check{b}_{g, n}: G_{\mathbb{Q}} \rightarrow$ Out $^{*}\left(\check{\Gamma}_{g, n}\right)$ is faithful. It follows, in particular, that the representation $\hat{b}_{g, n}: G_{\mathbb{Q}} \rightarrow \operatorname{Out}^{*}\left(\hat{\Gamma}_{g, n}\right)$ is faithful as well.

Proof. For $(g, n)=(0,4),(1,1)$ the above statement is well known and a direct consequence of Belyî's Theorem (cf. [4]). The general case then follows, since, by Theorem 7.4, for $3 g-3+n>1$, there is a natural $G_{\mathbb{Q}^{-}}$equivariant homomorphism $\operatorname{Out}^{*}\left(\check{\Gamma}_{g, n}\right) \rightarrow \operatorname{Out}^{*}\left(\check{\Gamma}_{0,4}\right)$.

As an almost immediate consequence, we get the most important faithfulness result of this section:

Theorem 7.7. Let $C$ be a hyperbolic curve defined over a number field $\mathbb{k}$. Then, the associated outer Galois representation $\rho_{C}: G_{\mathbb{k}} \rightarrow \operatorname{Out}\left(\pi_{1}\left(C \times_{\mathbb{k}} \overline{\mathbb{Q}}, \tilde{\xi}\right)\right)$ is faithful.

Proof. Let us assume that $C$ is the curve which appears in diagram (7.1). From Corollary 7.6, it follows that the homomorphism $\check{\mathscr{G}}_{g, n} \circ s_{\xi}: G_{\mathbb{k}} \rightarrow \operatorname{Aut}^{*}\left(\check{\Gamma}_{g, n}\right)$ is injective. This homomorphism can be described as follows. For $h \in G_{\mathbb{k}}$, the automorphism $\check{\mathscr{G}}_{g, n} \circ s_{\xi}(h)$ of $\check{\Gamma}_{g, n}<\operatorname{Out}\left(\pi_{1}\left(C \times_{\mathbb{k}} \overline{\mathbb{Q}}\right)\right)$ is defined by the assignment, for $f \in \check{\Gamma}_{g, n}$ :

$$
f \longmapsto \rho_{C}(h) \cdot f \cdot \rho_{C}(h)^{-1} \in \operatorname{Out}\left(\pi_{1}\left(C \times_{\mathbb{k}} \overline{\mathbb{Q}}\right)\right) .
$$

Therefore, it follows that $\operatorname{ker} \rho_{C} \leq \operatorname{ker}\left(\check{\mathscr{G}}_{g, n} \circ s_{\xi}\right)=\{1\}$.

Remark 7.8. For suitable choices of tangential base point $\xi$, for instance, if $\mathscr{C}_{\bar{\xi}}$ is a nodal curve with an elliptic tail defined over $\mathbb{Q}$, it is not difficult to infer directly from Belyı̌'s Theorem that the representation $\check{\mathscr{G}}_{g, n} \circ s_{\xi}: G_{\mathbb{Q}} \rightarrow \operatorname{Aut}{ }^{*}\left(\check{\Gamma}_{g, n}\right)$ is faithful. However, this does not imply, at least not formally, the faithfulness of the representation $\check{\mathscr{G}}_{g, n} \circ s_{\xi}$ for any other choice of base point $\xi$. 
A $\mathbb{Q}$-rational point of the moduli stack $\overline{\mathcal{M}}_{g, n}$, given for instance by a suitable graph of punctured projective lines, determines a $\mathbb{Q}$-rational tangential base point of $\mathcal{M}_{g, n}$ and thus a $\pi_{1}\left(\mathcal{M}_{g, n} \times \overline{\mathbb{Q}}, \bar{\xi}\right)$-conjugacy class of splittings of the short exact sequence:

$$
1 \rightarrow \pi_{1}\left(\mathcal{M}_{g, n} \times \overline{\mathbb{Q}}, \bar{\xi}\right) \rightarrow \pi_{1}\left(\mathcal{M}_{g, n}, \bar{\xi}\right) \stackrel{p}{\rightarrow} G_{\mathbb{Q}} \rightarrow 1
$$

Let $\sigma: G_{\mathbb{Q}} \rightarrow \pi_{1}\left(\mathcal{M}_{g, n}, \bar{\xi}\right)$ be a section of $p$ corresponding to one of these splittings. It determines an isomorphism:

$$
\pi_{1}\left(\mathcal{M}_{g, n}, \bar{\xi}\right) \cong \hat{\Gamma}_{g, n} \rtimes_{\tilde{b}_{g, n}} G_{\mathbb{Q}},
$$

where we have identified $\pi_{1}\left(\mathcal{M}_{g, n} \times \overline{\mathbb{Q}}, \bar{\xi}\right)$ with $\hat{\Gamma}_{g, n}$ and $G_{\mathbb{Q}}$ acts on it by means of the representation $\tilde{b}_{g, n}:=\hat{\mathscr{G}}_{g, n} \circ \sigma: G_{\mathbb{Q}} \rightarrow \operatorname{Aut}^{*}\left(\hat{\Gamma}_{g, n}\right)$.

In terms of this isomorphism, the representation $\hat{\mathscr{G}}_{g, n}: \pi_{1}\left(\mathcal{M}_{g, n}, \bar{\xi}\right) \rightarrow \operatorname{Aut}^{*}\left(\hat{\Gamma}_{g, n}\right)$ is then described by sending an element $(f, h) \in \hat{\Gamma}_{g, n} \rtimes_{\sigma} G_{\mathbb{Q}}$ to the automorphism of $\hat{\Gamma}_{g, n}$ given by the composition inn $f \cdot \tilde{b}_{g, n}(h)$.

Proposition 7.9. $\quad$ i) For $2 g-2+n>0$, the kernel of the representation $\hat{\mathscr{G}}_{g, n}: \pi_{1}\left(\mathcal{M}_{g, n}, \bar{\xi}\right) \rightarrow \operatorname{Aut}^{*}\left(\hat{\Gamma}_{g, n}\right)$ can be identified with the center of $\hat{\Gamma}_{g, n}$.

In particular, for $g \leq 2$, the representation $\hat{\mathscr{G}}_{g, n}$ is faithful for $(g, n) \neq$ $(1,1),(2,0)$. Otherwise its kernel is spanned by the hyperelliptic involution.

ii) For $g \geq 3$ and $n \geq 0$, the kernel of the representation $\check{\mathscr{G}}_{g, n}: \pi_{1}\left(\mathcal{M}_{g, n}, \bar{\xi}\right) \rightarrow$ $\operatorname{Aut}^{*}\left(\check{\Gamma}_{g, n}\right)$ can be identified with the congruence kernel of $\hat{\Gamma}_{g, n}$.

Proof. From Corollary 7.6, it follows that $\tilde{b}_{g, n}$ is faithful and also that $\operatorname{Inn}\left(\hat{\Gamma}_{g, n}\right) \cap$ $\operatorname{Im} \tilde{b}_{g, n}=\{1\}$ holds. These two facts yield the first statement of $\left.i\right)$.

By Proposition 3.2 in [8], for $g \leq 2$, there holds $Z\left(\hat{\Gamma}_{g, n}\right)=Z\left(\Gamma_{g, n}\right)$, and this yields the second statement of $i$ ).

As to $i i$ ), it follows from the fact that, by definition, the kernel of the natural homomorphism $\hat{\Gamma}_{g, n} \rightarrow \check{\Gamma}_{g, n}$ is the congruence kernel, by Corollary 6.2, for $g \geq 3$, the natural representation inn: $\check{\Gamma}_{g, n} \rightarrow$ Aut* $\left(\check{\Gamma}_{g, n}\right)$ is faithful and, by Corollary 7.6 , the composition $\tilde{b}_{g, n}^{\prime}:=\check{\mathscr{G}}_{g, n} \circ \sigma$ is faithful and $\operatorname{Inn}\left(\check{\Gamma}_{g, n}\right) \cap \operatorname{Im} \tilde{b}_{g, n}^{\prime}=\{1\}$ holds.

Since the congruence subgroup property holds in genus $\leq 2$ (cf. [3], 7], 8]), from the same argument used in the proof of Theorem 7.7, there then follows:

Corollary 7.10. Let $2 g-2+n>0$. The arithmetic universal monodromy representation $\mu_{g, n}: \pi_{1}\left(\mathcal{M}_{g, n}, \bar{\xi}\right) \rightarrow \operatorname{Out}\left(\pi_{1}\left(C \times_{\mathbb{k}} \overline{\mathbb{Q}}, \tilde{\xi}\right)\right)$ is faithful for $g \leq 2$. Otherwise its kernel can be identified with the congruence kernel of the profinite Teichmüller group $\hat{\Gamma}_{g, n}$.

\section{ACKNOWLEDGEMENTS}

This work began during the author's stay at the University of Costa Rica in San José and completed during his stay at the Department of Mathematics of the University of los Andes in Bogotá. The author thanks both institutions for their support. He also thanks Shinichi Mochizuki who commented on a previous version of this paper and suggested the statement of Lemma 5.11. 


\section{REFERENCES}

[1] William Abikoff, The real analytic theory of Teichmüller space, Lecture Notes in Mathematics, vol. 820, Springer, Berlin, 1980. MR.590044 (82a:32028)

[2] Enrico Arbarello, Maurizio Cornalba, and Pillip A. Griffiths, Geometry of algebraic curves. Volume II, Grundlehren der Mathematischen Wissenschaften [Fundamental Principles of Mathematical Sciences], vol. 268, Springer, Heidelberg, 2011. With a contribution by Joseph Daniel Harris. MR2807457 (2012e:14059)

[3] Mamoru Asada, The faithfulness of the monodromy representations associated with certain families of algebraic curves, J. Pure Appl. Algebra 159 (2001), no. 2-3, 123-147, DOI 10.1016/S0022-4049(00)00056-6. MR:1828935 (2002c:14040)

[4] G. V. Belyı̆, Galois extensions of a maximal cyclotomic field (Russian), Izv. Akad. Nauk SSSR Ser. Mat. 43 (1979), no. 2, 267-276, 479. MR.534593 (80f:12008)

[5] Joan S. Birman, The algebraic structure of surface mapping class groups, Discrete groups and automorphic functions (Proc. Conf., Cambridge, 1975), Academic Press, London, 1977, pp. 163-198. MR0488019(58 \#7596)

[6] Marco Boggi, Profinite Teichmüller theory, Math. Nachr. 279 (2006), no. 9-10, 953-987, DOI 10.1002/mana.200510405. MR2242960 (2008f:14037)

[7] Marco Boggi, The congruence subgroup property for the hyperelliptic modular group: the open surface case, Hiroshima Math. J. 39 (2009), no. 3, 351-362. MR.2569009 (2011a:14049)

[8] M. Boggi, The congruence subgroup property for the hyperelliptic Teichmüller modular group. arXiv:0803.3841v3 (2011).

[9] M. Boggi, Galois covers of moduli spaces of curves and loci of curves with symmetries. arXiv:1111.2372v3 (2012).

[10] Jean-Luc Brylinski, Propriétés de ramification à l'infini du groupe modulaire de Teichmüller (French), Ann. Sci. École Norm. Sup. (4) 12 (1979), no. 3, 295-333. With an appendix in English by Ken Baclawski. MR.559345 (81f:14014)

[11] D. J. Collins, R. I. Grigorchuk, P. F. Kurchanov, and H. Zieschang, Combinatorial group theory and applications to geometry, Springer-Verlag, Berlin, 1998. Translated from the 1990 Russian original by P. M. Cohn; reprint of the original English edition from the series Encyclopaedia of Mathematical Sciences [Algebra. VII, Encyclopaedia Math. Sci., 58, Springer, Berlin, 1993; MR1265269 (95g:57004)]. MR1658468 (2000k:57005)

[12] Edna K. Grossman, On the residual finiteness of certain mapping class groups, J. London Math. Soc. (2) 9 (1974/75), 160-164. MR0405423 (53 \#9216)

[13] Alexandre Grothendieck, Esquisse d'un programme (French, with French summary), Geometric Galois actions, 1, London Math. Soc. Lecture Note Ser., vol. 242, Cambridge Univ. Press, Cambridge, 1997, pp. 5-48. With an English translation on pp. 243-283. MR1483107 (99c:14034)

[14] Yuichiro Hoshi, The exactness of the log homotopy sequence, Hiroshima Math. J. 39 (2009), no. 1, 61-121. MR2499198 (2010b:14035)

[15] Y. Hoshi, S. Mochizuki, On the combinatorial anabelian geometry of nodally nondegenerate outer representations, Hiroshima Math. J. 41 (2011), no. 3, 275-342. MR2895284

[16] Eduard Looijenga, Smooth Deligne-Mumford compactifications by means of Prym level structures, J. Algebraic Geom. 3 (1994), no. 2, 283-293. MR1257324 (94m:14029)

[17] Makoto Matsumoto, Galois representations on profinite braid groups on curves, J. Reine Angew. Math. 474 (1996), 169-219, DOI 10.1515/crll.1996.474.169. MR1390695(97h:14043)

[18] B. Noohi, Fundamental groups of algebraic stacks, J. Inst. Math. Jussieu 3 (2004), no. 1, 69-103, DOI 10.1017/S1474748004000039. MR2036598 (2004k:14003)

[19] B. Noohi, Foundations of topological stacks I. ArXiv: math.AG/0503247 (2005).

[20] P. F. Stebe, Conjugacy separability of certain Fuchsian groups, Trans. Amer. Math. Soc. 163 (1972), 173-188. MR0292949 (45 \#2030)

[21] Ravi Vakil and Kirsten Wickelgren, Universal covering spaces and fundamental groups in algebraic geometry as schemes (English, with English and French summaries), J. Théor. Nombres Bordeaux 23 (2011), no. 2, 489-526. MR.2817942 (2012h:14049)

Departamento de Matemáticas, Universidad de los Andes, Carrera $1^{a} \mathrm{~N}^{o} 18 \mathrm{~A}-10$, Bogotá, Colombia

E-mail address: marco.boggi@gmail.com 